\title{
FABRICATION OF SMART TITANIUM IMPLANTS BY FEMTOSECOND LASER SYNTHESIS
}

by

Sivaprasad Chinnakkannu Vijayakumar

Bachelor of Engineering, Anna University, 2011

\author{
A thesis presented to Ryerson University \\ in partial fulfillment of the requirements for the degree of \\ Master of Applied Science \\ in the program of \\ Mechanical and Industrial Engineering
}

Toronto, Ontario, Canada, 2014

(C) Sivaprasad Chinnakkannu Vijayakumar 2014 


\begin{abstract}
AUTHOR'S DECLARATION
I hereby declare that I am the sole author of this thesis. This is a true copy of the thesis, including any required final revisions, as accepted by my examiners.

I authorize Ryerson University to lend this thesis to other institutions or individuals for the purpose of scholarly research

I further authorize Ryerson University to reproduce this thesis by photocopying or by other means, in total or in part, at the request of other institutions or individuals for the purpose of scholarly research.
\end{abstract}

I understand that my thesis may be made electronically available to the public. 


\title{
FABRICATION OF SMART TITANIUM IMPLANTS BY FEMTOSECOND LASER SYNTHESIS
}

\author{
Sivaprasad Chinnakkannu Vijayakumar
}

Master of Applied Science - 2014

Department of Mechanical \& Industrial Engineering

Ryerson University

\begin{abstract}
A 3-D nanostructure particle network of $\mathrm{TiO}_{2}, \mathrm{TiO}, \mathrm{Ti}_{3} \mathrm{O}$ oxide nanoparticles is synthesized by ultra-short pulsed femtosecond laser irradiation from a Grade 2 pure titanium substrate. This study investigated the properties of the resulting nanostructure and underneath phase transformed surface for biomaterial applications. Controlled tuning of surface chemistry and phases of the 3-D network were found to directly influence the cell mobility. The presented findings support a previously unrealized capacity by nano-core shell like particles and its phases for reducing cell proliferation on a biomaterial. Both osteoblast and fibroblast cells improved controllability and anisotropic migration with the developed nanostructure. The corresponding oxide phases which influenced this controllability was analysed in detail with possible potential in health care industry. The results suggest an effective means to improve biomaterial life thereby increasing implant life.
\end{abstract}




\section{ACKNOWLEDGEMENTS}

I would like to thank my research supervisors Dr. Krishnan Venkatakrishnan and Dr. Bo Tan for their immense support in guiding my research. A special thanks to both my supervisors for their insightful feedback on completion of this thesis and leading me on the right path.

I would like to thank my fellow lab members especially Ms. Priyatha Premnath for teaching me cell culturing techniques and Jasjeet Singh for aiding in illustrations.

Most importantly I would like to thank my parents for their support, prayers and love. 


\section{TABLE OF CONTENTS}

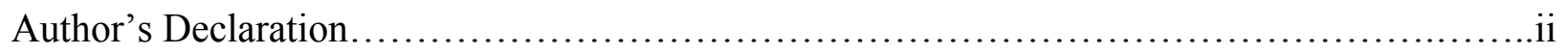

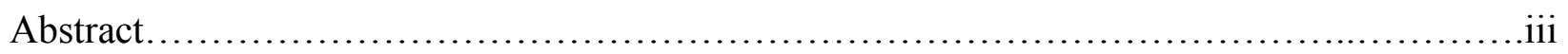

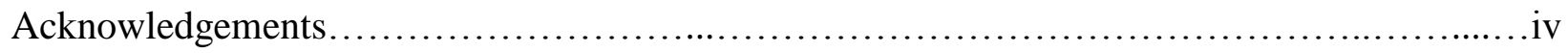

List of Figures..................................................................

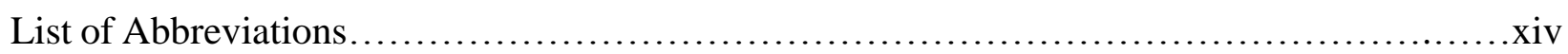

\section{Introduction}

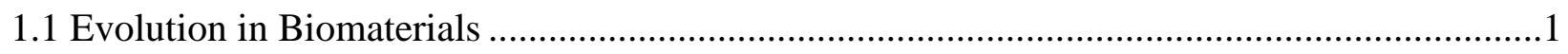

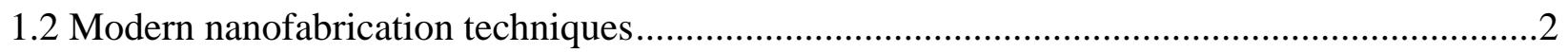

1.2.1 Electro spinning techniques............................................................................ 3

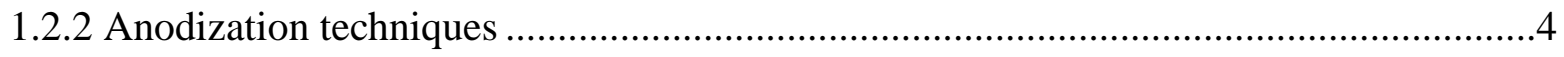

1.3 Functionalization of implant surface and cell interaction ...........................................6

1.4 Concerns over other modern fabrication methods ....................................................

1.5 Previous studies on laser synthesis of titanium............................................................

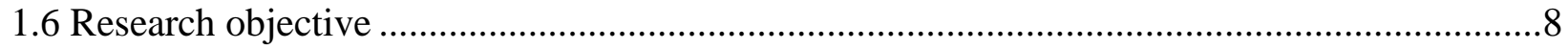

\section{Laser Material Interactions}

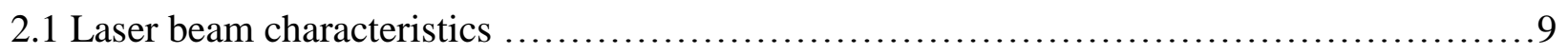

2.1.1 Laser ablation............................................................

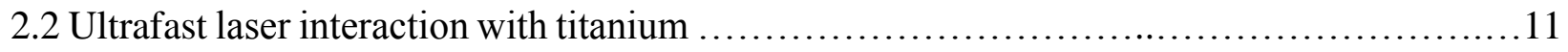

2.2.1 Mechanism of agglomerated nanoparticle network formation during femtosecond laser

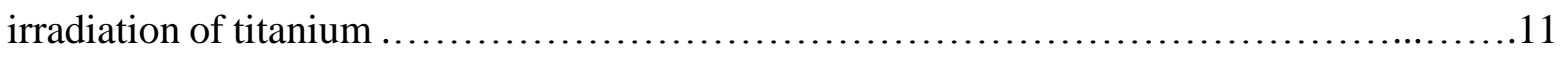

\section{Experimental procedures}

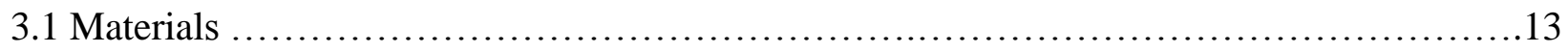

3.2 Laser experimentation..................................................... 13 


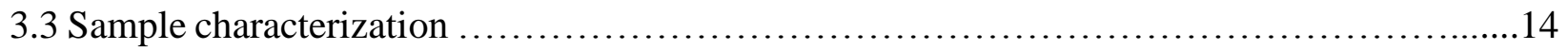

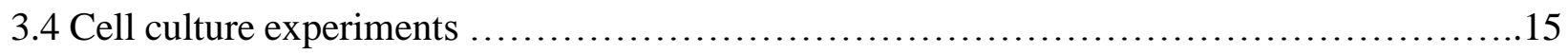

3.4.1 Cell culture-MC3T3-E1 osteoblast cell..........................................15

3.4.2 Cell culture-NIH3T3 fibroblast cell............................................. 15

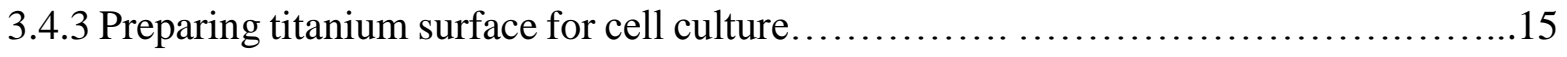

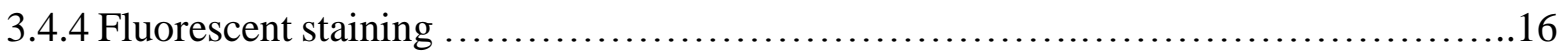

4. Novel Phase/ Non-stoichiometric oxide phase for cell controllability ...................17

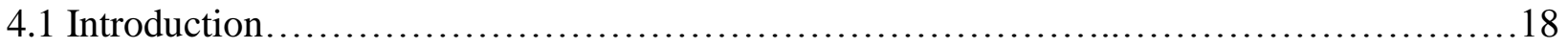

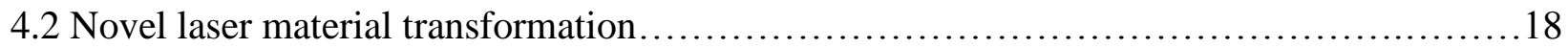

4.3 Phase analysis in engineering novel polymorphic phase oxides ...........................21

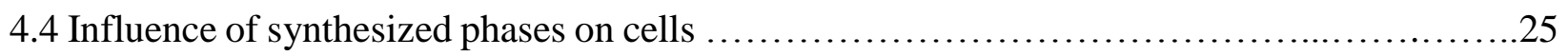

4.4.1 Influence of osteoblast cells on functionalized phases ..............................25

4.4.2 Directional cell mobilization with dominant phase surface .........................27

4.4.3 Single cell channeling by synthesized phase surfaces..............................30

4.4.3.1 Specific cell interactions with rutile dominant surface........................33

4.4.4 Functionalized surface acting as cues for cell repellence..............................34

4.4.5 Influence of fibroblast cells on functionalized phases ............................... 35

4.4.6 Influence of fibroblast cells on hongquiite dominant phase ...........................36

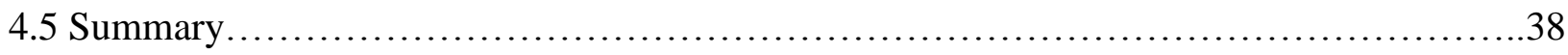

5. Non catalytic/ enzymatic approach of nano-core like structure and cell sustainability

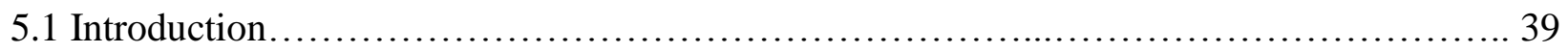

5.2 Generating mechanism of engineered titanium nanostructure ............................ 39

5.3 Elemental composition of the novel nanostructure synthesized ......................... 41

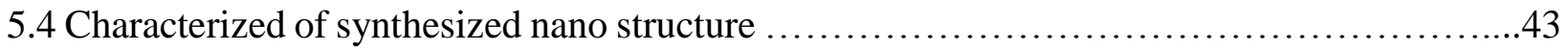


5.5 Oxide phase composition of the synthesized nanostructure $\ldots \ldots \ldots \ldots \ldots \ldots \ldots \ldots \ldots \ldots \ldots . \ldots . \ldots \ldots$

5.6 Characteristic of engineered nanoparticles in the nanofibrous structure....................46

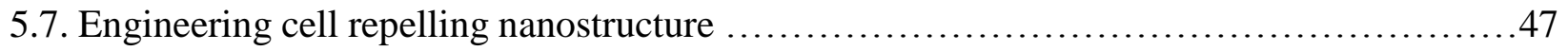

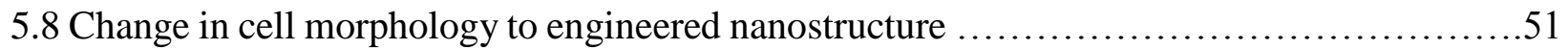

5.8.1 Microvilli manipulation in cells by phase dominant nanostructure.................52

5.8.2 Influence of cell nucleus by phase dominant nanostructure $\ldots \ldots \ldots \ldots \ldots \ldots \ldots \ldots \ldots \ldots . .53$

5.8.3 Influence of cell motility on synthesized nanostructure .........................54

5.8.4 Cell trapping by phase dominant nanostructure on fibroblast cells...................55

5.8.5 Fibroblast cell proliferation on nanostructure density..........................56

5.8.6 Filopodial sensing by fibroblast cell on synthesized nanostructure ..................58

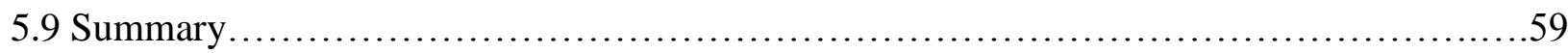

\section{Phase transformed surface and nano printed structure for cell manipulation}

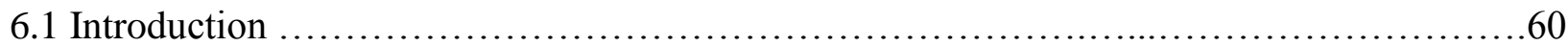

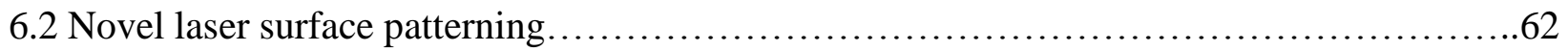

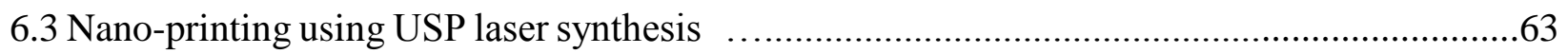

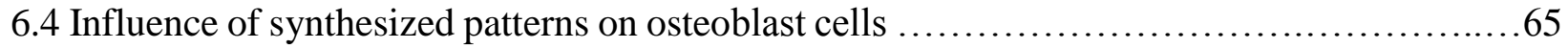

6.5 Influence of selective nano printing on osteoblast cell................................67

6.6 Influence of synthesized pattern on fibroblast cell ...................................69

6.7 Influence of selective nano printing on fibroblast cells............................. 70

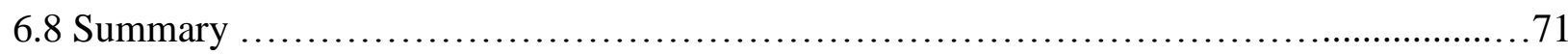

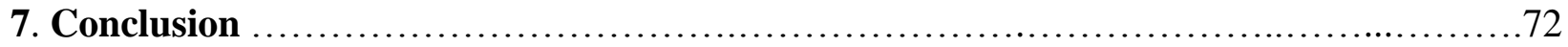

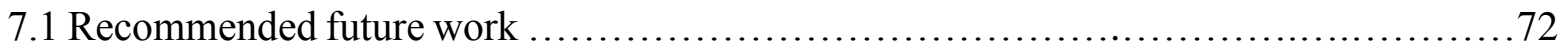

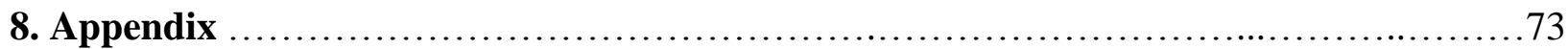

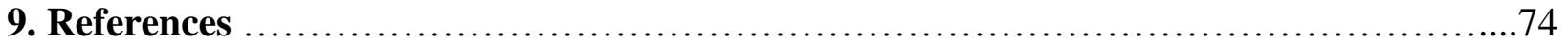




\section{LIST OF FIGURES}

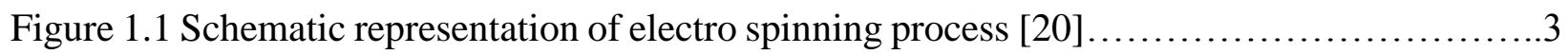

Figure 1.2 FE-SEM of electrospun polymer mixed titanium dioxide powder at a) $10 \mathrm{wt} \%$ b) 20 wt $\%$ c) $30 \mathrm{wt} \%$ with inserts showing higher magnification detail of individual fibers, d) e) and f) showing other surface patterns created using electro spinning $[23,24] \ldots \ldots \ldots \ldots \ldots \ldots \ldots \ldots \ldots . \ldots . \ldots \ldots$

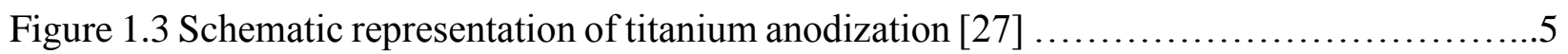

Figure 1.4 SEM micrographs of self-aligned $\mathrm{TiO}_{2}$ nanotubes with different diameter. The micrographs show ordered nanotubes with four different pore sizes between 30 to 100nm [30]

Figure 3.1 Schematic drawing of the experimental setup ................................. 14

Figure 4-1 (A)-(D) Illustrates progression of SEM/EDX line scan of laser transformed titanium surface from $25 \mathrm{MHz}$ ( High laser pulse frequency) to $4 \mathrm{MHz}$ ( Low laser pulse frequency) at constant pulse to pulse separation time ( pulse width-214 Fs ). A constant pulse per unit area of $10 \mathrm{~mm} / \mathrm{s}$ (scanning speed) is maintained. The green pulses in (A-D) represent the intensity or peak power generated per pulse at the above laser condition

Figure 4-2 Shows extent of material transformation with varying laser pulse to pulse separation time at corresponding laser pulse frequency rates $(4,8,12 \& 25 \mathrm{MHz})$. A trend in transformation width with laser pulse repetition rate was also observed

Figure 4-3(A-E) AFM Scans of material transformed surface with native titanium substrate. Figure $3 \mathrm{E}$ is native titanium surface with topographic or phase activity. Figure 3(A-B) height and phase scan data at high laser pulse frequency rate $(25 \mathrm{MHz})$ and figure $3(\mathrm{C}-\mathrm{D})$ are height and phase scans

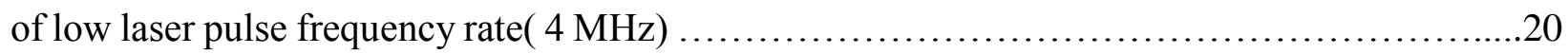

Figure 4-4A represents XRD spectrum of unmodified native $\alpha$-titanium substrate and 4B optical

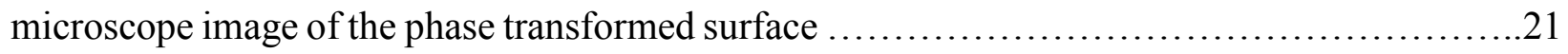

Figure 4-4 (C-D) represents XRD spectrum of titanium oxides phases synthesized on titanium substrate at high $(25 \mathrm{MHz})$ and low $(4 \mathrm{MHz})$ laser pulse frequency $\ldots \ldots \ldots \ldots \ldots \ldots \ldots \ldots \ldots \ldots . . . \ldots \ldots$

Figure 4-5 Graph representing magnitude of TiO oxide synthesis with cumulative pulses........24 
Figure 4-6: SEM images of MC 3T3 osteoblasts on native titanium and phase transformed surface after 24 hours. On the native titanium reference surface cells are flat and attach with the whole body whereas Figure $(C \& E)$ on phase transformed surface cells adhere exclusively on top of the laser irradiated surface .25

Figure 4-7 Adhesion of MC3T3-E1 Osteoblast cells on both phase dominant surfaces in relation to planar reference titanium 26

Figure 4-8 MC3T3-E1 Osteoblast cell activity for 48 hours incubation period on both hongquiite and rutile phase dominant surfaces at two pulse to pulse separation time(214-1428 fs)

Figure 4-9(A-E) MC 3T3 Osteoblast cell activity for 24-48 hours incubation period on both hongquiite (B-C) and rutile dominant surface (D-E) compared to native titanium reference surface .28

Figure 4-10: Illustration of reduction in cell proliferation and single cell channelling by respective dominant phase surfaces synthesized .29

Figure 4-11 (A-D) Single cell channelling on rutile dominant surface with fluorescence images of a single channelled by the rutile dominant phase surface after 48 hours .30

Figure 4-12: Illustrates effect of elongation/ cell stretching in osteoblast cell proliferation on different phase dominant surfaces for 48 hours with varying pulse to pulse separation time .31

Figure 4-13: Graph showing variance in area of cell proliferation with roundness of nuclei. 32

Figure4-14: MC3T3-E1 Osteoblast cell activity for 48 hours at rutile dominant material. A graphic illustration various parts of cell on surface functionalized substrate is represented. 
Figure 4-15 Show preferential growth of cells based on phase dominant material. Figure $15 \mathrm{C}$ cell on native titanium surface Fig 4-15 (A,B,D\&E) are magnified fluorescence data showing the preferential growth alignment with filopodial activity near the oxide phases and cell repellence on

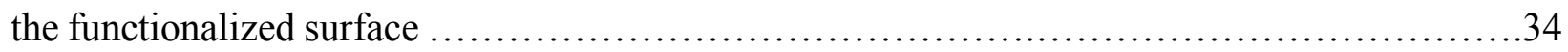

Figure 4-16 Illustration of cell proliferation on both (A,B)hongquiite dominant and (C,D) rutile

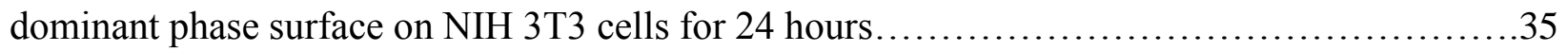

Figure 4-17 (A-E) SEM micrographs of NIH-3T3 fibroblast cell for 24 hours. Fig 17 F fluorescent microscope data of the hongquiite phase dominant surface for 24 hours incubation .36

Figure 4-18 A-E: SEM analysis / Fluorescence microscope data of NIH-3T3 fibroblast cell for 48 hours on both hongquiite and rutile dominant phase surface .... .37

Figure 5-1 Illustration of engineering nanostructure by vapor condensation mechanism on titanium by USP laser. Representative graphical representation of nanostructure density with SEM magnification. i) Appearance of the synthesized nanostructure at high density site; ii) organization of self-assembled highly inter connected nanofibrous structure iii) strands containing individual nanoparticle assembled to form a core shell like nanostructure (A-C) respective nanoparticle size

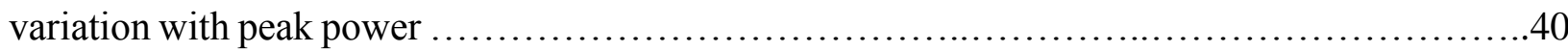

Figure 5-2 Show synthesized nanostructure variation with laser peak power and laser pulse repetition rate (B) Effect of increasing peak power on core shell like nanostructure formation (B1B2) TEM/EDX intensity data indicating variation of titanium to oxygen ratios at low and high laser peak power conditions

Figure 5.3 (A-C) AFM Scans of nanostructured titanium substrate. Figure 3B phase activity on the nanostructure .Figure $3 \mathrm{~A}$ height scan data at high laser pulse frequency rate $(25 \mathrm{MHz})$ and figure $3 \mathrm{C}$ is height scans at low laser pulse frequency rate $(4 \mathrm{MHz})$............................43

Figure 5-4 XRD Micrographs illustrates varying laser peak power on material interactions for synthesis of titanium core shell like nanostructures. A constant pulse width of (214 fs) and (1428fs) was maintained to measure change in intensity of oxide phases synthesized....................44 
Figure 5-5 TEM image and particle size variation of synthesized anatase phase dominant structure

Figure 5-6 TEM image and particle size variation of synthesized Rutile phase dominant structure

Figure 5-7 Graphical Illustration of MC3T3-E1 Osteoblast on native (A) and with nanostructure density having Anatase (B) and Rutile (C) phase dominance after 24 hours .................. 48

Figure 5-8 SEM images of MC3T3-E1 Osteoblast on native and with nanostructure density having phase dominance from 5-7(B-C) after 48 hours .................................. 49

Figure 5-9 Fluorescence microscopy data of MC 3T3-E1 Osteoblast cell interaction on varying nanostructure densities with 24-48 hour incubation time (B \& E) Low density (C \& F) High

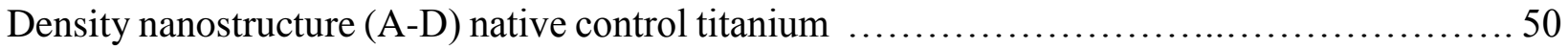

Figure 5-10 Change in MC3T3-E1 cell morphology due to both anatase and rutile dominant nanostructure after 24 hours $(\mathrm{A}, \mathrm{B}$ and $\mathrm{D})$ anatase enhanced $(\mathrm{C}, \mathrm{E} \& \mathrm{~F})$ rutile enhanced nanostructure. 51

Figure 5-11 Microvilli development in cells and effect of both anatase and rutile phase dominant structure in microvilli generation (A) - Normal microvilli development (B) - anatase dominant (C) - rutile phase dominant structures ................................................ 52

Figure 5-12 Reduction in cell adhesion with synthesized dominant phase .................. 53

Figure 5-13 Distinct change in cell morphology observed in osteoblast cells on synthesized structure from 24 to 48 hours 54

Figure 5-14 Influence of Synthesized nanostructure on NIH 3T3 Fibroblast cell trapping at rutile (A) and anatase (B) phase dominant nanostructure 55

Figure 5-15 SEM Images of NIH 3T3 Fibroblast cell interaction on native and with nanostructure density having phase dominance (B-C) after 24 hours 56 Figure 5-16: SEM Images of NIH 3T3 Fibroblast cell interaction on native and with nanostructure density having phase dominance (B-C) after 48 hours 57 
Figure 5-17 Development of filopodia on both anatase and rutile phase dominant nanostructure at 24 and 48 hours 58

Figure 6.1 Schematic illustration of laser material interaction A) laser surface patterning and B) laser nano printing 61

Figure 6.2 SEM/EDX line scan data of laser phase transformed surface pattern from $25 \mathrm{MHz}$ (high laser pulse frequency) to $4 \mathrm{MHz}$ (Low laser pulse frequency) holding a steady 1428 fs laser pulse irradiation width at a constant pulse per unit of $5 \mathrm{~mm} / \mathrm{s}$

Figure 6-3: EDX line graph showing the variation of titanium to oxygen with laser pulse repetition rate

Figure 6.4 SEM/EDX area scan data of laser phase transformed on nano-printed pattern from 25 $\mathrm{MHz}$ (high laser pulse frequency) to $4 \mathrm{MHz}$ (Low laser pulse frequency) at a steady $214 \mathrm{fs}$ laser pulse irradiation width having a constant pulse per unit of $5 \mathrm{~mm} / \mathrm{s}$

Figure 6-5: Area EDX graph showing the variation of titanium to oxygen with laser pulse repetition rate

Figure 6-6: Effect of Osteoblast cell differentiation of each synthesized surface pattern after 24 Hour incubation

Figure 6-7: Fluorescent microscopic image on effect of MC3T3-E1 osteoblast cell proliferation on each synthesized pattern (A-D) after 24 Hour incubation. (E)- Illustration of pattern synthesized with preferential cell attachment only on laser irradiated surface. 66

Figure 6-8: Fluorescent microscopic image on effect of MC3T3-E1 osteoblast cell proliferation on each nano printed pattern (A-D) after 24 Hour incubation. (E)- Illustration of nano printed pattern with preferential cell attachment only on laser irradiated surface.

Figure 6-9: Fluorescent microscope data on MC3T3-E1 osteoblast cell channeling, selective cell migration and repellence by nano-printed structure in 24 Hours. ... 68

Figure 6-10: Fluorescent microscopic image on effect of NIH3T3 fibroblast cell proliferation on each synthesized pattern (A-D) after 24 Hour incubation. (E)- Illustration of pattern synthesized with preferential cell attachment only on laser irradiated surface 
Figure 6-11: Fluorescent microscopic image on effect of NIH3T3 fibroblast cell proliferation on each nano printed pattern (A-D) after 24 Hour incubation. (E)- Illustration of nano printed pattern by USP laser having preferential cell attachment only on laser printed surface 


\section{LIST OF ABBREVIATIONS}

Å Angstrom,

AFM Atomic force microscopy,

BSA Bovine serum albumin,

BSE Backscattered electrons,

CVD Chemical vapor deposition,

DMEM Dulbecco's Modified Eagle Medium,

DMSO dimethyl sulfoxide,

EDS Energy dispersive X-ray spectroscopy,

fs Femtosecond,

HAZ Heat affected zone,

HEPS 4-(2-hydroxyethyl)-1-piperazineethanesulfonic acid,

LCVD Laser chemical vapor deposition,

MC3T3-E1 Osteoblast-like MC3T3-E1 cells,

MEMS microelectromechanical systems,

MEMS Microelectromechanical systems,

MHz Megahertz pulse,

MTT 3-(4,5-Dimethylthiazol-2-Y1)-2,5-Diphenyltetrazolium Bromide,

NEMS nanooelectromechanical systems

NIH 3 T3 Mouse embryonic fibroblasts,

nm Nanometer,

ps Picosecond,

SEM Scanning electron microscope,

TEM Transmission electron microscopy,

XRD X-Ray Diffraction,

$\boldsymbol{\mu m}$ Micrometer, 


\section{Chapter 1- Introduction}

\subsection{Evolution in Biomaterials}

Implantation is considered as the only possible route to treat complicated bone fracture. Conventionally, autograft, allograft, and xenograft implants are used to repair bone fractures and other bone defects. However, they are far from being ideal implants, as each procedure has its own specific problems and limitations [1-3]. Autografts are considered as the gold standards for bone replacement for many years, but they pose some severe concerns like donor shortage and donor site morbidity with infection, pain, and hematoma, which further limits their suitability and applications [4-6]. Allografts and xenografts always have the risk of disease transmission and immune response, and may cause loosening or complete loss of bone. Hence, as an alternative to all these several materials like metal, ceramics, and polymers have been introduced as artificial bone implants. Out of these metallic implants like titanium have gained popularity and have been able to reach real practical application [7-9].

Titanium implants provide the necessary strength and toughness that are required in loadbearing parts of the body, and due to these advantageous titanium continues to play an important role as orthopedic biomaterials in the current era. Although titanium implants are strong enough to meet the requirements for desired mechanical strength for an ideal implant, these traditional implant materials exhibit several problems, such as a short average lifetime (10-15 years), failure of implants due to implant loosening, inflammation, infection, osteolysis, and the release of certain ions and corrosion products (debris) at the site of implantation. [10-14] Also, titanium implants may lead to mechanical mismatch problems (stress shielding) between the implant and the adjacent bone tissue, where the integrity of bone-implant interface may be compromised due to the resorption of bone tissue. This short average lifetime (10-15 years) of traditional implants leads to much painful and expensive re-surgery to replace such failed implants [15-16].

Current trends in biomaterial research and development has utilized nano-technology to revolutionize certain features of implants to overcome the above said limitations. Material surface properties like surface chemistry, surface topography, electrical charge and crystallinity are identified to influence initial cell interactions and differentiation. Over the last few years, fabrication methods have been developed to understand the various interactions between cells and nanoscale controlled topographies. Some in vitro studies show different cell reactions with 
different surface roughness [17]. It was evidenced that rough surfaces encourage fibrin protein entrapment, which are important for osteogenic cells and mechanical stability between host bone and implants. [18]

Studies on vertically aligned $\mathrm{TiO}_{2}$ nanotubes reports a favourable cell response to nanotube diameters ranging from $15 \mathrm{~nm}$ to $100 \mathrm{~nm}$ and tailoring their diameters demonstrated a dramatic increase in cell viability with reducing nanotube diameters [19]. Therefore, most studies were focused on engineering implant surface to improve and accelerate osseointegration which ultimately increased tissue regeneration. [6-7]

In-spite recent progress, mechanisms dictating various cell responses on nanoscale structures is still in its infancy state where some underlying actions of cell repellence and apoptosis is yet to be fully analysed.

\subsection{Modern Nanofabrication Techniques}

Hitherto, various nanofabrication techniques like electro spinning, anodization, CVD, oxygen plasma treatment etc, have been conceived to synthesize oxide phases and nanostructures with accurate feature controllability. The synthesized structures does possess unique high surface to volume ratio, superior physiochemical properties and novel surface chemistry compared with their bulk counterparts. They are used in a number of other potential applications such as biosensors, drug delivery, biomedical implants, cancer treatment and tissue engineering. 


\subsubsection{Electro spinning technique}

Electro spinning has gained significant attention due to its ability to generate fibers with diameters as large as a few micrometers and as small as tens of nanometers [20]. The process requires a high voltage (typically 1 to $30 \mathrm{kV}$ ) to create a liquid jet and to push a viscous liquid through a charged metal needle. The grounded collector plate is placed at a very short distance (typically 10 to $20 \mathrm{~cm}$ ) below the needle [21]. As a droplet forms at the needle it becomes charged and electrostatic forces act on the droplet. Initially, the surface charges cause electrostatic repulsion of the droplet and then, the external electric field exerts a columbic force which shape the droplet into a feature known as a Taylor cone [22,23]. A jet is formed when the charged liquid overcomes

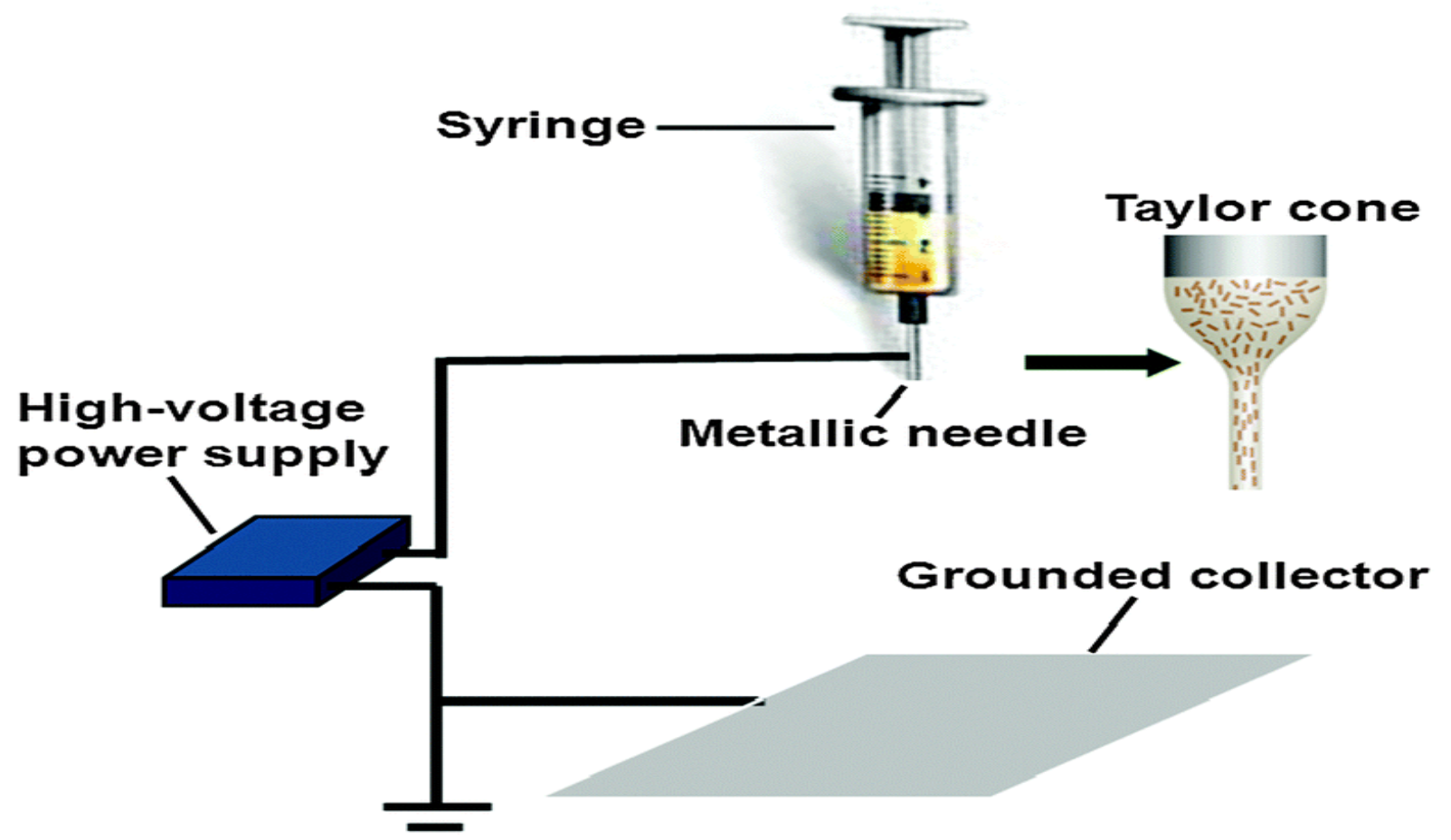

Figure 1.1 Schematic representation of electro spinning process [20]

its own surface tension and continues to stretch while it begins to evaporate into a fiber like morphology, causing the fiber to bend rapidly. The collector plate then attracts the fiber as it is deposited. A low viscous solution tends to break often during spinning resulting in spherical beads in the fiber. As can be seen in figure 1-2, patterns generated from titanium dioxide and by polymer [24]. 

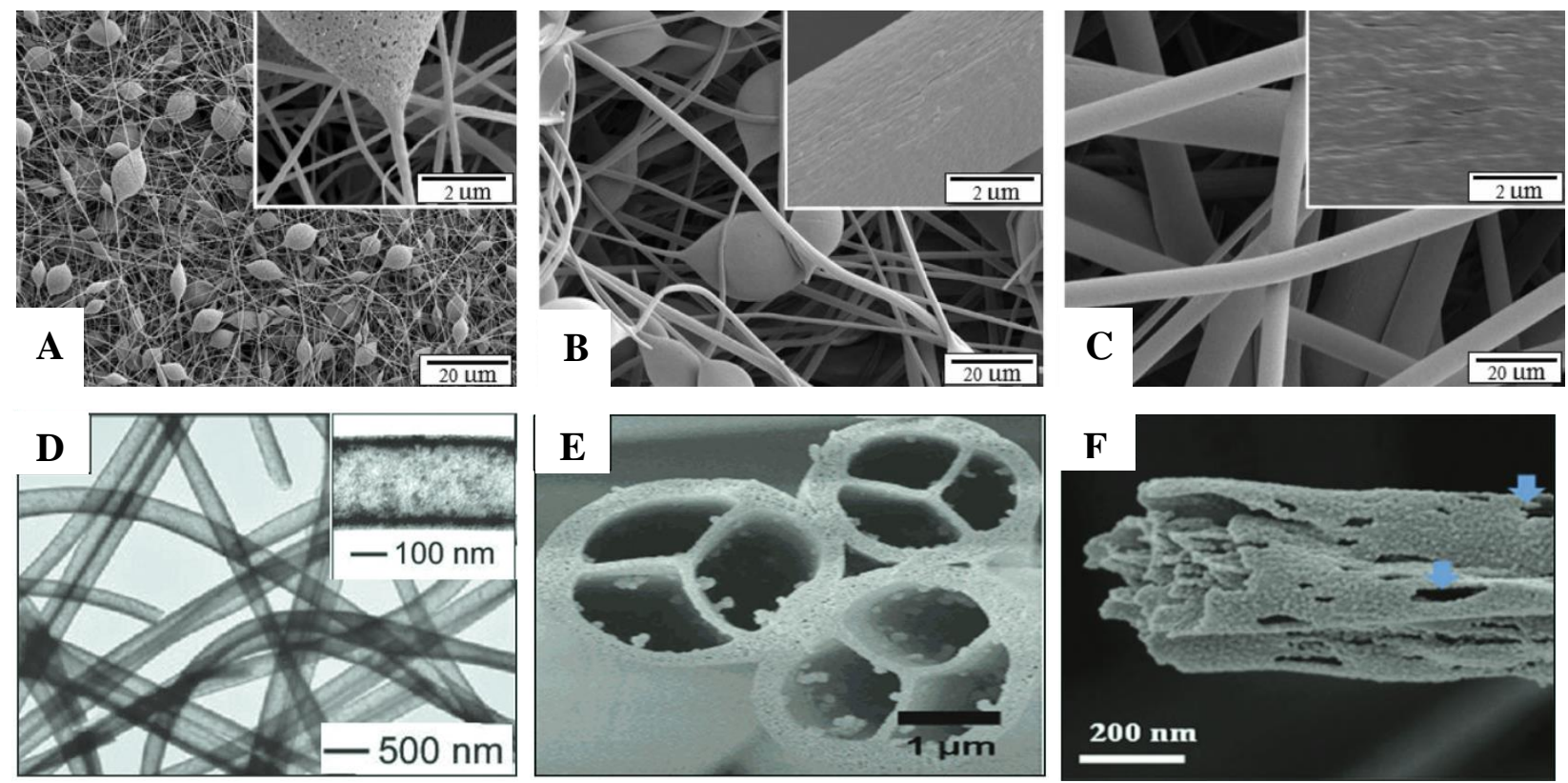

Figure 1.2 FE-SEM of electrospun polymer mixed titanium dioxide powder at A) 10 wt $\%$ B) 20 wt \% C) 30 wt \% with inserts showing higher magnification detail of individual fibers, D) E) and F) showing other surface patterns created using electro spinning[23,24].

Although, electrospinning has been used to generate composite metallic nanofibers, it is limited mostly to polymer nanofiber. Another disadvantages of electro spinning is limited control over generating 3-D nanostructures and using organic solvent [25].

\subsubsection{Anodization technique}

Fabrication of the titania nanotubes using anodization includes two steps. First it is basically a pre-treatment process, where Ti foil is anodized at some pre-set voltages for a period of time. This leads to formation of a surface oxide layer of nanotubes over the foil. Later, the nanotube layer is removed ultrasonically in deionized water, and the glossy underlying $\mathrm{Ti}$ is exposed. [26] 


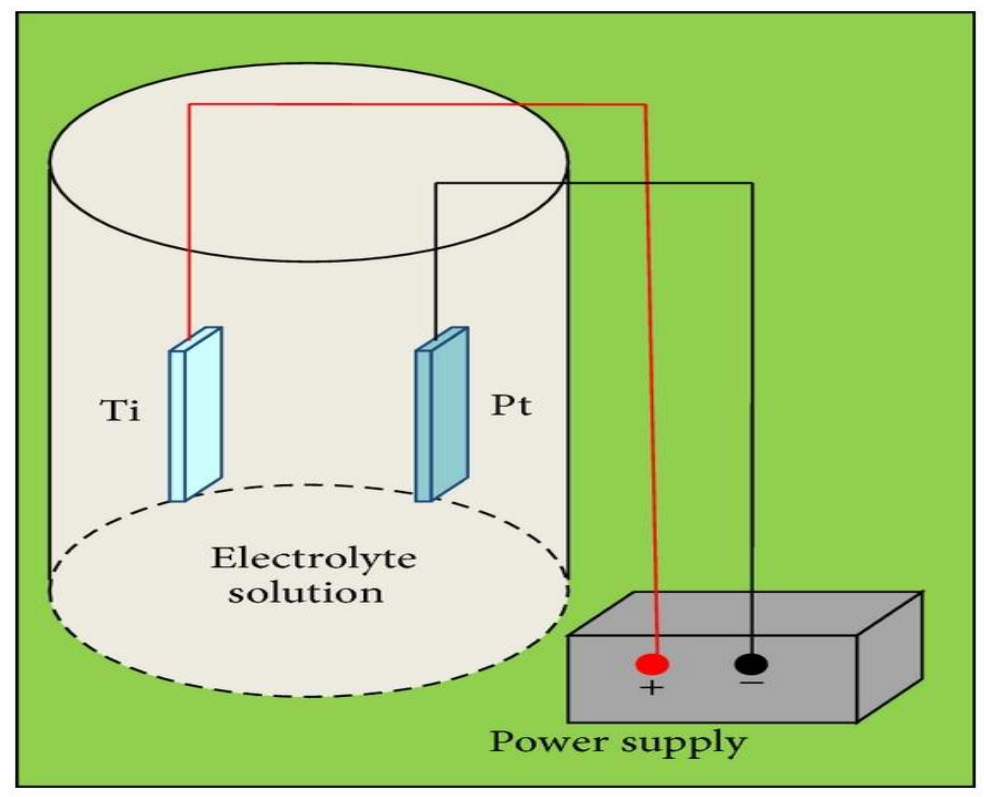

Figure 1.3 Schematic representation of titanium anodization [27]

The pattern left on the foil surface after the removal of nanotube layer, acts as a seed in further growth of well-aligned nanotubes. In the second step of the anodization, the pretreated titanium foil is used as the anode and Pt as cathode [27]. Then, after the conclusion of the two-step anodization process, the sample is cleaned ultrasonically in ethanol and finally rinsed in deionized water and ethanol. Although all traditional method uses hydrofluoric acid (HF) as an electrolyte, some aqueous electrolytes were also experimented. The nanotubes grows with all concentrations of electrolytes with increasing applied voltage. Hence in anodization, a multistep process is needed and material preparation is comparatively complex, owing to long duration and high temperature [28]. Also, as-anodized nanotubes are amorphous and a high temperature annealing step is required to form the crystalline phase [29-32]. 


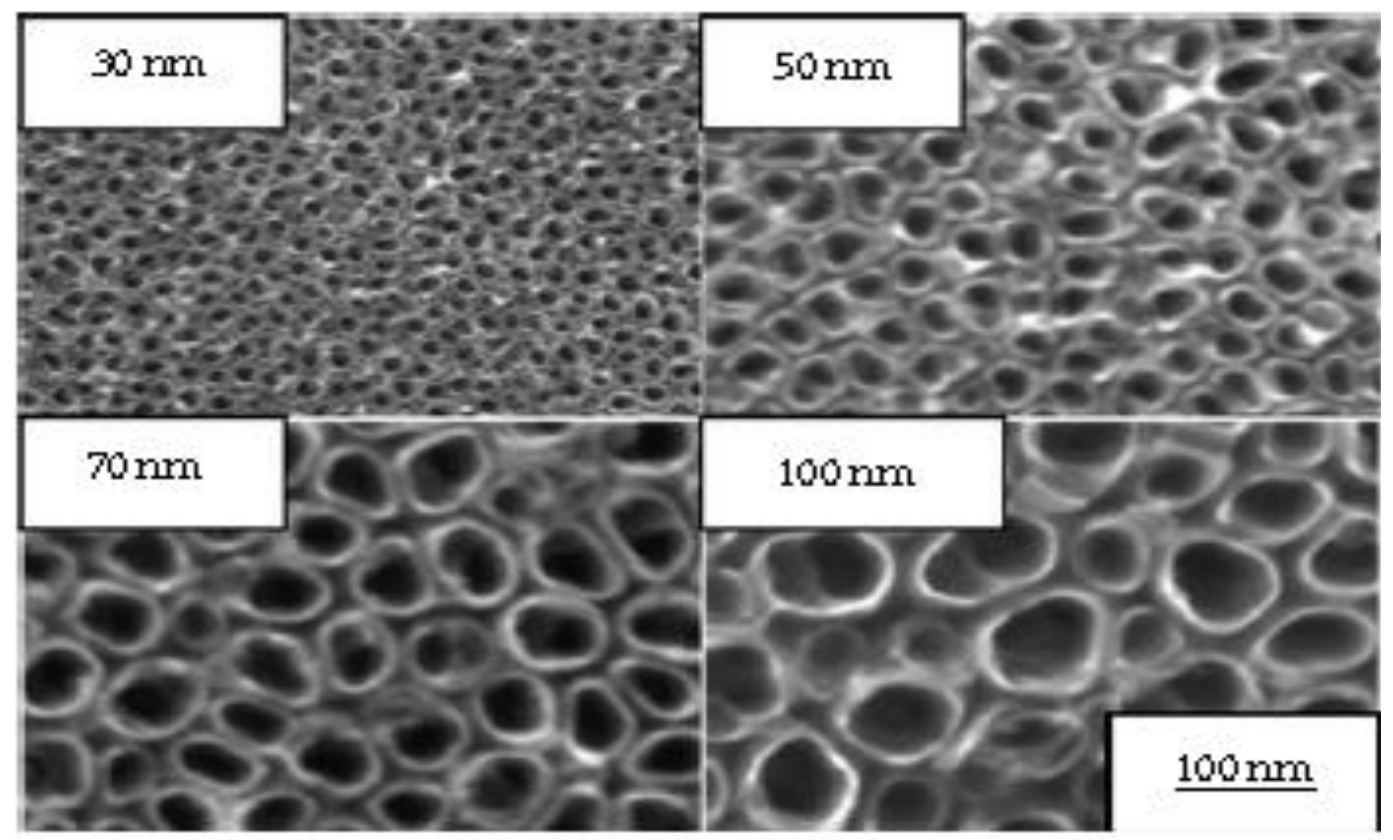

Figure 1.4 SEM micrographs of self-aligned $\mathrm{TiO}_{2}$ nanotubes with different diameter. The micrographs show ordered nanotubes with four different pore sizes between 30 to $100 \mathrm{~nm}$. [30]

\subsection{Functionalization of implant surface and cell interaction}

Alternative to surface topographic modifications and physiochemical treatments of surfaces to achieve enhanced cell implant integration, altering surface composition offers an unconventional path. Functionalization approaches of titanium oxides and its phases require a deeper understanding of biology and biochemistry of host tissue at the interface in terms of the mechanism by which cells adhere to surface [33] [34]. Titanium nanostructures have recently drawn great attention due to the exceptional physical, optical, and chemical properties they present, compared to that of their bulk counterparts which can be utilized for the development of different technological applications.

Precisely, titanium dioxide nanostructures have attracted great interest due to their outstanding mechanical properties, biocompatibility, and corrosion resistance [33]. They have been widely exploited in numerous applications including implantable drug delivery systems, biosensors, solar cells, tissue engineering and other medical devices. Titania nanotube films have been widely recognized as growth support substrates for bone, tissue and stem cells, for the prevention of bacterial adhesion, and enhancing blood clotting for control of haemorrhage. Recent 
in-vivo and in-vitro studies have demonstrated that surfaces comprised of nanotubes exhibit additional biological effects by producing integration between oxide and nanocrystals which also improves the cell-material interaction [37]. Titanium monoxide and its phases have shown great progress in implants as it was able to smoothly initiate apatite formation which is a fundamental pre-requisite for a biomaterial [38, 39].

\subsection{Concerns over other modern fabrication methods}

Various preparation techniques have been developed for the synthesis of titania nanostructures, such as anodization, chemical vapour synthesis, and template-based synthesis [30]. However, these traditional routes have disadvantages such as expensive precursors, long processing time and usage of toxic chemical and uncontrollable phase transformation during chemical route. These drawbacks seem to be harmful to titania nanostructure practical applications. But the processes involved in fabricating these nanoscale controlled structures were complex and often involved use of harmful chemicals as precursors with no prospective for tailored mass fabrication.

\subsection{Previous studies on laser synthesis of titanium}

In our laser micro and nanofabrication lab, it was first forecasted by Tan and Venkatakrishnan in 2008 that it may be possible to form nanofibrous structures from titanium substrate by femtosecond laser ablation with USP laser at ambient room conditions. This theory was based on some similar experimental results from irradiating materials like graphite, lead, aluminum, tin, and glass. The study was then continued by Sivakumar who found that irradiation of Titanium can lead to a formation of rutile phase structures and generalized a threshold laser fluence for titanium nanostructure formation. Later, Tavangar in 2011 applied $\mathrm{TiO}_{2}$ nanofibrous structures for biomaterials application using 4, 8, $12 \mathrm{MHz}$. These parameters were thought to have varied surface porosity which made cell adhesion possible. Both rutile and anatase phases were found by X-ray diffraction analysis [39].

However, these synthesis studies were severely lacking several optimization factors both in terms of generation and point of application. Later molecular sensor application was derived from these preliminary experiments by Dmitry in 2012 where the nanostructure network study was 
crucial in understanding the effects of confocal detection volume for Raman scattering [40]. The irradiation times could had a significant impact on detection capacity by the nanofibrous network. The knowledge built up from all previous work on $\mathrm{TiO}_{2} 3-\mathrm{D}$ network nanofiber synthesis to develop a functioning molecular sensor for Raman spectroscopy and implant application laid the fundamental platform for this thesis. The true potential application of both the functionalized phases and synthesized nanostructure is harnessed for various biomedical implants application for bone, coronary heart stents, pacemakers and even lab on a chip.

\subsection{Research Objective}

In addition, experimental studies are needed to examine the functionality of the synthesized nanostructures for different applications, in particular biomedical application. The presented research in this dissertation thesis aims to cover above mentioned required studies.

The main objectives of the dissertation can be highlighted as follows:

1. Analysis of ultra-short femtosecond laser ablated titanium and the application of phase transformed titanium for biomedical application.

2. Characterize the functionalized titanium nanostructures and study the synthesized biofunctionalized titanium nanostructures for smart implants

3. Utilizing synthesized phase transformed surface and nano-printed structure towards cell manipulation 


\section{Chapter 2- Laser material interactions}

\subsection{Laser Beam Characteristics}

In general, laser beam can be assumed to have an ideal Gaussian intensity profile, which corresponds to the theoretical TEM ${ }^{00}$ mode. Unfortunately, the output from real-life lasers is not truly Gaussian. To accommodate this variance, a quality factor - M2 factor, has been defined to describe the deviation of the laser beam from a theoretical Gaussian. A low M2 value indicates good beam quality and ability to be focused to a tight spot.

As a light source, a laser beam offers several unique features for material processing. Mono chromaticity is one of the unique characteristic of laser light when comparing it with any other source. Since the absorption coefficient of different materials is highly wavelength dependent, by selecting the laser beam wavelength from deep ultra-violet to infrared, one can precisely control the material processing. Laser is coherent and all the light waves travel in the same direction at the same frequency and are always in phase. This allows to focus the laser beam very tightly down to micron level and provides precise laser processing. Another important characteristic of the laser beam that it has very small divergence. The beam divergence determines the spot size where high divergence leads to larger spot size and vice versa.

\subsubsection{Laser ablation}

Laser ablation is the removal of material from a target object by incident laser light. The characteristics of this ablation are dependent on the wavelength, $\lambda$, of the laser light the type of target material and the intensity, $I$, of the laser beam.

The wavelength of light also determines the energy, $E$, of that light via the well-known energy relation,

$$
E=\frac{h c}{\lambda}
$$

where $h$ is Planck's constant $\left(6.626 \times 10^{-34} \mathrm{~J} \cdot \mathrm{s}\right)$, and $c$ is the speed of light in a vacuum ( $2.998 \mathrm{x}$ $10^{8} \mathrm{~m} \cdot \mathrm{s}^{-1}$ ) easily illustrates that as wavelength increases the energy of a photon decreases. 
Different target materials will have different absorption coefficients, $\alpha$, that determine the amount of light absorbed by it. The inverse of the absorption coefficient, $\alpha^{-1}$, will determine how far into a material particular wavelengths of light can penetrate. For example, materials that have low $\alpha$ will appear transparent. The relation is given as [36],

$$
\alpha=\frac{4 \pi k}{\lambda}
$$

where $k$ is the extinction coefficient of a material ( $k>0$ light is absorbed, $k=0$ light travels lossless forever). Thus choosing a wavelength that has a minimum absorption depth will allow for more efficient ablation.

The intensity of a laser pulse can be calculated from the well-known equation,

$$
I=\frac{4 P}{f \tau_{\mathrm{p}} d_{0}^{2} \pi}
$$

where $P$ is the laser power, $\tau_{\mathrm{p}}$ is the duration of the laser pulse (pulse width), $f$ is the number of such pulses per second (also called pulse frequency), and $d_{0}$ is the laser spot diameter [36].

The removal occurs when enough heat is absorbed by the target that material begins to atomize or vaporize. Thus, to determine the smallest dimensions like a hole that can be tunnelled, the wavelength of laser light will play a large part in determining the resolution of machined features. For a circular beam the smallest laser spot diameter, $d_{\min }$ is [36],

$$
d_{\min }=\frac{4 l \lambda}{\pi D}
$$

where $l$ is the focal length of the lens, and $D$ is the diameter of the beam incident on the focusing lens.

In general, if the focused laser energy flux is in excess of $10^{11} \mathrm{~W} / \mathrm{cm}_{2}$, it will result in an optical breakdown in solids and the excitation of plasma-induced ablation. Thus ablation can be easily achieved for a $\mu \mathrm{J}$ level ultra-short pulse with a focal spot size less than $10 \mu \mathrm{m}$. The energy flux in the beam focus can be calculated by

$$
q^{*}=\frac{E \times(1-R)}{t_{p} \times\left(\pi \times r^{2}\right)}
$$


where $q^{*}$ is the energy flux for the laser pulse, $E$ is the pulse energy, $t_{p}$ is the pulse duration, $r$ is the radius of the focused laser beam spot and $R$ is the percentage of the total beam energy loss in the optical system [36,37].

\subsection{Ultrafast laser interaction with titanium}

A variety of interaction mechanisms may occur when applying laser to materials. There are specific material characteristics as well as laser parameters that can contribute to the diversity. For material characteristics such as surface roughness the ablation threshold, pulse duration, laser pulse repetition rate, wavelength, focal spot size, pulse energy and exposure time/scanning speed of the materials are the most important criteria [38]. Here, especially laser pulse repetition rate, pulse duration are very crucial parameter to determine the type of interaction and mechanism it follows. The spatial distribution of volumetric energy density generated by laser irradiation is controlled by the incident energy flux and the optical absorption and scattering properties of the materials. Both optical absorption and scattering play important roles in determining the spatial distribution of volumetric energy density deposited in the target $[39,40]$.

Metals like titanium possesses high absorption coefficient suggesting the optical penetration depth of the incident radiation is also high where the intense heat needs to transported through for an effective transformation.

\subsubsection{Mechanism of agglomerated nanoparticle network formation during fs laser irradiation of titanium}

The mechanism of USP (Ultra-short Pulse) laser ablation is totally different from the ablation mechanism of the longer pulsed lasers (order of ns or higher) and CW lasers. Typical interactions, such as thermal interactions and photo ablation, rely on linear photon absorption, while USP laser ablation is governed by laser induced plasma-mediated ablation, also known as laser induced ablation, when intensities of the pulses are high enough $\left(10^{11} \mathrm{~W} / \mathrm{cm}^{2}\right.$ for solids and fluids) [41]. For linear absorption by longer pulsed laser, the energy of a single photon is usually smaller than the band gap needed to ionize an electron. For USP laser, the extremely high intensity of powerful pulses leads to a saturated photon flux which causes multi-photon ionization and tunneling photo ionization in the materials. Multi-photon ionization can be realized when an 
electron absorbs two or more photons simultaneously to gain enough energy to cross the band gap at very high laser intensity and is thereby excited to the conduction band. Tunnel ionization is a process in which electrons in an atom (or a molecule) pass through the potential barrier and escape from the atom (or molecule) [42].

These effects result in the ionization of some atoms and molecules, thereby providing initial free electrons for the laser induced optical breakdown. Once one starting free electron has been generated, it can absorb photons and accelerates via "inverse bremsstrahlung absorption" (IBA) in the course of collisions with other charged particles. The absorption of the photon increases the kinetic energy of the free electron. After several such events, the kinetic energy of the electron exceeds the band gap energy and the accelerated electron strikes another atom and ionizes it by impact ionization, resulting in two free electrons each with less individual energy. These two free electrons, in turn, absorb more photons, accelerate, strike other atoms, and release two more electrons, and the recurring sequences of avalanche ionization lead to a rapid growth in the number of free electrons [43].

Thus for plasma plume to grow, the irradiance must be intense enough to cause rapid ionization, such that losses do not quench the electron avalanche. Recombination and free-electron diffusion from the focal volume are the main loss mechanisms during avalanche ionization. When the density of the free electron reaches to a critical value, optical breakdown happens. At the breakdown threshold the plasma formation is restricted to the focal region of the laser beam. Due to the expansion of the plasma, ablation fragments (ablated species) are ejected out of the interaction zone and ablation is achieved [44]. 


\section{Chapter 3- Experimental Procedures}

\subsection{Materials}

Phase transformed titanium and titanium nanostructures have attracted great interest due to their outstanding mechanical, biomedical, and corrosion resistance property. These have been widely exploited in numerous medical technologies including biomaterial implants, drug delivery, biosensors, solar cells, tissue engineering, and heart stents. They are widely recognized as growth support substrates for bone and tissue cells, which prevents bacterial adhesion, with enhancing blood clotting for control of haemorrhage. For the experimental studies on Titanium, commercially pure titanium having following dimensions of $27 \mathrm{~mm} \times 20 \mathrm{~mm} \times 3 \mathrm{~mm}$ flat bars were used in the experiment. The titanium flat bars were mechanically sanded using SiC grit papers ranging from (240-1200) to remove macro roughness and then further polished using $5 \mu \mathrm{m}$ grade aluminum oxide colloidal suspension medium to have mirror like surface. The polished samples were subjected to sonication with $50 \%$ acetone concentration and $100 \%$ ethanol for 15 minutes each to remove all embedded particles generated in the polishing process. It was then rinsed with Deionised water and air dried.

\subsection{Laser Experimentation}

For carrying out the required programmed synthesis, a diode pumped $\mathrm{Yb}$ doped fiber oscillator laser system was deployed. The system can produce pulses at a central wavelength of $1064 \mathrm{~nm}$ with varying pulse widths from $214 \mathrm{fs}$ to $1428 \mathrm{fs}$ at operating average power of $16 \mathrm{~W}$. This generated variable pulse width laser pulses is chosen from laser pulse repetition rates between 4 $\mathrm{MHz}$ to $25.07 \mathrm{MHz}$ that has different peak power and pulse energy depending on apt laser conditions. For a given pulse width the peak power generated for each laser pulse decreases with increasing laser pulse repetition rate from 4 to $25.07 \mathrm{MHz}$. Hence, the heat /energy transmitted also varies accordingly. 


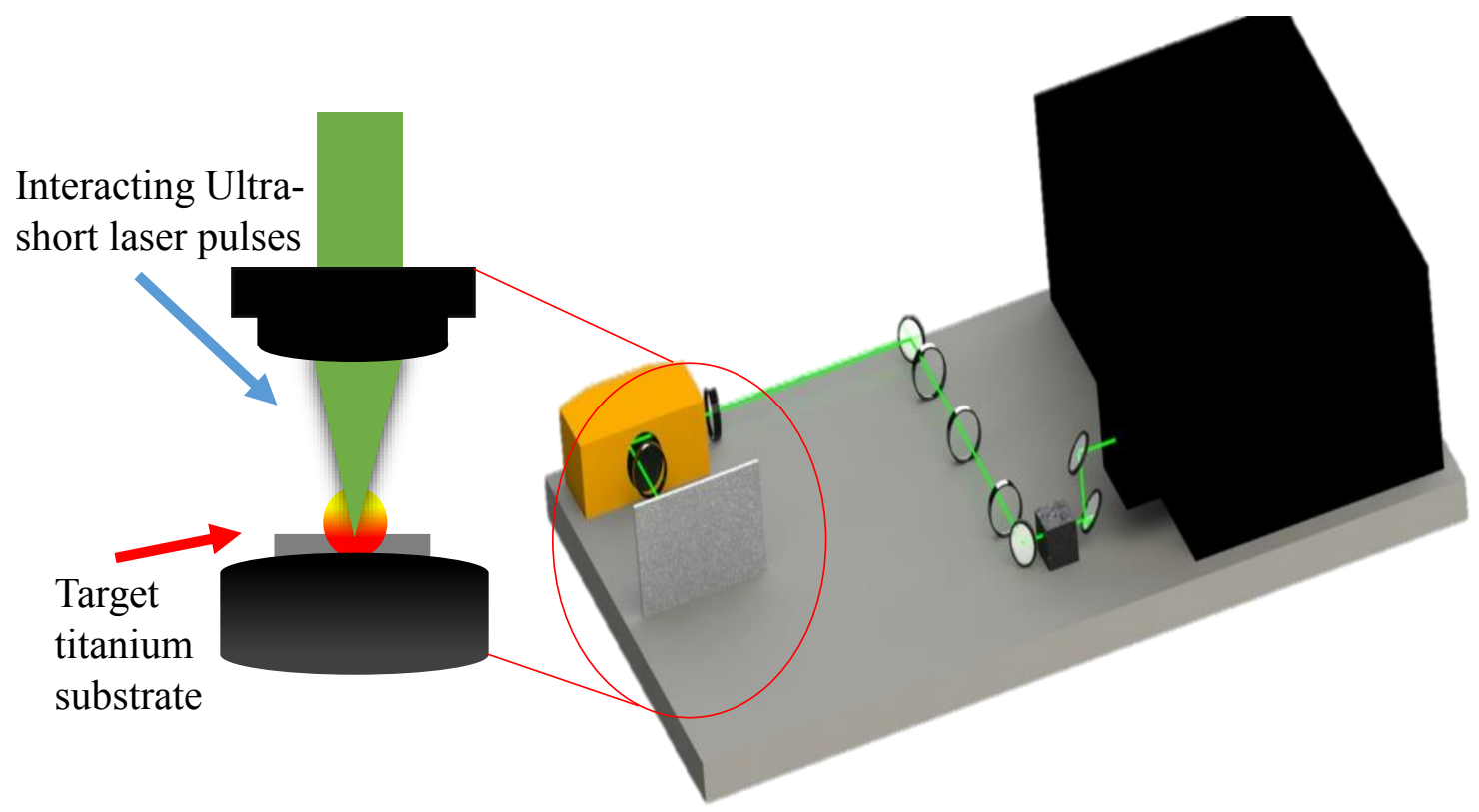

Figure 3.1 Schematic drawing of the experimental setup

A galvo scanner and telecentric lenses was used to focus the laser beam with $12.478 \mathrm{~mm}$ focal length. The theoretical laser minimum spot diameter $\left(\mathrm{d}_{0}\right)$ is calculated using equation 2.2. The laser beam focal length, laser pulse wavelength, and beam diameter are $12.478 \mathrm{~mm}, 1064 \mathrm{~nm}$ and $8 \mathrm{~mm}$, respectively. Thus, the theoretical spot size diameter was calculated to be $1.02 \mu \mathrm{m}$.

In the galvo scanner setup, the laser beam was expanded to $10 \mathrm{~mm}$ and then later decreased by a combination of a plano-convex $(f=-100)$ and plano-concave $(f=200)$ lenses; then the laser beam was rotated to circularly polarized by a quarter waveplate placed in the beam path. A telecentric lens with a focal length of $63.5 \mathrm{~mm}$ was employed to focus the normal beam on the surface of targets (see Figure 3.1). From equation 2.3 the theoretical spot size is calculated to be $10.38 \mu \mathrm{m}$ in diameter, when using $1064 \mathrm{~nm}$ pulse wavelength. During the experiment the spot size may be bigger due to scatter and misalignment.

\subsection{Sample characterization}

The microscopy and nanoscale morphology of the samples were studied with Scanning Electronic Microscope (SEM) and Transmission electron microscopy (TEM). Energy dispersive X-ray spectroscopy (EDX) analyses and X-ray Diffraction (XRD) analyses were used to investigate the chemical and phase characteristics of the laser irradiated titanium. 


\subsection{Cell culture experiments}

In an effort to develop a basic understanding of the initial stages of cell-surface integration, cell culture experiments were carried out with MC3T3-E1 and NIH 3T3 cell line mouse calvarian cells/osteoblast cells and Fibroblast cells from ATCC (American Type Culture Cultivation, USA). These have been shown to exhibit phenotypes that are similar to those expressed by human osteoblast and fibroblast. The cell culture experiments were performed on polished control surfaces, and laser phase transformed surfaces.

\subsubsection{Cell culture- MC3T3- E1 Osteoblast cell}

MC3T3-E1 mouse osteoblast cells cultured in a tissue culture flask with dulbeco modified eagles medium (DMEM), 10\% fetal bovine serum and 1\% penicillin/streptomycin maintained at $37^{\circ} \mathrm{C}$ in $5 \% \mathrm{CO}_{2}-95 \%$ air. The cells were split $1: 5$ whenever confluence was reached (which occurred after 5-7 days). The cells were harvested using cell scraper and the cells were then centrifuged at 980 revolutions per minute (RPM) down to the size of a pellet. Later they were resuspended in $4 \mathrm{~mL}$ of medium.

\subsubsection{Cell culture- NIH 3T3 Fibroblast cell}

Similarly NIH 3 T3 mouse fibroblast cells were cultured in a tissue culture flask with modified eagles medium (MEM), 10\% fetal bovine serum and 1\% penicillin/ streptomycin maintained at $37^{\circ} \mathrm{C}$ in $5 \% \mathrm{CO}_{2}-95 \%$ air. The cells were split $1: 5$ whenever confluence was reached (which occurred after 7-11 days). The cells were harvested using cell scrapper and the cells were then centrifuged at 980 revolutions per minute (RPM) down to the size of a pellet. They were later re-suspended in $4 \mathrm{ml}$ of medium.

\subsubsection{Preparing titanium surface for cell culture}

Prior to the seeding of the cells, the sample surfaces were cleaned and passivated. First the surfaces were sonicated for $15 \mathrm{~min}$ in acetone, followed by a $15 \mathrm{~min}$ duration in deionized water. After completion of the above process for each sample, each sample was again rinsed in distilled or deionized water for about 4-5 times and stored. At the time of commencement of the 
experiment, the samples were again rinsed 4-5 times in 75\% Ethanol solution and dried in a clean hood. This process was adopted so as to remove all the debris generated on the surface of the material and make it viable for conducting cell experiments.

\subsubsection{Fluorescent staining}

After $24 \mathrm{~h}$ and $48 \mathrm{~h}$ culture on the titanium substrate, cells were rinsed carefully with phosphate buffered saline (Ph-7.5) to remove the non-adherent cells. All samples were fixed with 4\% paraformaldehyde in PBS for 30 Min and then permeabilized with 1\% triton X-100 mix with glycine for $20 \mathrm{~min}$. For actin staining, the cells were incubated with $250 \mu \mathrm{L}$ of Alexa Fluor 488 (Life technologies, Canada) in 5ml of PBS and Dapi dye for staining nucleus. Cells were imaged on Nikon TE 2000 epifluorscence microscope using FITC and DAPI filters. 


\section{Chapter 4 - Novel Phase/ Non-stoichiometric oxide phase for cell controllability}

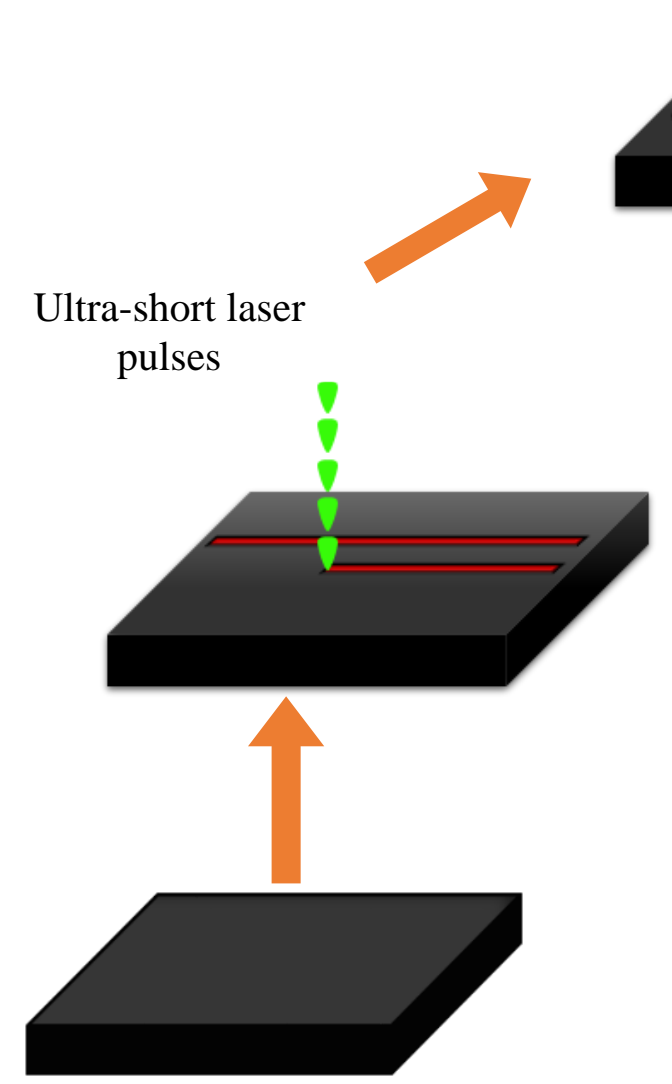

Titanium

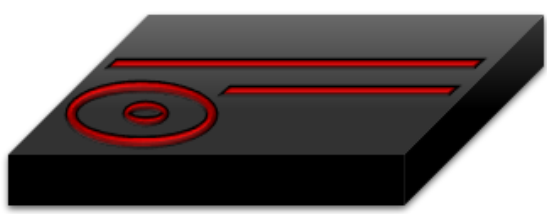

Phase Transformed surface
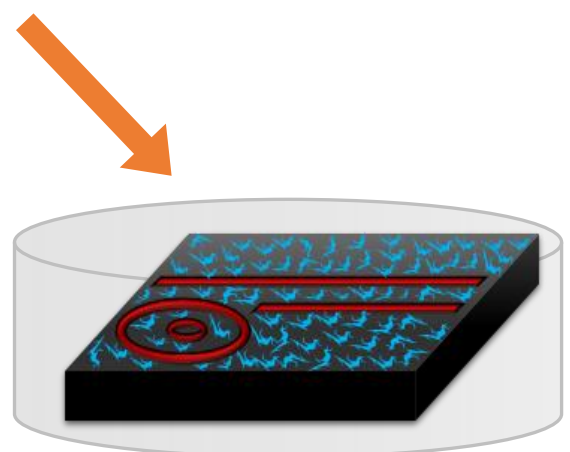

Cell Culture

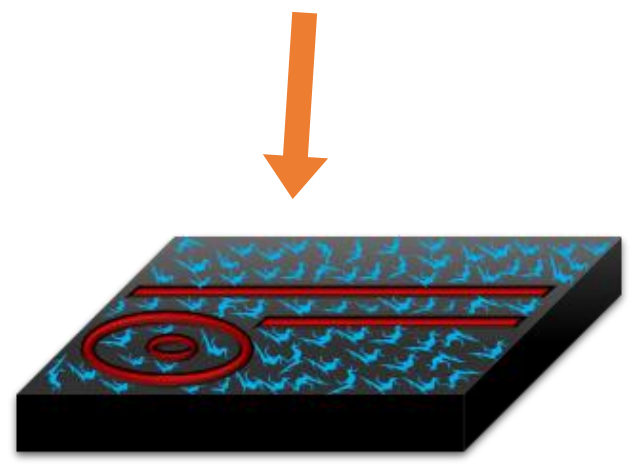

Cell activity on Phase Transformed surface

Schematic process diagram of non-stoichiometric oxide phase transformation by ultrashort femtosecond laser for cell controllability

The above process schematics explains ultra-short laser pulse interaction with titanium and nonstoichiometric oxide phase transformation. The phase transformed titanium surface is then induced to cell culture in an effort to test the synthesized non-stoichiometric oxide phases towards cell manipulation. 


\subsection{Introduction}

This chapter demonstrates the distinctive fabrication and functionalization of titanium substrate using USP laser by oxide phase transformation. It consist of a fundamental study which details oxide phase formation during USP laser irradiation in atmospheric conditions. The synthesized oxide surface was made to interact with primary osteoblast and fibroblast cells which then acts as a viable ECM platform for cells to functionalize thereby proving its versatility as a smart interactive biomaterial.

\subsection{Novel Laser material transformation}
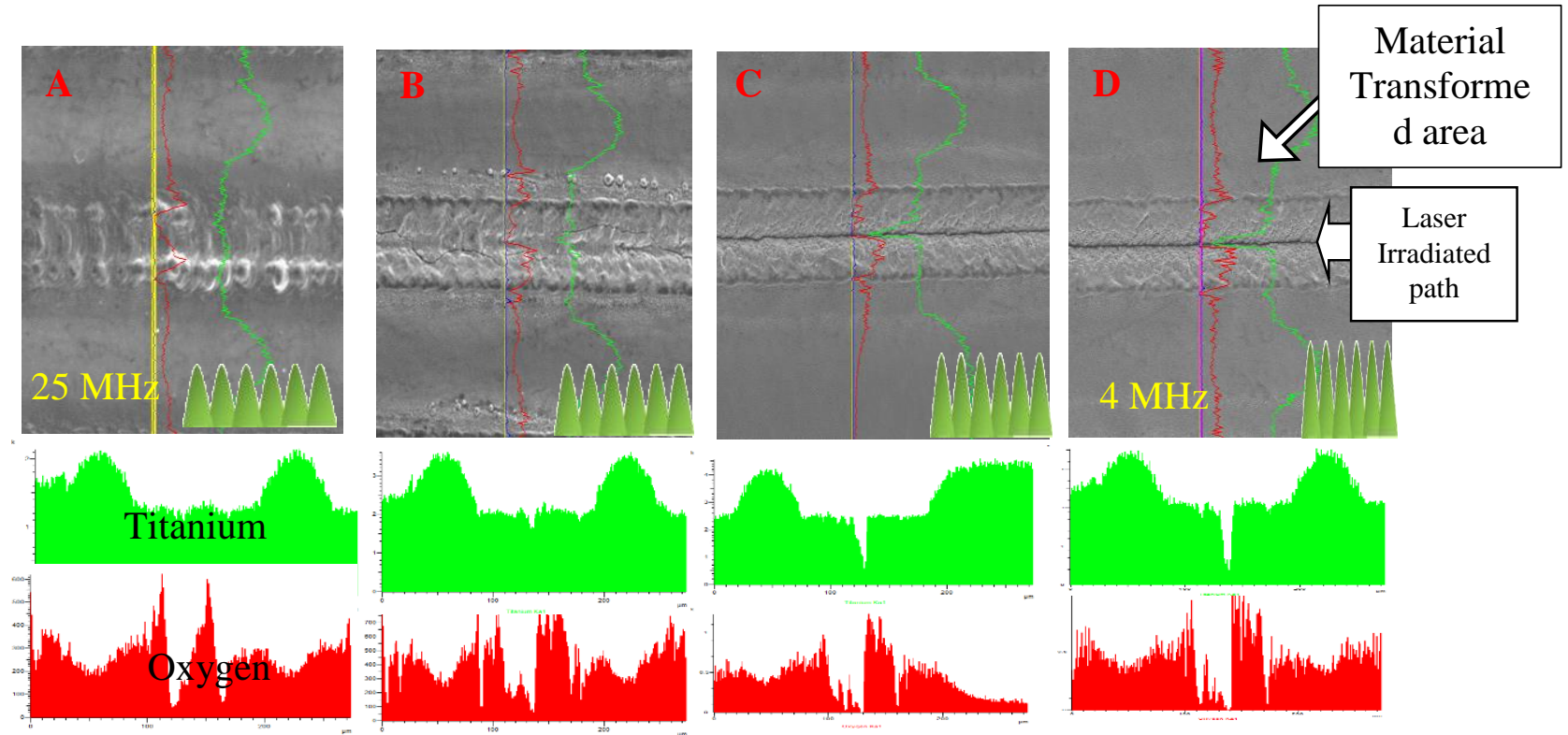

Figure 4-1 (A-D) Illustrates progression of SEM/EDX line scan of laser transformed titanium surface from $25 \mathrm{MHz}$ ( High laser pulse frequency) to $4 \mathrm{MHz}$ ( Low laser pulse frequency) at constant pulse to pulse separation time ( pulse width-214 Fs ).A constant pulse per unit area of $10 \mathrm{~mm} / \mathrm{s}$ ( scanning speed) is maintained. The green pulses in (A-D) represent the intensity or peak power generated per pulse at the above laser condition.

The above figure 4-1(A-D) shows gradual material transformation intensity by USP laser with respect to both topographic and intensity variation from the surface. The EDX line scan images also show the change in titanium to oxygen ratio across each laser irradiated path where sharp variations are observed around the material transformation area with changing topography. The material transformed surface at figure 4-1 (A) show more recast melt than that of figure 4-1(D) 
indicating the effect of transformation intensity by each laser pulse is more in figure 4-1(D) making the resultant topography appear smooth. The amount of oxidation is high in latter, where variation in oxygen to titanium seems high from the EDX intensity peaks. A dramatic dip in EDX intensity peaks is observed at figure 4-1(D) comparing with figure 4-1(A) suggesting acute change in features at the laser irradiated path at low laser pulse frequency (4 MHz).This is because, the energy realized per pulse at low laser pulse frequency $(4 \mathrm{MHz})$ is exponentially high compared to high laser pulse frequency (25 MHz) condition where, more time is spent by each pulse on material transformation at low laser pulse frequency $(4 \mathrm{MHz})$ compared with high laser pulse frequency (25 $\mathrm{MHz}$ ) having same pulse to pulse separation time ( pulse Width). Calculation of peak power for the above laser material transformation conditions are tabled in Appendix A1.

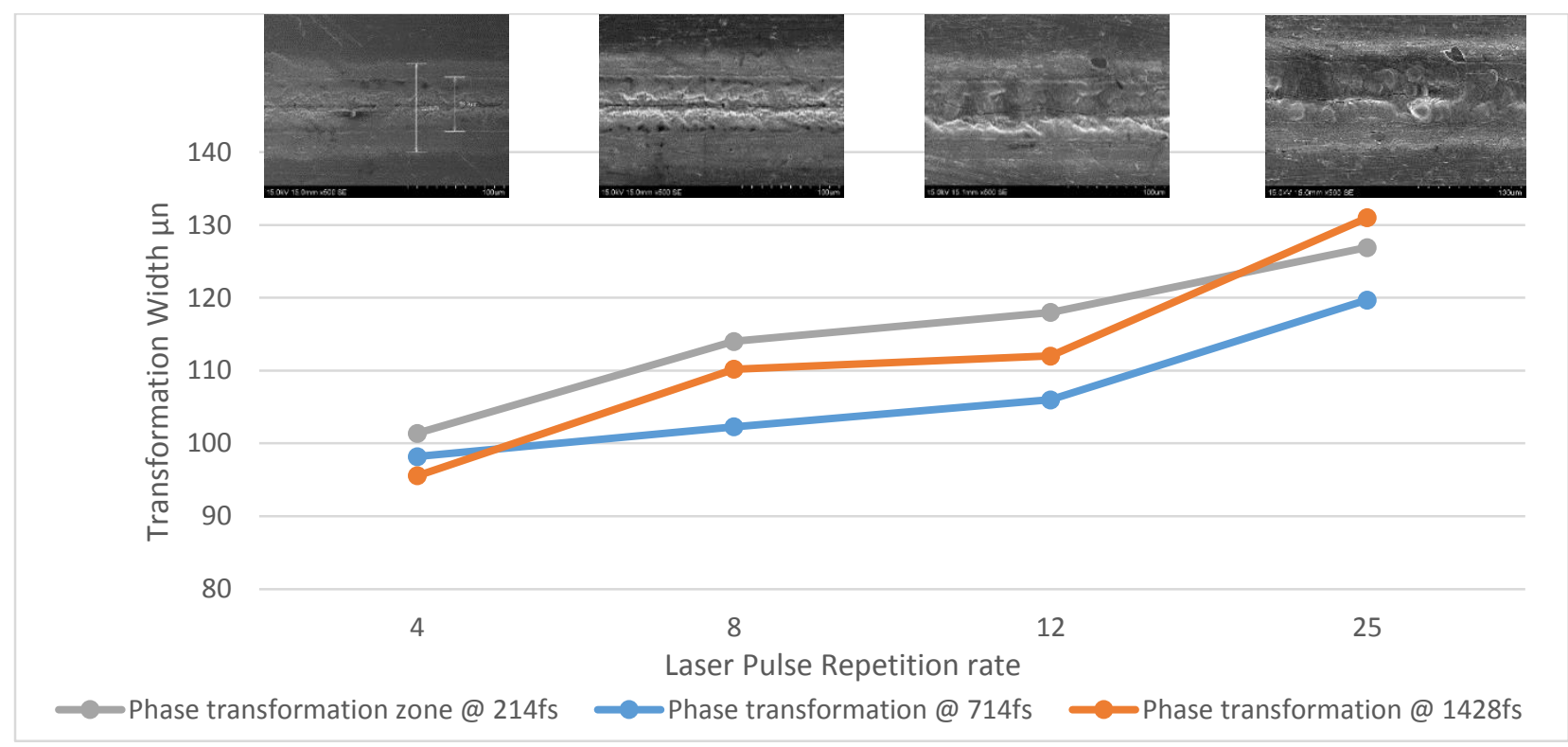

Figure 4-2 Shows extent of material transformation with varying laser pulse to pulse separation time at corresponding laser pulse frequency rates $(4,8,12 \& 25 \mathrm{MHz})$. A trend in transformation width with laser pulse repetition rate was also observed. The above SEM micrographs show transformation width @214fs

Although topographic variation can be observed on the laser material interacted surface, the width/ effective zone of transformation that formed around the interacted material needs to studied to accurately predict the transformation zone. From the above figure 4-2 one can now accurately predict transformation zone width as the laser pulse repetition rate is increased from 4 to 25.07 $\mathrm{MHz}$ the zone width also increases from 108.3 to $132.7 \mu \mathrm{m}$ indicating increased zone width. As 
pulse frequency increases it also increases the number of pulses exposes more surface to be transformed although the peak power of each pulse is comparatively low. The gradual increase in transformation zone for ascending pulse to pulse separation time also suggest the same where more power per pulse is effectively transmitted onto a target surface thereby linearly increasing transformation zone width.

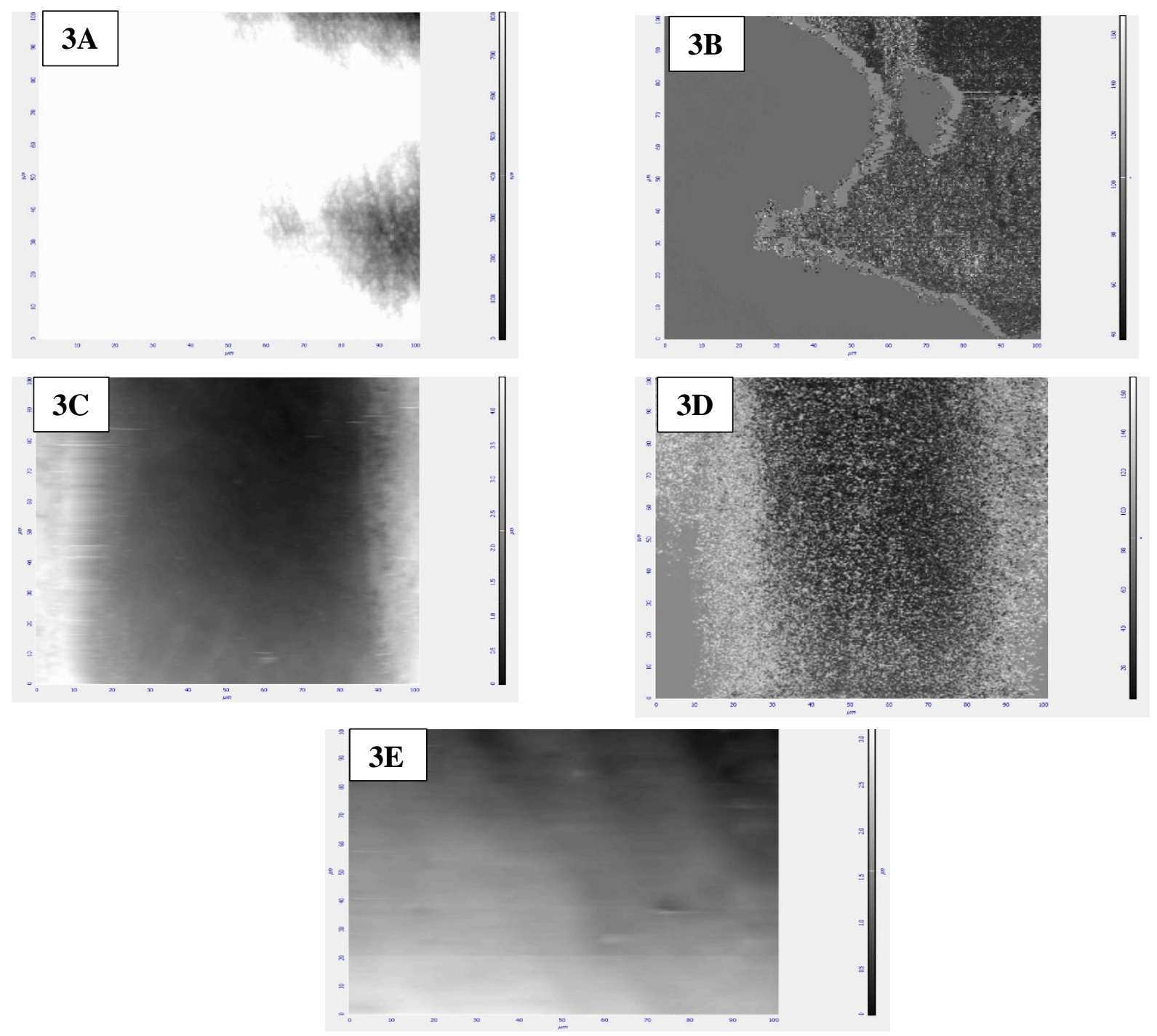

Figure 4-3(A-E) AFM scans of material transformed surface with native titanium substrate. Figure 4-3(E) is native titanium surface with topograhic or phase activity.Figure4-3(A-B) height and phase scan data at high laser pulse frequency rate(25 MHz) and figure 4-3(C-D) are height and phase scans of low laser pulse frequency rate( $4 \mathrm{MHz}$ ).

The above data shows change in material characteristics with sharp non-uniform peaks from the normal surface. The native titanium substrate had a relatively smooth morphology with no 
significant changes in the surface. But, both material property and morphology seems to change in material transformed zone from at high laser pulse frequency (25 MHz) which is in correspondence with EDX data from figure 4-1 (A). A pattern of acute non-uniform peaks is observed figure 4-3(B)-(D) during the scans which implies the presence of a complex mixture of oxides phases of varying intensities on the surface. The height image also shows an intense topographic variation on the target surface (having white contrast areas) in figure 4-3 (A)-(C). For USP laser, due to short exposure time, heat transmission into the substrate is expected to be longer than exposure time which results in inconsistent heat conduction. This ultimately results in inconsistent heat transmission from the laser irradiated area to adjacent heat transmitted zone. The depth saturation suggest prolonged laser irradiation time (i.e. scanning speed). Then, X-ray diffraction was used to analyse, quantify and investigate the effect of nano crystalline phases synthesized by USP laser.

\subsection{Phase Analysis in engineering novel polymorphic phase oxides}

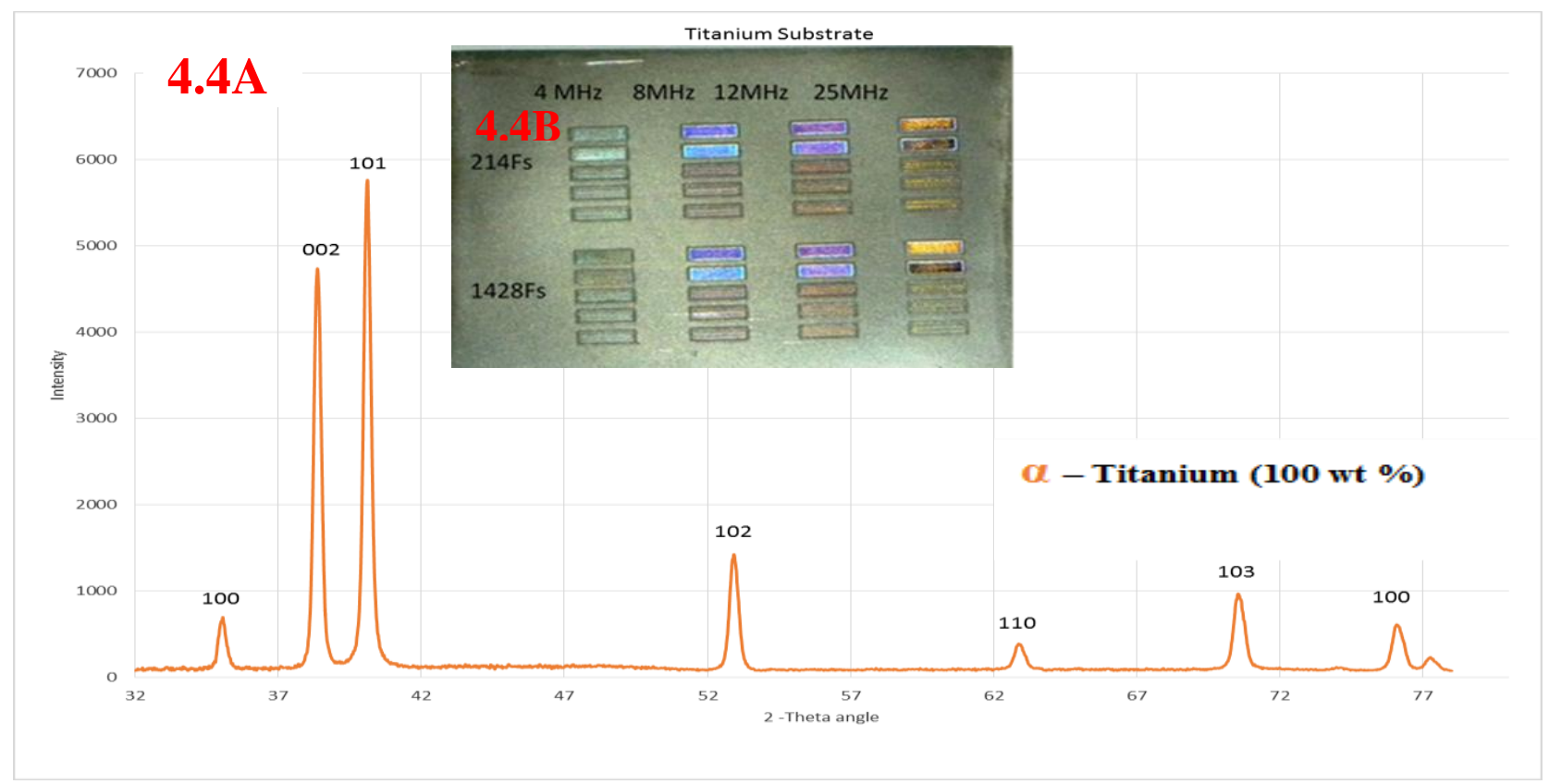

Figure 4-4A represents XRD spectrum of unmodified native $\alpha$-titanium substrate and 4B optical microscope image of the phase transformed surface. 

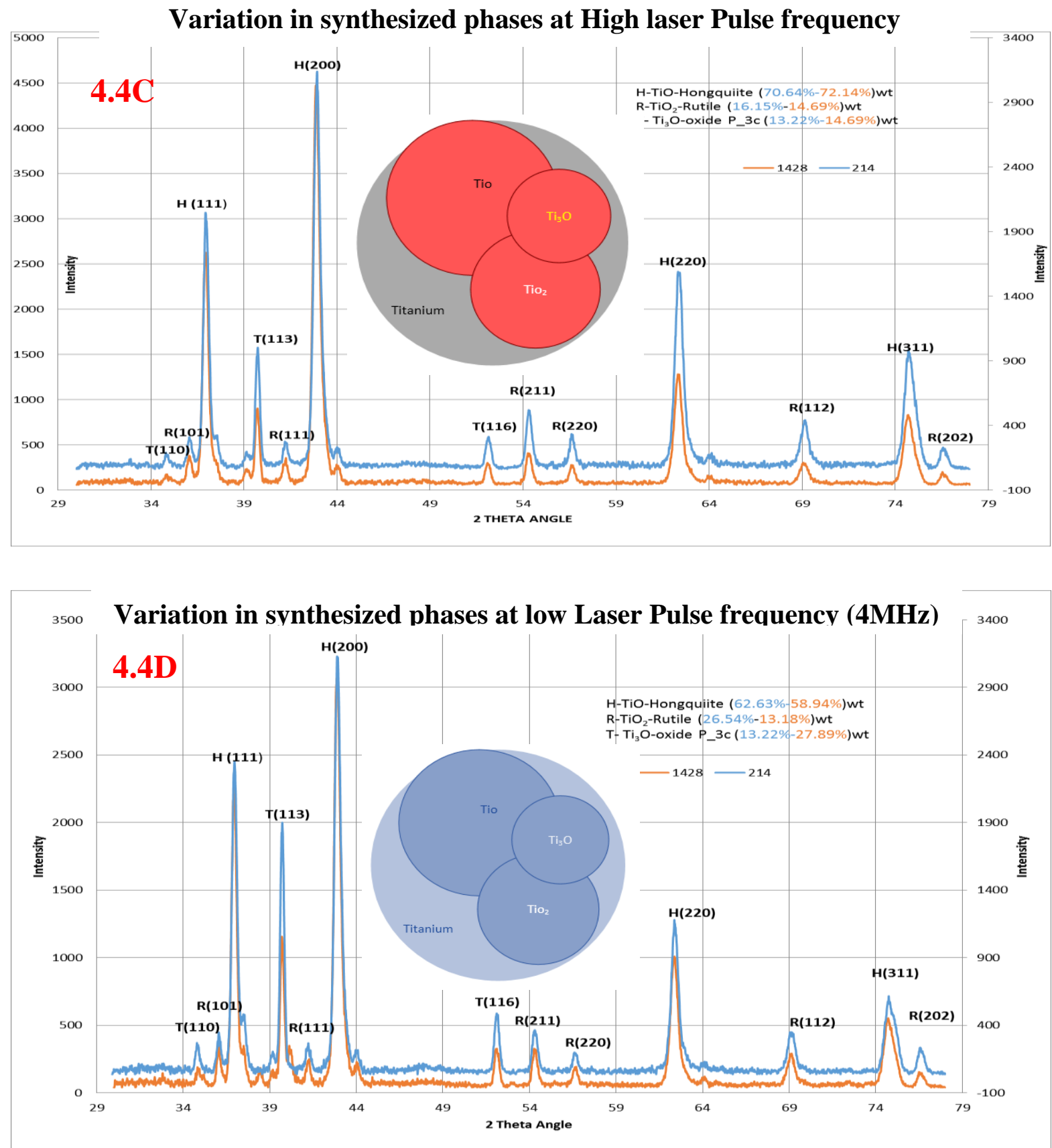

Figure 4-4C-D represents XRD spectrum of titanium oxides phases synthesized on titanium substrate at high (25 MHz) and low (4 MHz) laser pulse frequency.

Figure 4A shows the native control titanium sample entirely composed of ( $\alpha$-titanium) in Hexagonal close pack (HCP) structure. The native titanium had (100), (002), (101), (102), (110) and (103) diffraction peaks with $2 \theta$ angle around $35^{\circ}, 38^{0} .40^{\circ} .53^{0}, 63^{0}, 71^{0}, 76^{0}$ respectively.[4547] But, in figure $4 \mathrm{C}$ the intensity and $2 \theta$ angle of the phase transformed surface indicates a polymorphic phase transition of $\alpha$-Ti into explicit oxides with specific weight ratios. Compared with native titanium substrate, phase transformed surface also displayed hongquiite phase which 
is considered as a rare structure of $\mathrm{TiO}$ oxide having peaks around at $37^{0}, 43^{\circ}, 62^{0}$, and $75^{0}$. [4547]. Titanium dioxide having only rutile phase was detected having orientations (101), (111), (211), (220), (112) and (202) [36, 37]. Also, Tri-titanium oxide $\left(\mathrm{Ti}_{3} \mathrm{O}\right)$ an intermediate compound oxide was found having orientations (110) (113) (116). During phase transition HCP $\alpha$-Ti has transformed into cubic $\mathrm{TiO}$ and $\mathrm{Ti}_{3} \mathrm{O}$ oxide. Then, at sequentially later stage, rutile $\mathrm{TiO}_{2}$ oxide is formed acting as a passive layer over the laser irradiated surface and thereby stopping further oxidation. The final products after polymorphic phase transformation reaction is a mixture of aforementioned three oxides. At high laser pulse frequency $(25 \mathrm{MHz}$ ) having $214 \mathrm{fs}$ as pulse to pulse separation time figure 4-C TiO oxide is found to be dominant ( 70\%) and when this pulse to pulse separation time is increased to $1428 \mathrm{fs}$ the $\mathrm{TiO}$ oxide peak intensity further increased by 2 percent with simultaneous $\sim 2 \%$ reduction in rutile phase. Since, the programmed laser pulses efficiently synthesized more Hongquiite phase we can call this Hongquiite functionalized phase. But, at low laser pulse frequency (4 MHz) from Figure 4-D with increased pulse to pulse separation time lead to dip in $\mathrm{TiO}$ oxide and increase in Rutile phase. This is due to peak power generated by each pulse getting transmitted into the material more effectively. As Rutile phase see a dramatic transformation with varying pulse to pulse separation time, a similar trend pattern can be inferred for Rutile phase and can be called as Rutile functionalized phase However, no evidence of residual metallic $\alpha$-Ti or $\beta$-Ti is observed suggesting the functionalized surface is $\sim 10 \mu \mathrm{m}$ thick from X-ray diffraction penetration limit. The X-ray diffraction data from Figure 4(A,C,D) clearly indicates an oxygen diffusion limited reaction process on the material. This conclusion can be drawn from following two assumptions. (i) Phase transition could be initiated only in molten melts where oxygen has high solubility through solid metals. (ii) Primary products from transition metal are monoxides, rather than a thermodynamically favored dioxides at high pressure and temperature. The polymorphic phase oxides realised here cannot exist under normal equilibrium conditions. The co-existence of multiple oxide and its phase has created a series of interfacial boundaries and a variety of phase transformation reaction that has occurred simultaneously at different regions within the interacting surface. These secondary reactions are imperative to the entire dynamics of phase transformation process because, part of the monoxides synthesized react with excess oxygen in air to form stoichiometric stable $\mathrm{TiO}_{2}$ oxides. 
Then, consecutively, the inner interface between monoxide and substrate titanium reacts to form even lower suboxides like $\mathrm{Ti}_{3} \mathrm{O}$. This is consistent with the low temperature regime as found in the Ti-O phase diagram [48]. Since, only a small amount of rutile $\mathrm{TiO}_{2}$ were synthesized on the surface the mechanism of phase transformation is kinetically controlled by oxygen diffusion from the atmosphere by programmable laser pulses and thus becomes either Hongquiite or Rutile phase functionalized surface.

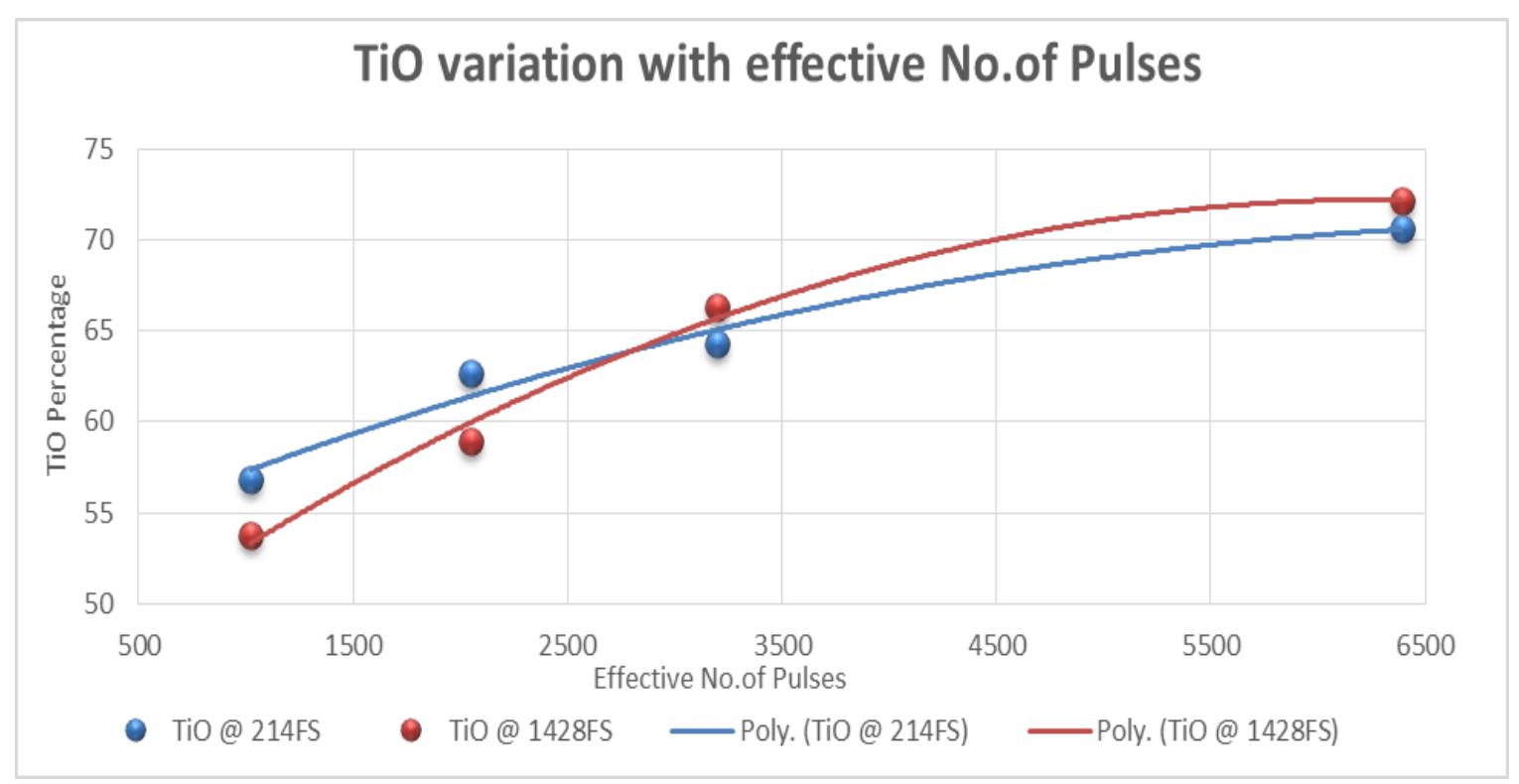

Figure 4-5 Graph representing magnitude of $\mathrm{TiO}$ oxide synthesis with cumulative pulses.

A noticeable color change can also be seen on the functionalized surface at different irradiating conditions. Earlier fundamental studies on titanium laser irradiation concerning color to composition ratio suggested, synthesis of $\mathrm{TiO}$ oxide to Golden color and deep blue and violet to $\mathrm{TiO}_{2}$ oxide [49]. Similarly, a range of color palette is found to be coexisting in the synthesized specimen which seems to be in congruent to the proposed suggestions. However, colored titanium oxide have not found much application other than jewellery and paint.

Since TiO oxide generated is found to be dominant phase Figure 4-5 shows the trend in cubic TiO oxide variation with respect to effective no of laser pulses. The oxide generation is dependent on the peak power initiated by each laser pulse. As the number of pulses increases having the same pulse to pulse separation time (i.e. pulse width), the surface temperature at the target interacting with laser increases, thereby producing more non-stoichiometric cubic TiO oxide with part of it later undergoing further oxidation to form passive stable $\mathrm{TiO}_{2}$ rutile phase. The above trend line graph also recommend that as the cumulative pulses reaches a maximum where $\mathrm{TiO}$ oxide 
generation saturates suggesting that almost all free oxygen found in the plasma during laser interaction is utilized for TiO oxide generation [48].

\subsection{Influence of synthesized phases on cells}

Several studies have related the growth of both MC3T3-E1 osteoblast and NIH 3T3

fibroblast cells to the Titanium oxide phases synthesized. Rutile phase is reported to enhance the growth of both osteoblast and fibroblast cells.

\subsubsection{Influence of osteoblast cells on functionalized phases}
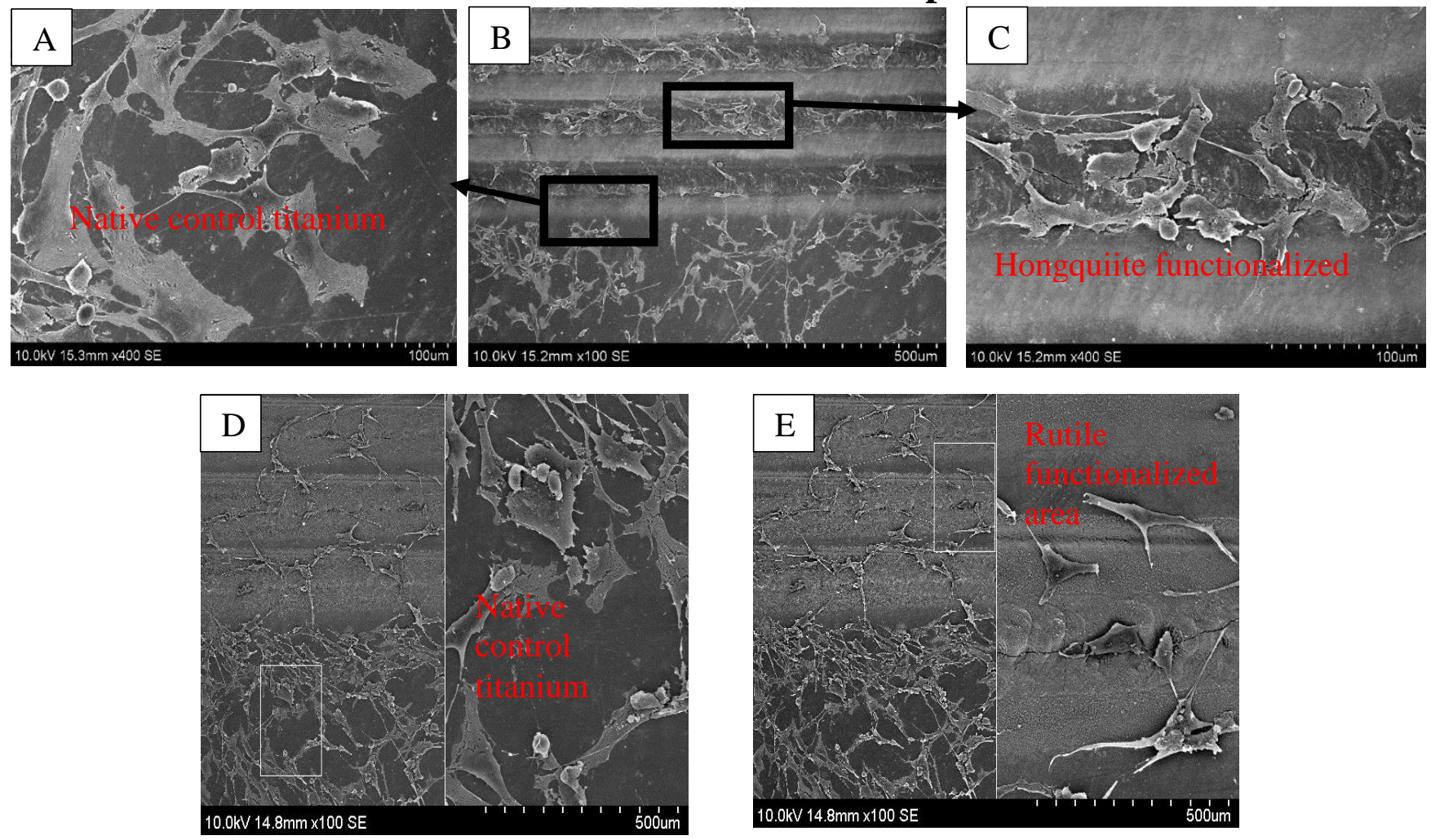

Figure 4-6: SEM images of MC 3 T3 osteoblasts on native titanium and phase transformed surface after 24 hours. On the native Titanium reference surface cells are flat and attach with the whole body whereas Figure $(C \& E)$ on phase transformed surface cells adhere exclusively on top of the laser irradiated surface.

The cracks on the laser interacted material on figure4-6(C\&E) were observed to have cells likely due to excessive stress on the material during laser material interaction and cell fixation. The SEM images reveal acute morphological differences of MC3T3-E1 osteoblasts on both phase functionalized surfaces figure4-6(C\& E) in comparison to the planar titanium reference on figure 4-6(A).On the native Ti-reference, the cells demonstrate a flattened phenotype after $24 \mathrm{~h}$ and attach 
to the surface with their whole cell body. The cell shape of MC3T3-E1 osteoblasts on the phase transformed surfaces was different look elongated and expressed long cellular extensions. The first phase in cell-material interaction involves cell adhesion and spreading. Analysis of the initial cell adhesion of MC3T3-E1 osteoblasts revealed a significant decrease of adherent cells on phase functionalized surface in comparison to the planar native Ti-ref, (24 hours) where spreading of MC3T3-E1osteoblast cells was significantly impaired during 24 hours $(p<0.001)$ when compared to the native Ti-ref figure 4-6(A\&D). On all phase functionalized surfaces the cell area was less than half as compared to the control cells. The lower cell spreading on functionalized substrates is not all caused by cell death/apoptosis.

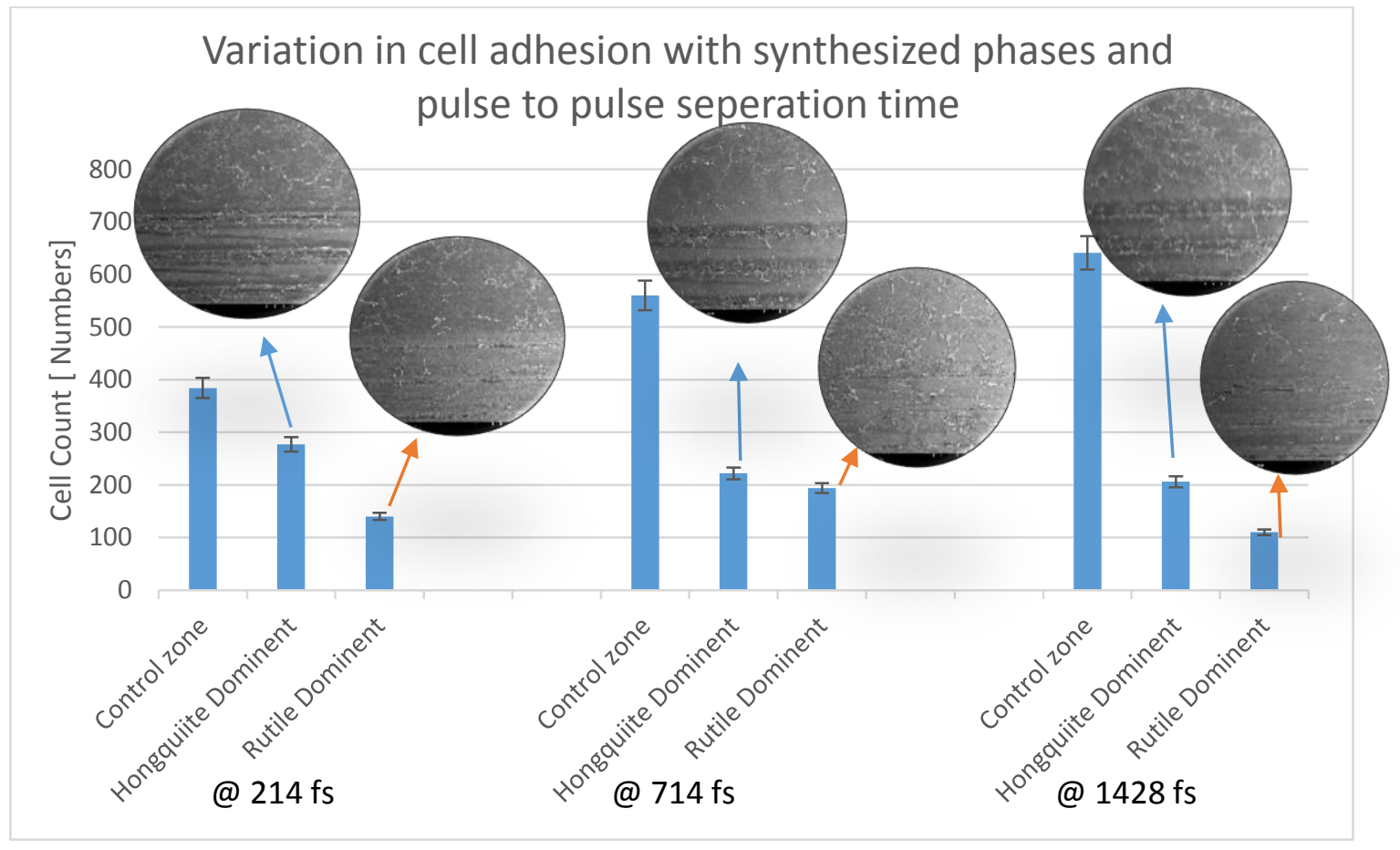

Figure 4-7 Adhesion of MC 3T3 Osteoblast cells on both phase dominant surfaces in relation to planar reference titanium. Note that cell adhesion after 24 hours incubation significantly reduces on all rutile dominant phase surface. As pulse to pulse separation time is increased from 214fs to $1428 f$, the effect becomes more pronounced with least cell adhesion on rutile dominant phase which could be due to the effect of cumulative laser pulses with peak power.

Thus when the performance of cell adhesion on phase functionalized surface is compared across the three pulse to pulse separation times in figure 4-7 (214, 714, $1427 \mathrm{fs})$, only 214 and $1428 \mathrm{fs}$ conditions seems to yield some interest with both the dominant phase showing significant cell 
reduction compared with the native titanium substrate. Hence, in 48 hour incubation even more reduction in cell proliferation on both phase functionalized surface is expected (result shown in figure 4-8).

\subsubsection{Directional cell mobilization with dominant phase surface}
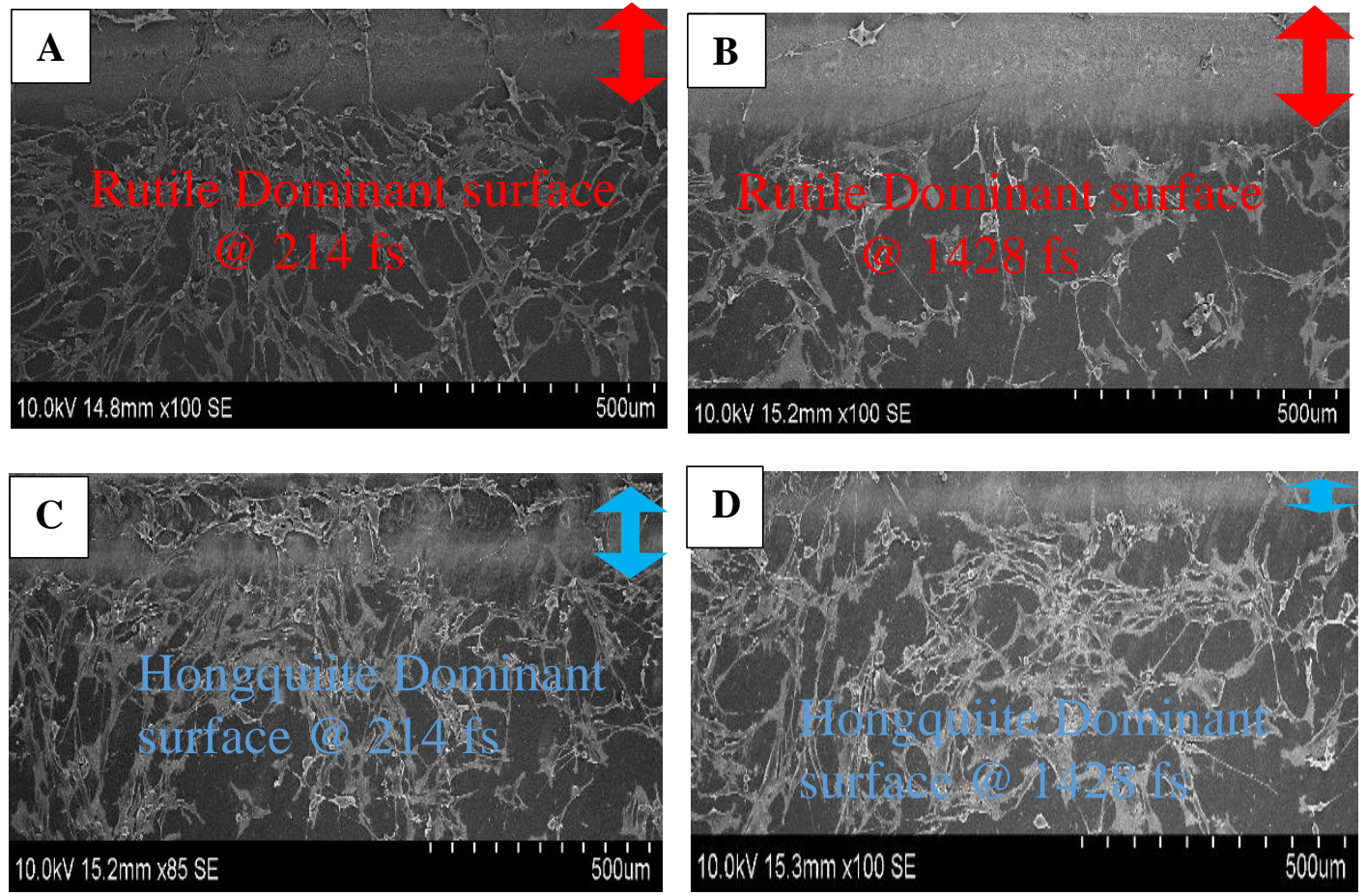

Figure 4-8 MC 3T3 Osteoblast cell activity for 48 hours incubation period on both hongquiite and rutile phase dominant surfaces at two pulse to pulse separation time(214- 1428 fs).

Based on the data from 24 hour incubation, MC3T3-E1 osteoblast cells cultured on both phase dominant surfaces for 48 hour demonstrated some clear morphological changes, as compared to the flat control surface as shown in figures 4-8(A)-(D). MC3T3-E1 cells adopted a more elongated shape and appeared more aligned near and on the phase dominant surface. This is consistent with all other studies showing that both grooved topography and oxide phases induces adherent cells to elongate and align [12]. The above SEM figures4-8(A-D) also shows that almost all MC3T3-E1 cell spread away from several phase dominant areas, indicating that the observed elongation and alignment were not a result of physical entrenchment of the cells in the channels but a physiochemical reaction between the substrate and cell which needs to studied in detail. The cells on the flat control surface adopted a random walk movement, in which cells migrated about 
from their starting position with no clear sense of direction. The above data thus suggests with slight topographical cue provided in the fabrication combined with sufficient physiochemical cues from the environment is needed to govern the migration routes of MC3T3-E1 cells.
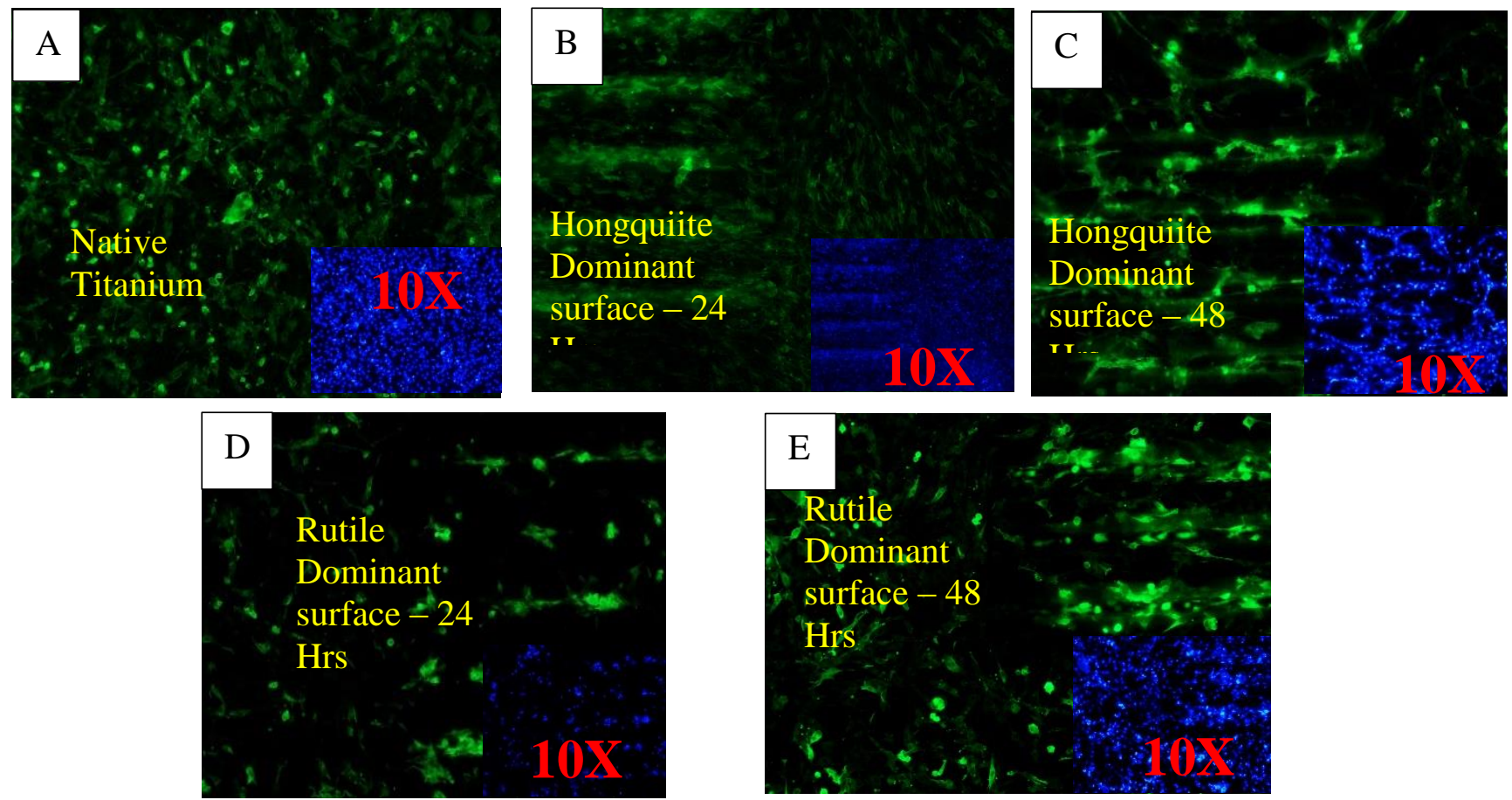

Figure 4-9(A-E) MC 3T3 Osteoblast cell activity for 24-48 hours incubation period on both hongquiite (B-C) and rutile dominant surface (D-E) compared to native titanium reference surface.

The first phase in cell adhesion/ attachment was quantified by cell spreading and proliferation. But, the dynamic process of cell- material dialogue and its subsequent survival on the interacting material can only be accurately quantified using cytoskeletal arrangement, focal adhesion complex, nucleus, proteins, integrin etc.,. The above results figure 4-9 confirm the effect of phase dominant surface on MC3T3-E1 osteoblast cells. The cytoskeletal arrangement near the functionalized surface was weak for the initial 24 hour incubation Figure 4-9 (B-D) for both the dominant surfaces and hence the MC3T3-E1 cells could never initiate proliferation on it further during 48 hours. This correlates with the nucleus image when no evidence on nucleus present close to the phase transformed material. As the incubation period was doubled cell proliferation dramatically increased on the plane titanium reference surface surrounding the phase dominant material but, no increase in cell proliferation was observed on both phase dominant materials from Figure 4-9 (C \&E). 


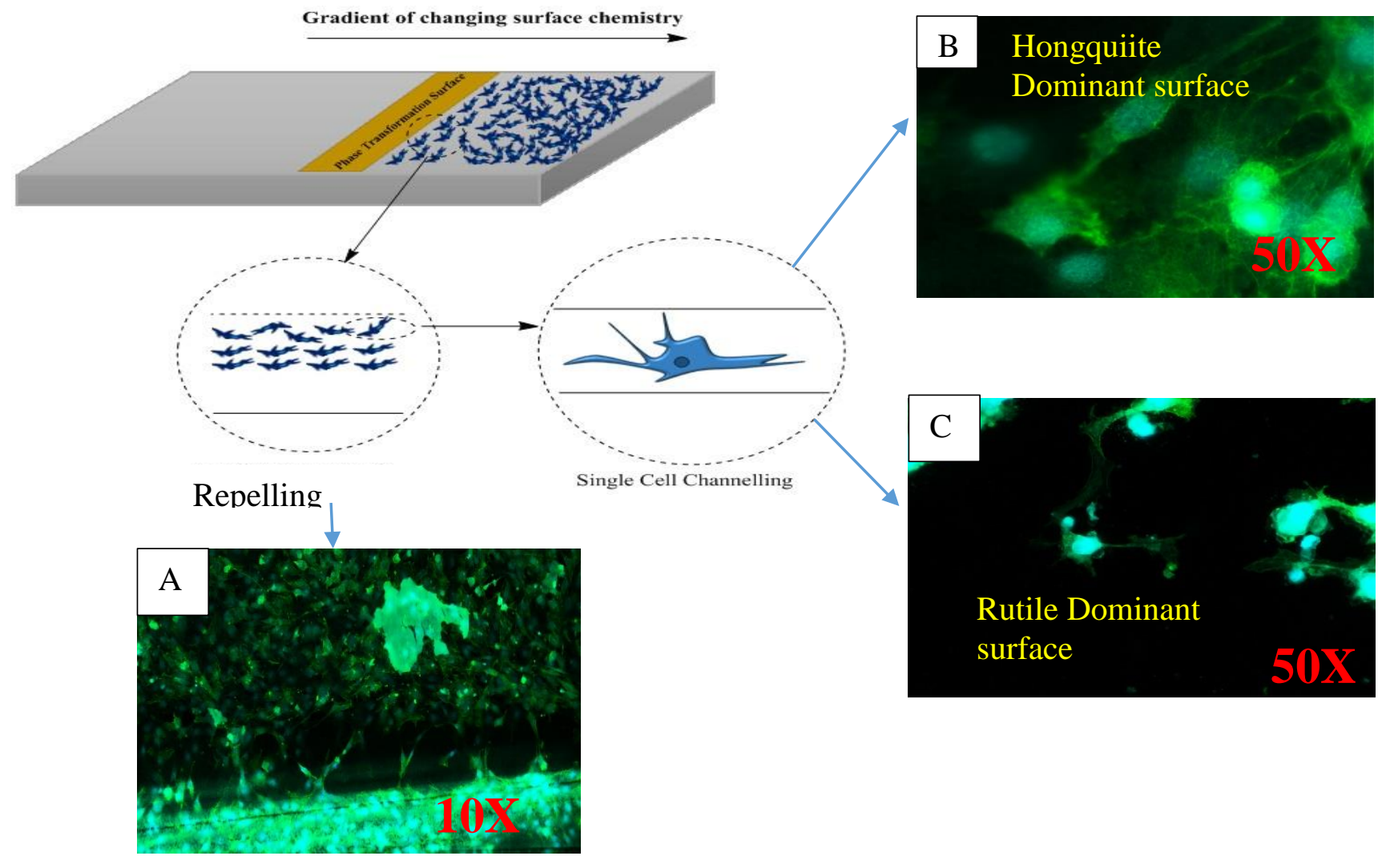

Figure 4-10: Illustration of reduction in cell proliferation and single cell channelling by respective dominant phase surfaces synthesized 


\subsubsection{Single cell channeling by synthesized phase surfaces}

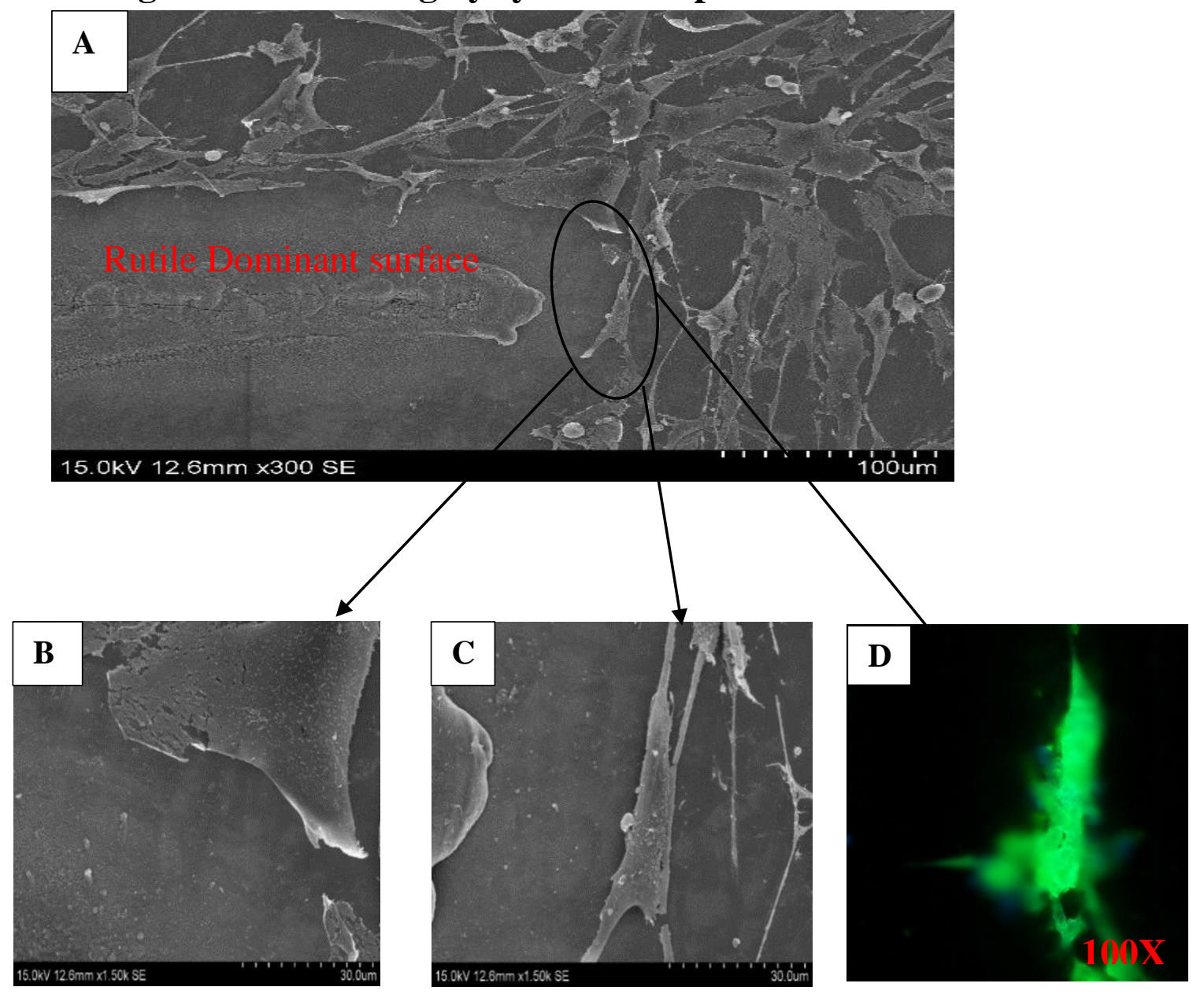

Figure 4-11 (A-D) Single cell channelling on Rutile Dominant Surface with fluorescence images of a single channelled by the rutile dominant phase surface after 48 hours.

As the cells proliferate and come in closer to the rutile dominant surface they bundle up together. From figure4-11 (B-C) insets at closer magnification showing direction and length of the cell travelling away from the rutile dominant surface. Fluorescence microscope image figure 4-11 (D) of a similar single cell moving stretching away from the rutile dominant surface. The bundled up cells look elongated and does seem to have altered its proliferation from flat and uniform to more elongated bundles. These, variations in cell shape were quantified by the area, dispersion, roundness and elongation values. The area, dispersion and the roundness of single cells differed significantly on flat surfaces compared to that of phase dominated surfaces. The cells on phase dominated surface have a smaller projected cell area, cells are round and have a lower dispersion 
compared to cells on flat titanium reference substrate. Thus, cells were smaller and more irregular in shape (stellate), apparently more stretched out (polarised) after the initial 24 hour incubation period.

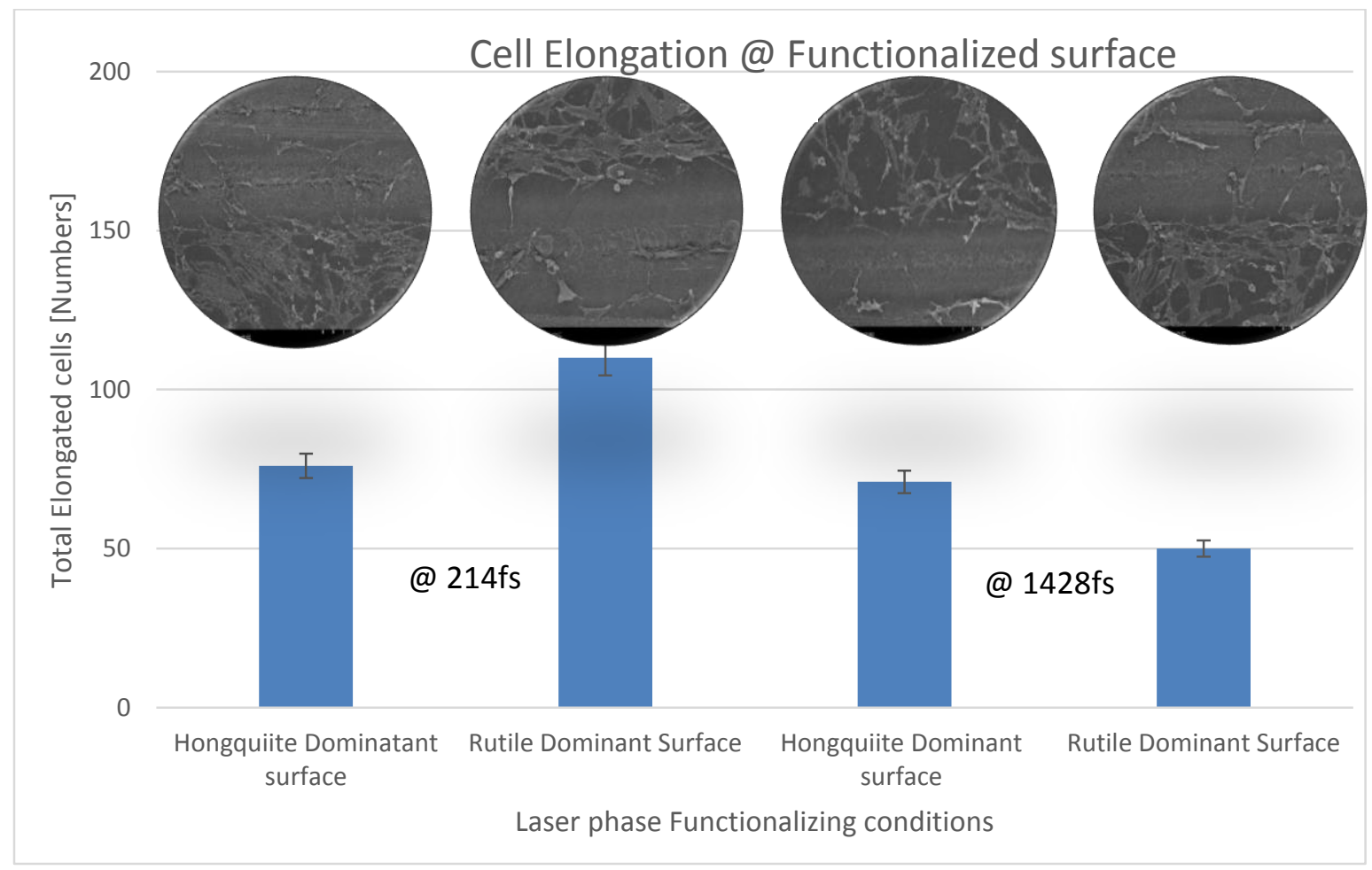

Figure 4-12: Illustrates effect of elongation/ cell streching in osteoblast cell proliferation on different phase dominant surfaces for $\mathbf{4 8}$ hours with varying pulse to pulse seperation time.

After 48 hour incubation the cells have sensed/ probed the laser functionalized surface to find a conducive area to proliferate and differentiate. That conducive area is not on the phase dominant surface where the material surface property is composed of a cocktail of $\mathrm{TiO}, \mathrm{Ti}_{3} \mathrm{O}$ and $\mathrm{TiO}_{2}$ oxides in the respective phases. The above graph explains the degree of elongation of osteoblast cells in terms of number on the phase dominant surface. A trend patterns can also be inferred from the above figure 4-12 where number of total elongated cells near the phase dominant surfaces decreases as pulse to pulse separation time is increased $214 \mathrm{fs}$ to $1428 \mathrm{fs}$. 

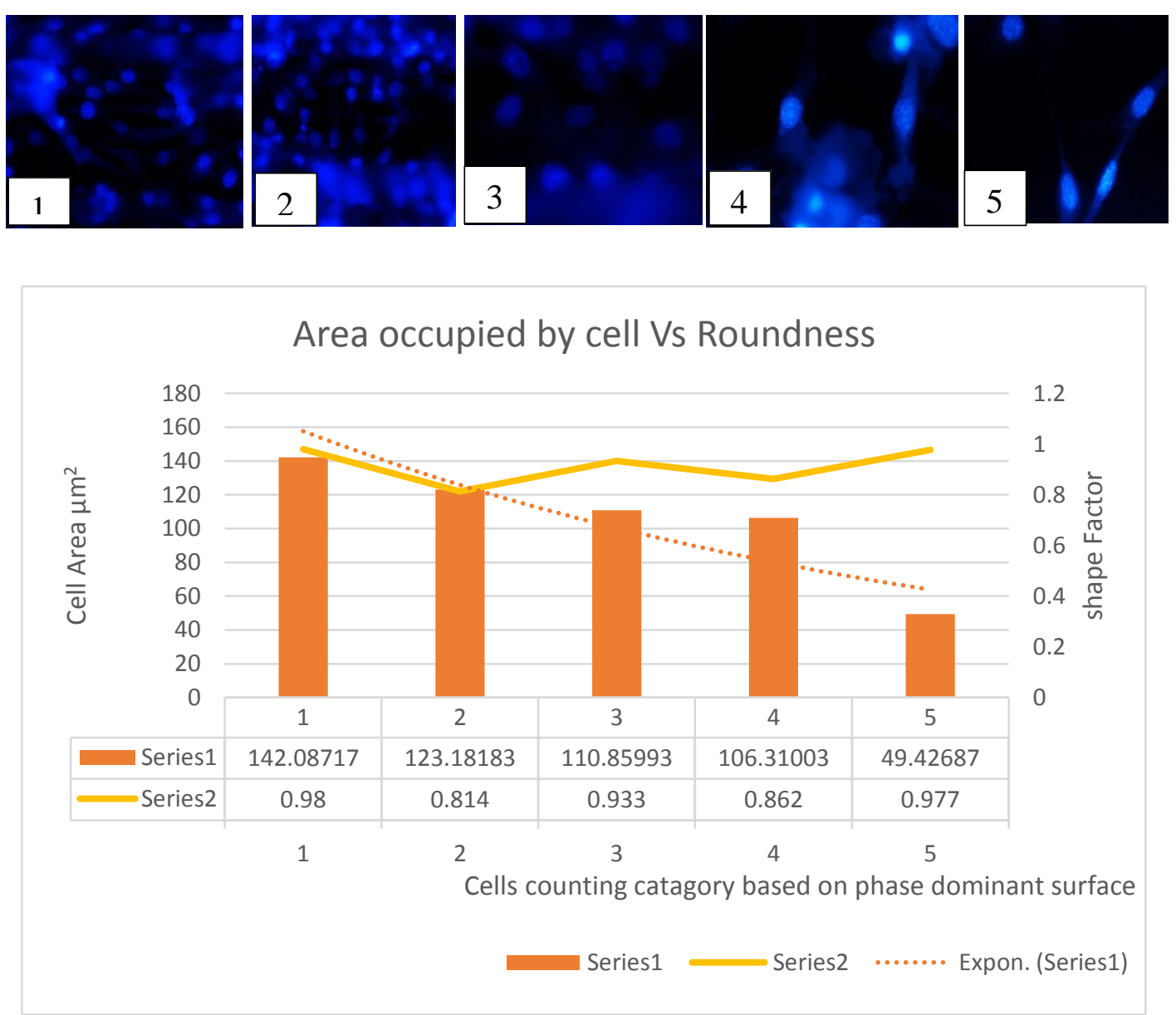

Figure 4-13 : Graph showing variance in area of cell proliferation with roundeness of nuclei In order to understand the activity of cell-cell and cell material interaction certain characteristic analysis tools must be used to first categorize them. Here the activity of each cell is divided into the area it's proliferated and roundness of the nuclei based on the phase dominant surface it proliferated on.The above graph gives a trend line image of cell proliferation activity and nucleus spread near the phase functionalized surface. The area of cell reduces when it come in contact with the functionalized phases surface. A total of five different category of cells were found which had varying range of nuclei spread. A shape factor of 1 is assumed for a perfect circular cell and deviation from circularity is categorized in decreasing order of magnitude. Hence, a nonproliferating cell will have a shape factor close to 1 with low cell proliferation area. Previous results on titanium dioxide rutile nanotube suggest that the nucleus of a cell remains rounded under normal proliferation condition and changes its shape to change in surface chemistry .But, due to complex composition of oxide phases the exact oxide phase percentage that prompts this behaviour 
of both elongation and shrivelled nucleus in the same interacting surface is an interesting phenomenon. Hence as the surface chemistry of the material reaches a critical value of having rutile dominance it decouples certain interacting nucleus making them non-viable for proliferation thus contributing to cell apoptosis.

\subsubsection{Specific cell interactions with rutile dominant surface}

To observe and asses cell viability and behaviour on phase transformed surface towards anisotropic cell development, MC3T3-E1 pre-osteoblast and NIH 3T3 fibroblast cells were made to interact where initial cell attachment/adhesion, proliferation and filopodial activity of each individual cell were studied in detail.
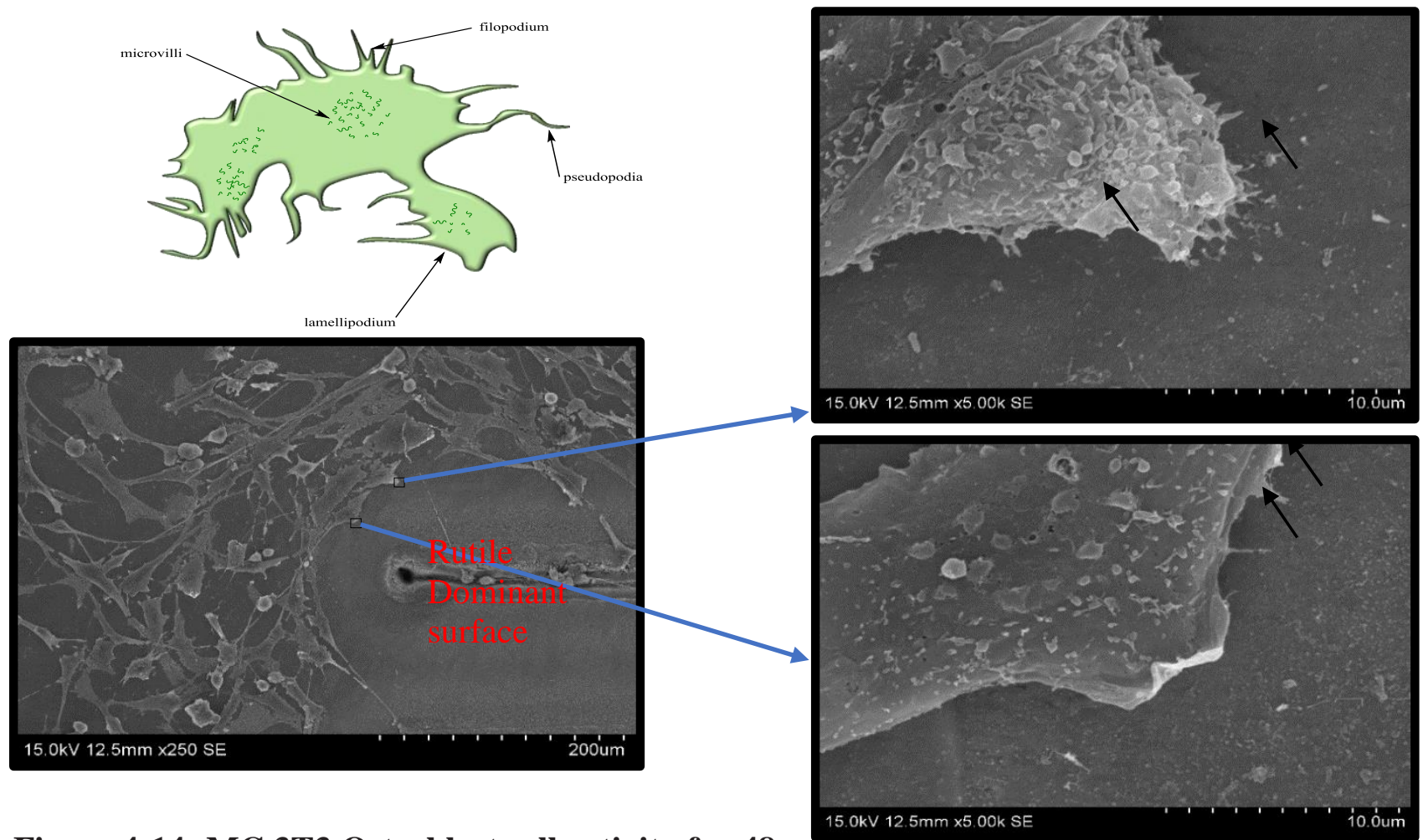

Figure 4-14: MC 3T3 Osteoblast cell activity for 48

hours at Rutile dominant material. A graphic illustration various parts of cell on surface functionalized substrate is represented.The above figure also represents MC 3T3 osteoblast cell activity in control and surface functionalized area

Cell migration is a co-ordinated multistep process involving directed protrusions of the cell membrane, adhesion of the cell membrane to surrounding surface/substrate, contraction of the cell body, and later detachment of the cell at the trailing edge [12]. The growth of cells appear normal 
on the control surface where cells have proliferated over a large area and appear flat with long lamellipodium extensions, indicating a strong proliferation occurrence. But, the cells that are in contact near the phase dominant surface has a different development with far too less lamellipodial extensions. The cells also appear clustered around the rutile phase dominant surface from Figure 4-14 where the surface chemistry is completely different from the control substrate. The magnified images in Figure 4-14 also show the same where almost no filopodial activity is seen on functionalized area. This indicates filopodia have sensed that surface and found it to be nonconducive for proliferation. There are also regions where long lamellipodial extensions are also seen around the rutile phase dominant material strongly suggesting the migration of cells is solely due to change in surface chemistry a physiochemical reaction of the functionalized material.

\subsubsection{Functionalized surface acting as cues for cell repellence:}
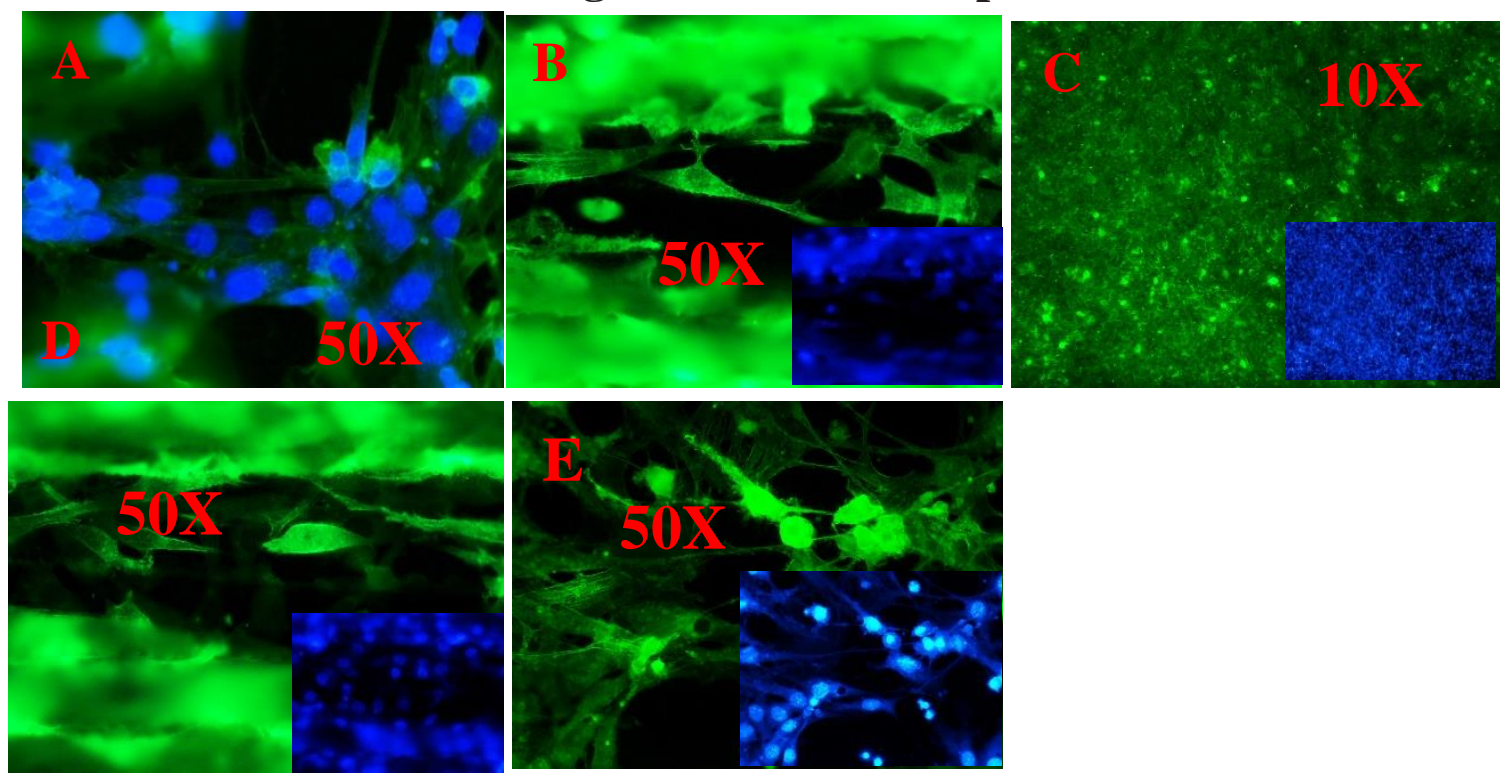

Figure 4-15 Show preferential growth of cells based on phase dominant material. Figure 15 C cell on native titanium surface Fig 4-15 A,B,D\&E are magnified fluorescence data showing the preferential growth alignment with filopodial activity near the oxide phases and cell repellence on the functionalized surface.

From previous literature studies we know osteoblast cells are anisotropic by nature, and their adherent property is highly sensitive to surface structure of material [50-52]. Although the concept of contact guidance was established long time back, the effect of polymorphic titanium oxides phases on anisotropic development was never established. Previous results suggest that the nucleus of a cell remains rounded under normal proliferation condition and changes its shape to change in 
surface chemistry $[53,54]$. The fluorescent microscope data figure 4-15(A-E) reveal the activity of both cytoskeleton and nucleus of MC 3T3 - E1 osteoblast cell on phase transformed surface where, the cell nucleus when comes in contact near the surface change its shape from circular to elliptical definitely suggesting acute change in surface chemistry on the surface. All the cells look elongated showing evidence of cell stretching which leads towards anisotropic migration due to mechanical and surface chemistry cues from the surface in figure 4-15 (A,B,D\&E).The compositional chemistry of the surface which initiated all this process is found to have $(\sim 70 \%)$ cubic TiO, $(\sim 15 \%)$ rutile $\mathrm{TiO}_{2}$ and ( 10 to $\left.15 \%\right) \mathrm{Ti}_{3} \mathrm{O}$ oxide. Cell repellence on a biomaterial is a nascent concept with huge potential application in current short term biomedical fixtures like screws, nuts, bolts and plates where the growth of osteoblast cells can be controlled [55-57].

\subsubsection{Influence of fibroblast cells on functionalized phases}
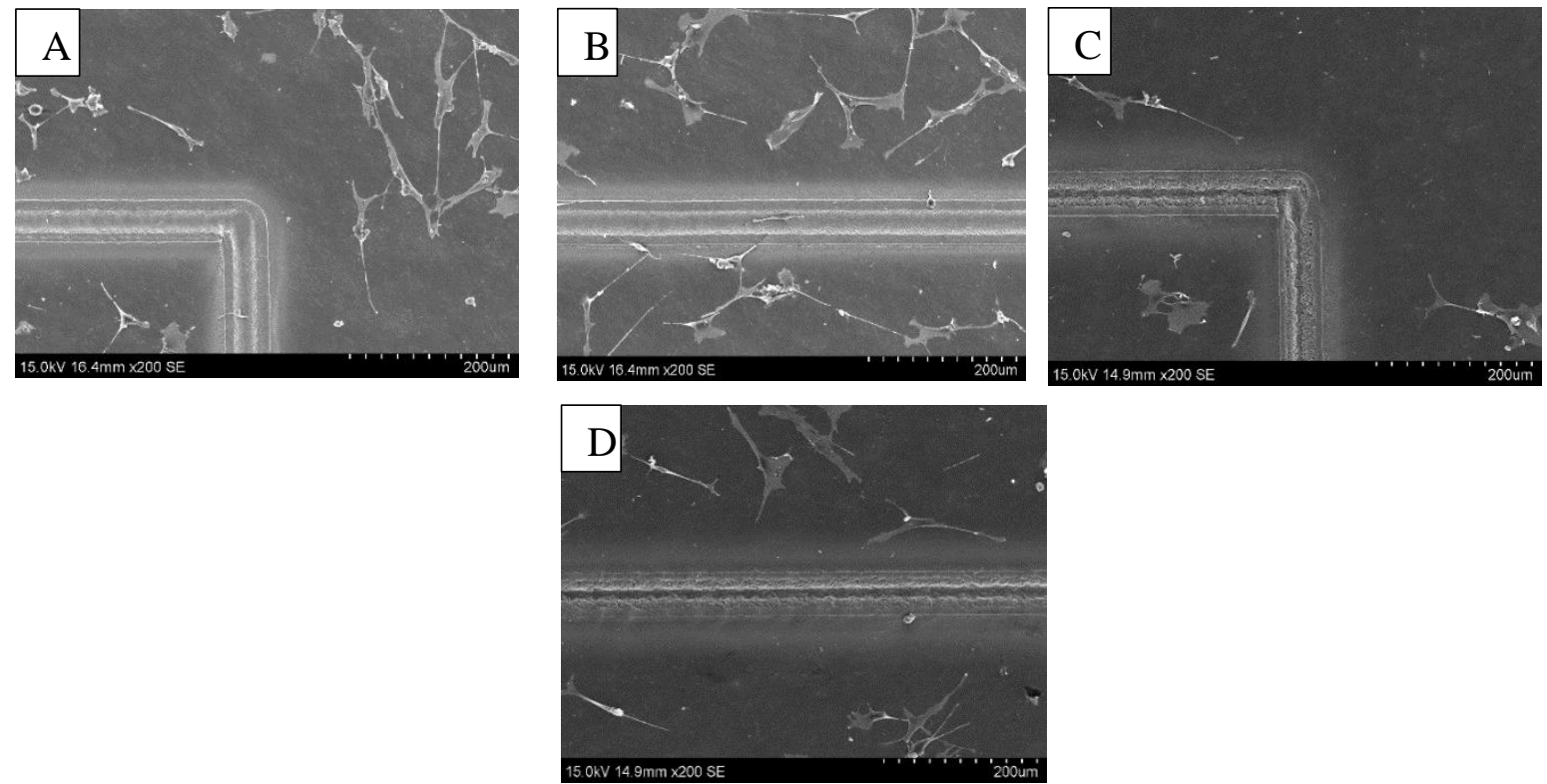

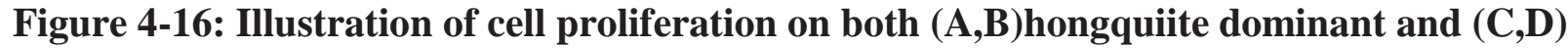
rutile dominant phase surface on NIH 3 T3 cells for 24 hours

NIH 3T3 fibroblast cells when cultured on phase dominant surface demonstrated clear morphological changes, as compared to the flat control surface as shown in figures 4-16(A)-(D) similar to MC3T3-E1 osteoblast cells. NIH 3T3 cells appear elongated in shape and more aligned near the phase transformed surface where the dominance of one phase supersedes the other. This is consistent with all other literature studies showing that both topography and oxide surface can induces adherent cells to elongate and align $[52,53,54]$. The above SEM figures also shows that 
each NIH 3T3 cell although seem spread, the observed elongation and alignment were definitely not a result of physical entrenchment of the cells by the grooves but a more surface oriented reaction between the substrate and cell. However, on all phase dominant surfaces in figure 416(A)-(D) showed cell movement was clearly guided by functionalized oxide phase surface and evidence low cell population closer to functionalized surface was also seen.

\subsubsection{Influence of fibroblast cells on hongquiite dominant phase}

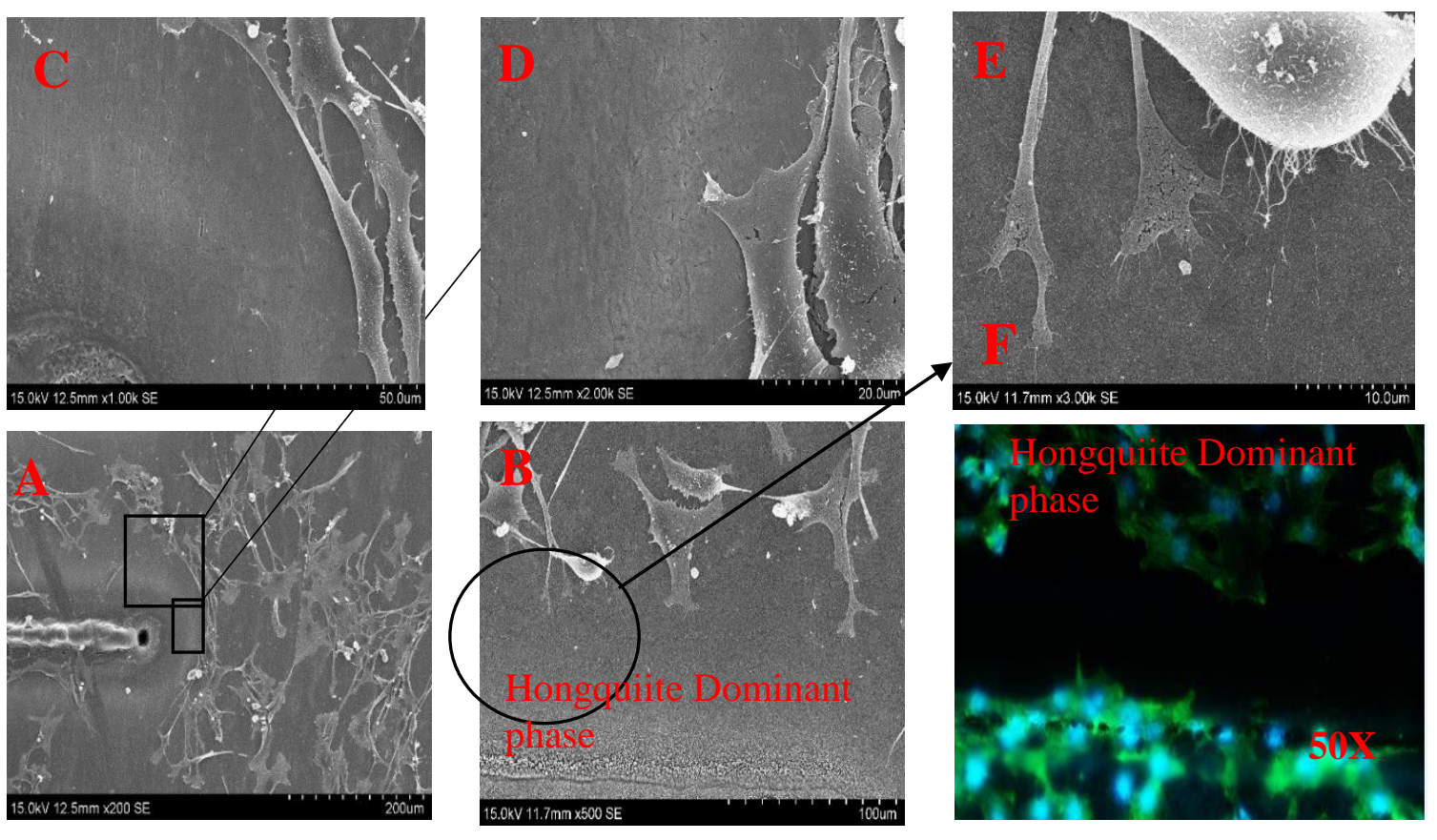

Figure 4-17 A-E: SEM micrographs of NIH-3T3 fibroblast cell for 24 hours. Fig 17 F fluorescent microscope data of the hongquiite phase dominant surface for 24 hours incubation

The above figure 4-17 represent the activity of NIH 3 T3 fibroblast cells on polymorphic titanium oxides phases synthesized having hongquiite dominant surface showed its effect by altering and aligning with initial fibroblast proliferation as seen in above figure 4-17 (A-E).The SEM images in figure 4-17(A) show evidence of cell clustering on control surface similar to MC3T3-E1 osteoblast cells and acute shape change of fibroblast cell near the synthesized dominant phase surface. The magnified micrographs of figure 4-17 (B, C) also confirms the same where evidence of stretching and almost no/low filopodial activity is seen. The results composed concurs with MC3T3-E1 osteoblast cell activity where anisotropic cell development was stressed from Figure 4-15. Figure 4-17(D, E) show fibroblast filopodial sensing on the polymorphic titanium oxide 
surface synthesized not viable for proliferation. The small fiber like structure protruding out from the cell called as short filopodia which aid in sensing and determining the optimum surface for proliferation stopped its proliferation just before the synthesized oxide surface. The long thick lamellopodia of the cells assisted in migration away the phase dominant surface. The surface analysed here has initiated the NIH 3T3 fibroblast cell to start a series of filopodial cues to sense the direction conducive enough to migrate. Fluorescent microscope data in figure 4-17 F confirms the above explanation where cell clustering and evidence of anisotropic cell movement is witnessed.
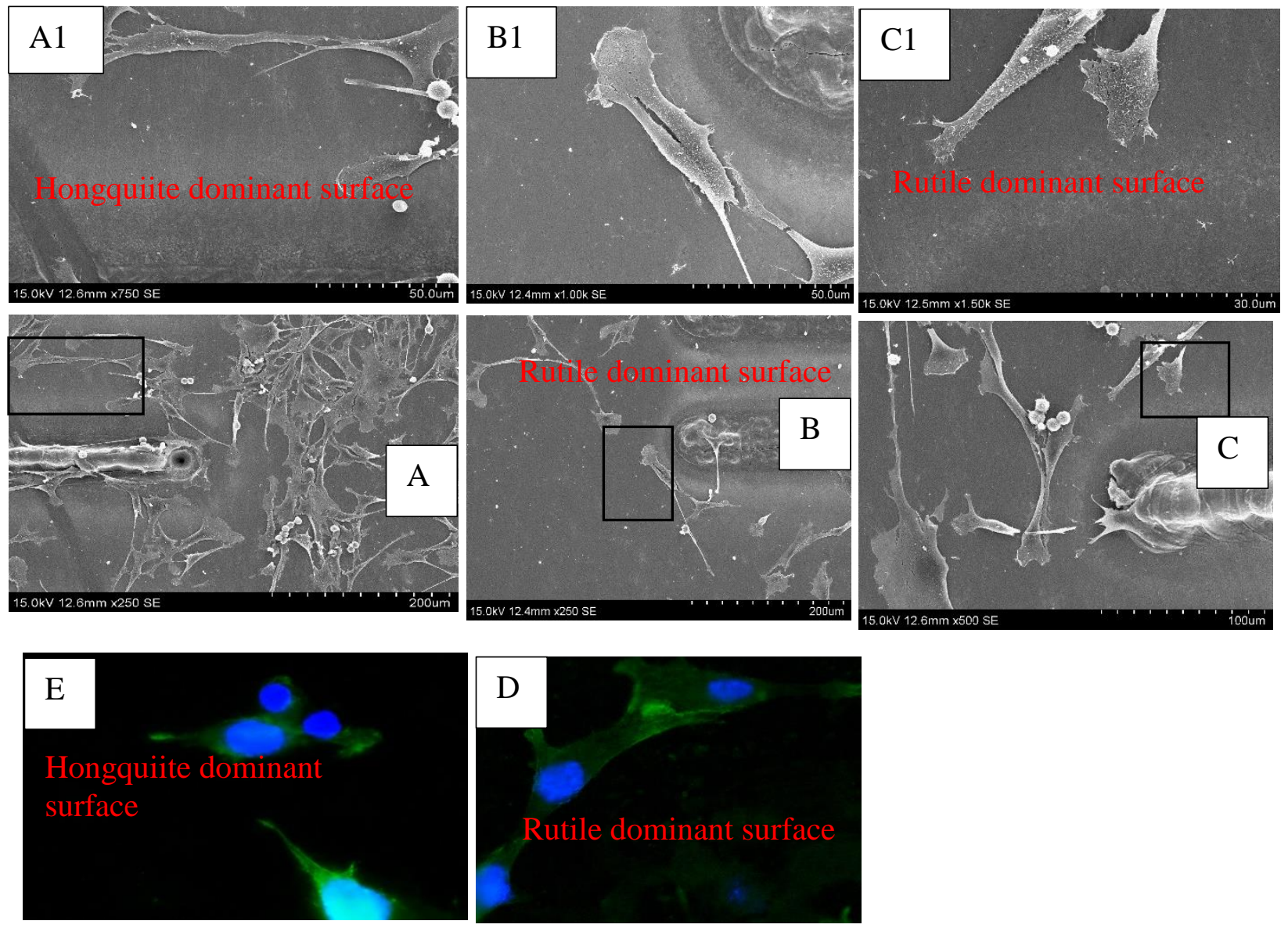

Figure 4-18 A-E: SEM analysis / Fluorescence microscope data of NIH-3T3 fibroblast cell for 48 hours on both hongquiite and rutile dominant phase surface. A1-C1 are magnified images showing individual cell activity on phase dominant surface

As the above SEM micrographs clearly details the sequence of activities taking place on the phase dominant material. As the surface compositional chemistry was altered from hongquiite dominant to rutile dominant phase surface figure 4-18 (A-C) the fibroblast cells elucidated specific 
responses from directional alignment with higher proliferation rate on hongquiite dominant surface figure 4-18(A) to low cell proliferation control rutile dominant on figure 4-18(C). The fluorescence data also show evidence of directional alignment and migration of NIH 3T3 cells with changing nuclei shape and arrangement very similar to MC3T3-E1 osteoblast cell.

\subsection{Summary}

It is very essential that connective tissue cell growth on implant surface be avoided at all costs as they hinder to proper fixation of implant to bone cells. The above results proves with absolute certainty that phase composition of the synthesized surface having a unique surface chemistry can instigate a specific response from cell although having same morphological characteristics. The above results clearly suggest an unforeseen way for controlling cell population on titanium implant surface by accurately programming the surface oxide phase composition thereby creating localized functionalization through USP laser phase transformation technique. Thus altering and repelling cells on a surface that stops growth of connective tissue but promotes osteoblast cells can now be harnessed as an asset for hard implants. 


\section{Chapter 5 - Non catalytic approach of nano-core structure and cell sustainability}

\subsection{Introduction}

This chapter demonstrates the novel non-catalytic fabrication of core-shell like nanostructures on a titanium substrate. Here the formation mechanism of this core-shell like nanostructure is analysed during USP laser irradiation in atmospheric conditions. The synthesized nanostructure was then made to interact with both primary osteoblast and fibroblast cells to examine the interaction of synthesized nanostructure on cell proliferation and adhesion. The versatility of the smart interactive nanostructure synthesized can be applied to drug delivery for targeted actions and biomaterial implants.

\subsection{Generating mechanism of engineered titanium nanostructure}

Several kinds of species are generated during ultra-short pulsed laser interaction where the very short plume expansion makes it impossible to determine the exact kinetic reaction. Two common reasons are responsible for complex phase coexistence inside the plume are first, spatial intensity and secondly condensation reaction [41]. Due to the increased laser intensity structural phase transitions give way leading to melting, ionization and ablation at the target surface, as a result leading to non-homogeneous vapor condensation of the plume. Although, total atomisation in the plume by ultra-short laser was proved earlier in materials like silicon [44] and copper their effect is not fully matriculated for biomaterial like titanium [48]. There exist an inconsistent heating volume inside the plume where the plume temperature is maximum at the target surface and decreases as the plume rises up. Four stages of reaction is said to take place during vapour condensation reaction. Cluster nucleation, growth of nucleated species, cooling and deposition of reacted species. The species present inside the vapor plasma often interact with the ambient gas leading to aggregation of atoms and molecules forming nanoclusters [58]. 


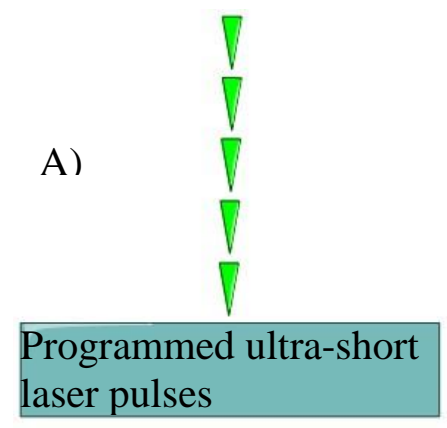

C)

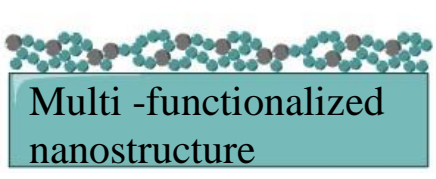

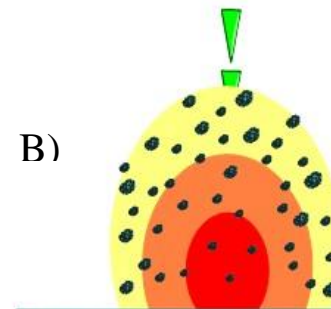

Multi photon ionization by vapour condensation

D)

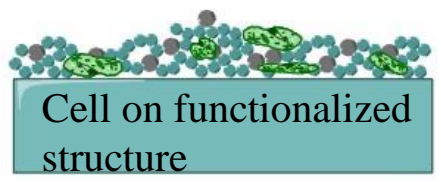

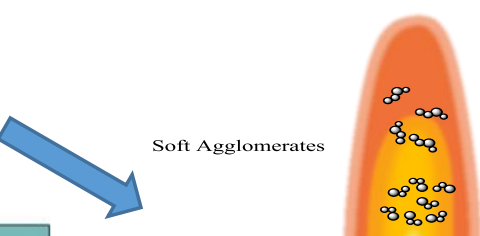

Interacting oxygen in plum

$\circ \circ$

Ionization species
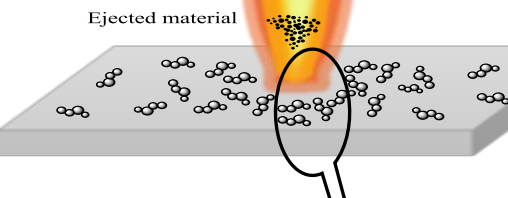

Formation of multi- $\oint$ pre like nanostructure formatijon inside
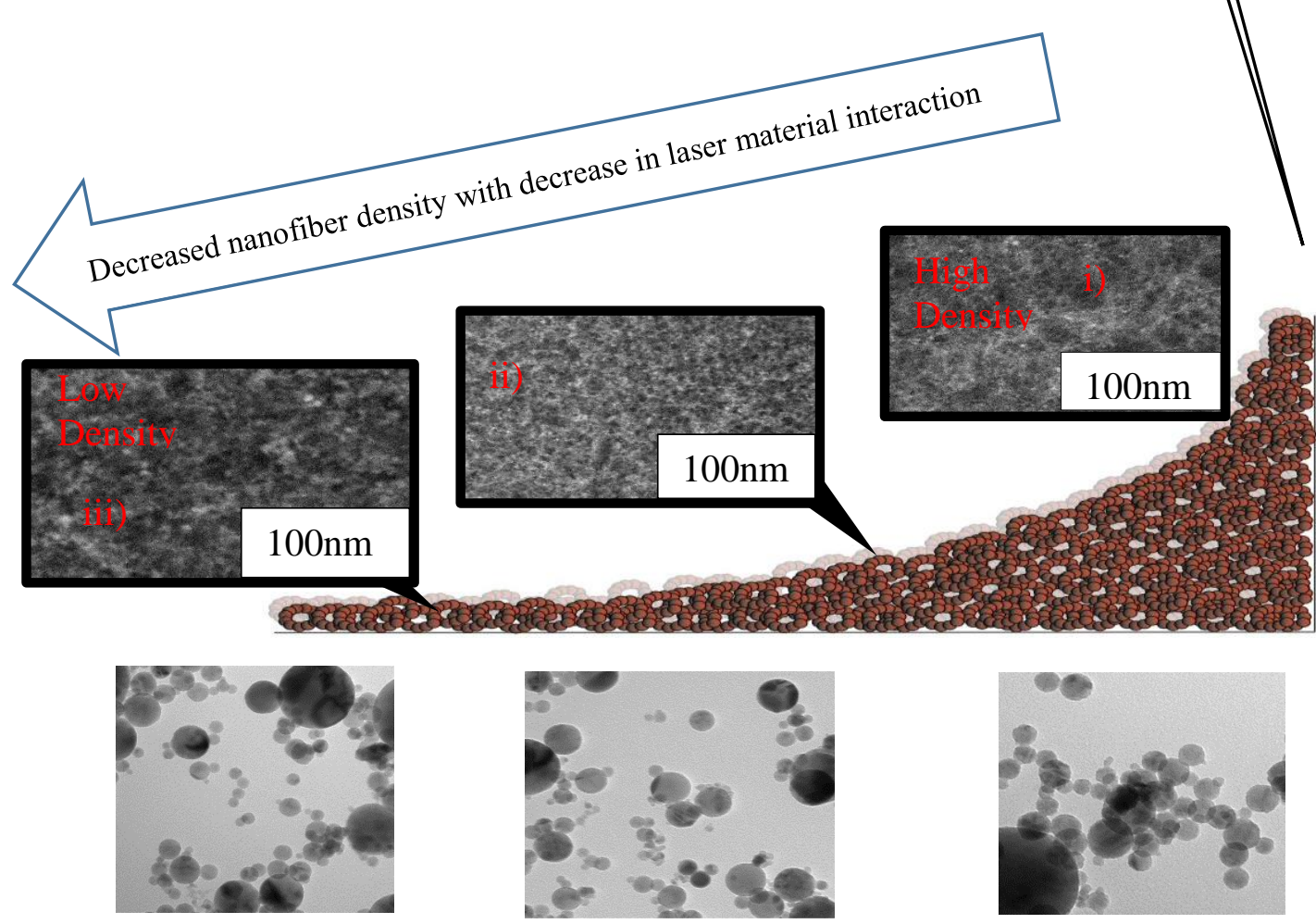

Figure 5-1: Illustration of nanostructure synthesis by vapor condensation mechanism on titanium by USP laser. Graphical representation of nanostructure density with SEM magnification. i) Appearance of the synthesized nanostructure at high density site; ii) organization of self-assembled highly inter connected nanofibrous structure iii) strands containing individual nanoparticle assembled to form a core shell like nanostructure (A-C) respective nanoparticle size variation with peak power. 
The above SEM micrographs in figure 5-1 nanostructured material show lightly packed yet highly interconnected nanofiber. The porous appearance of the laser treated surface is a result of loose self-assembled nanofibrous strands with diameters varying from hundreds of nanometers to few micrometers depending on laser material interaction conditions. Although the change in density of the synthesized Titanium oxide nanostructures by USP laser with laser pulse frequency was previously established by Tavangar et al., the above fabricated structure here is unique as the both amount of oxidation and phases in the oxides can be individually controlled by USP laser through vapor condensation mechanism. The synthesized nanostructure regions were then observed under EDX primarily to confirm the presence of titanium and oxygen nanoparticles in the structure in figure 5-2.

\subsection{Elemental composition of the novel nanostructure synthesized}

The structure and density of the nanofibrous architecture synthesized is influenced by various laser parameters, such as laser fluence, laser pulse frequency rate and laser pulse to pulse separation time. Here, the influence of laser pulse frequency rate on nanostructure density, and size of the generated nanoparticles in the nanofibers was investigated. Native Titanium surface was irradiated at different laser pulse frequency rates from $4 \mathrm{MHz}$ to $12 \mathrm{MHz}$ at a constant laser pulse irradiation time of $5 \mathrm{~mm} / \mathrm{s}$. Figure 5.1 SEM images of titanium nanofibrous structures generated over a Titanium substrate at low laser pulse frequency of a) $4 \mathrm{MHz}$ and b) $12 \mathrm{MHz}$. A detailed view of the structure shows that it consisted of self-assembled interconnecting rings where nanoparticles are aggregated together. 

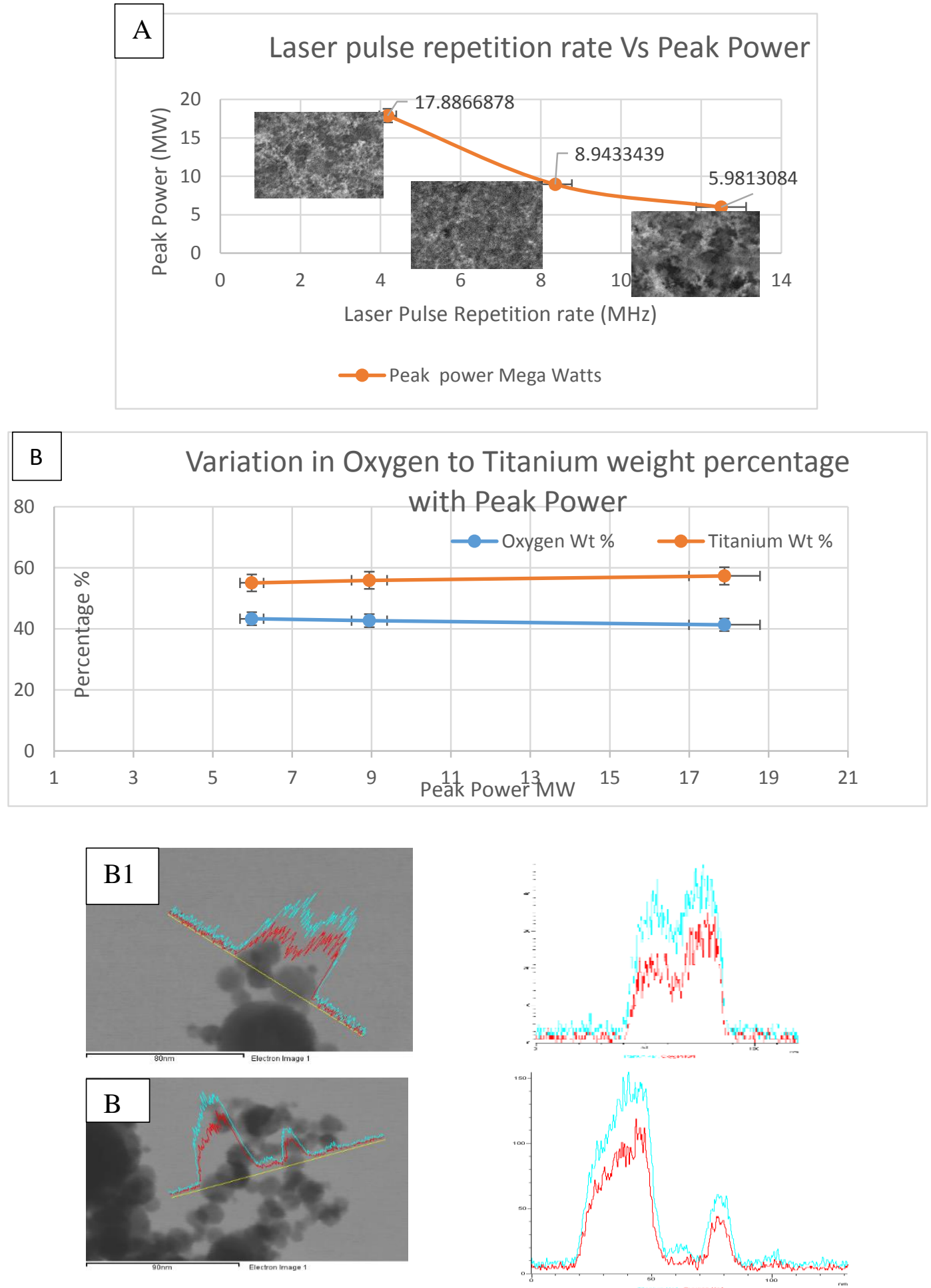

Figure 5-2: Show synthesized nanostructure variation with laser peak power and laser pulse repetition rate (B) Effect of increasing peak power on core shell like nanostructure formation (B1-B2) TEM/EDX intensity data indicating variation of titanium to oxygen ratios at low and high laser peak power conditions 
The above TEM/EDX figure shows the individual elemental composition of both Titanium and oxygen in the observed nanoparticle. The TEM/EDX data mapped above indicates that $\mathrm{O}$ and $\mathrm{Ti}$ are homogenously distributed throughout the observed area show spread of nanofibrous structure. When comparing the intensity of Titanium to oxygen figure 5-2(B1-B2) at low and high laser peak power, a clear distinguishable pattern is available signifying a more densely packed very uniform spread of nanoparticles if found at high peak power signifying more oxidation. A similar kind of trend pattern can be inferred form oxygen compositional variation from figure 5-2 (B1-B2) where intensity of oxygen dips at the intermediate space between nanoparticles and forming a core like shell over the synthesized titanium species. This oxygen is most probably $\mathrm{TiO}_{2}$ forming a passive layer over each nanoparticle synthesized.

\subsection{Characterization of synthesized nanostructure}
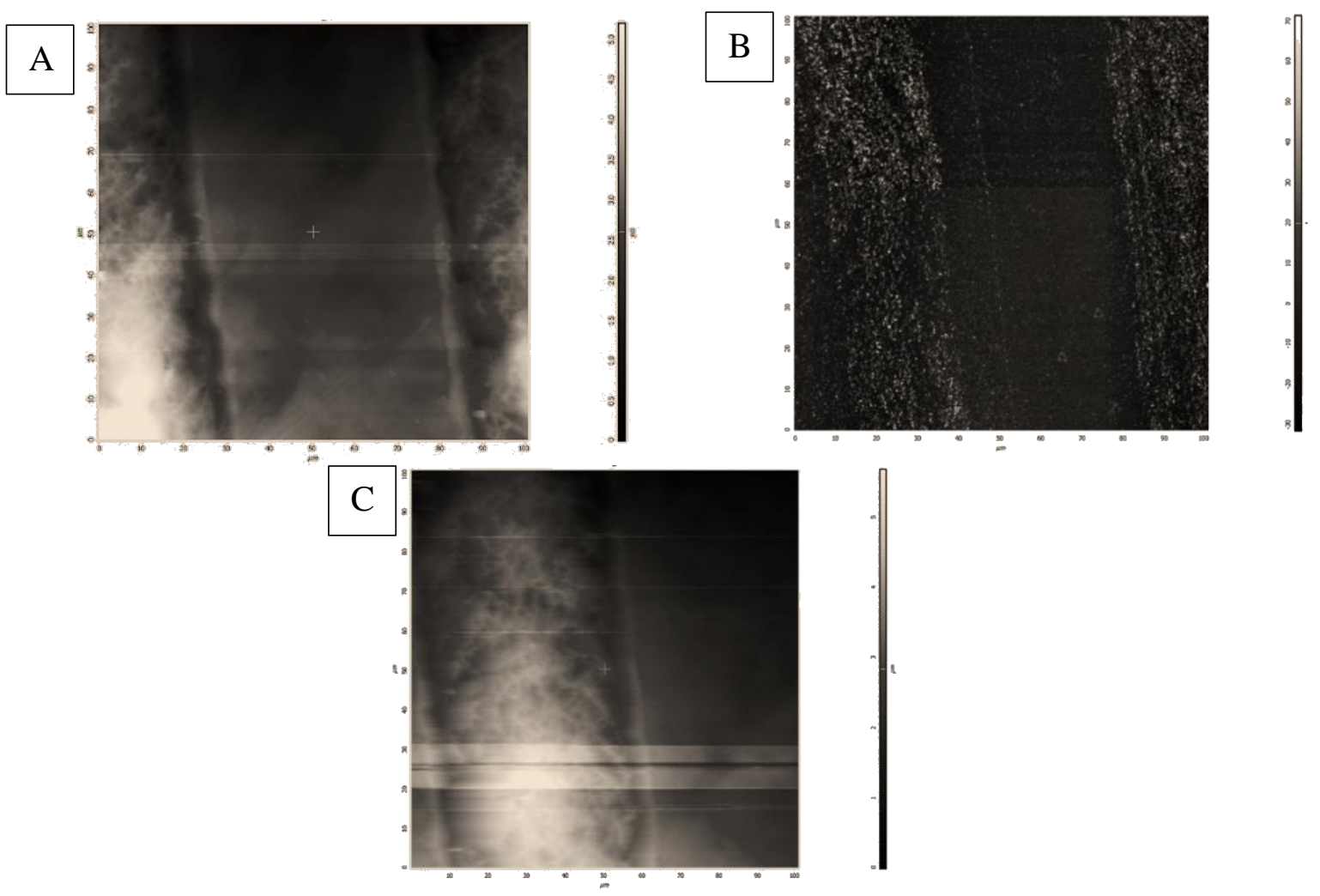

Figure 5.3: A-C AFM Scans of nanostructured titanium substrate. Figure 3B phase activity on the nanostructure. Figure $3 \mathrm{~A}$ height scan data at high laser pulse frequency rate(25 $\mathrm{MHz})$ and figure $3 \mathrm{C}$ is height scans at low laser pulse frequency rate( $4 \mathrm{MHz}$ ).

The above data shows change in material characteristics with non-uniform cloudy structure from the normal surface. The native titanium substrate has a relatively smooth morphology with no 
significant changes in the surface. But, both material property and morphology seems to change in nanostructured zone from at high laser pulse frequency/ low peak power $(25 \mathrm{MHz})$ which is in correspondence with EDX data from figure 5-2 (B). A pattern of non-uniform cloudy structure is observed figure 5-3(A,C) during the scans which first implies the presence of nanostructure and a complex mixture of oxides phases of varying intensities from the non-uniformity of the observed structure. The depth saturation suggest prolonged laser irradiation time (i.e. scanning speed) with dense nanofibrous structure. Then, X-ray diffraction was used to analyse, quantify and investigate the effect of nano crystalline phases synthesized in the nanostructure by USP laser.

\subsection{Oxide phase composition of the synthesized nanostructure:}

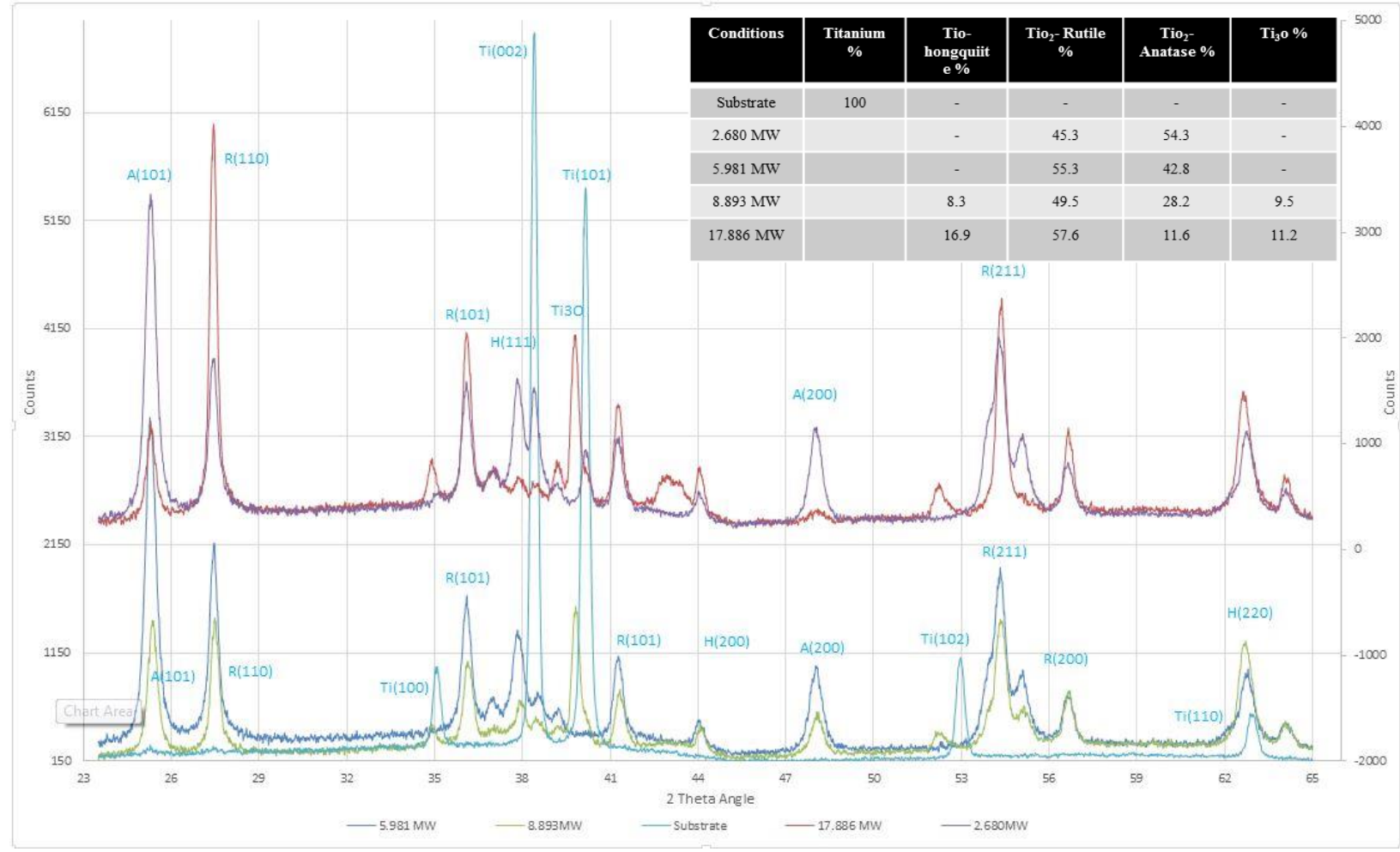

Figure 5-4: XRD Micrographs illustrates varying laser peak power on material interactions for synthesis of titanium core shell like nanostructures. A constant pulse width of (214 fs) and (1428fs) was maintained to measure change in intensity of oxide phases synthesized.

With regard to the crystalline structure in the nanostructure the above XRD graphs Figure 5-4 indicate that all the samples exhibited a polycrystalline structure and consisted of anatase [2] and rutile. [3] Titanium dioxide is considered as the one of the thermodynamically stable oxides. [4] The synthesized nanostructure here shows the presence of two thermodynamically stable phases 
of Ti-oxides anatase and rutile with domination of anatase especially at 5.98 MW laser peak power. The dominant phase component became rutile, while anatase is reduced to $8-12 \%$ wt at 8.963 MW. This sudden shift in oxide phase dominance is brought out by increased laser pulse to pulse separation time from $214 \mathrm{fs}$ to 1428 fs when more laser energy gets transmitted into the material thus resulting in increased synthesis of high temperature phase rutile. However, the dominant oxide phase still remains anatase occupying nearly $\sim 55 \%$. The composition takes a sharp change at 17.886 MW where aside from anatase and rutile, 2 more Ti-oxides appear, trigonal $\mathrm{Ti}_{3} \mathrm{O}$ oxide and a rare non-stoichiometric cubic $\mathrm{TiO}$ oxide having hongquiite phase. The amount of the 2 new Tioxides varied from $\mathrm{Ti}_{3} \mathrm{O}(15-17 \%) \mathrm{TiO}(28-30 \%) \mathrm{wt}$. The probable reason for synthesis of these additional 2 new oxide apart from anatase and rutile phases is effect of peak power generated from each laser pulse at low pulse frequency. The peak power generated here is high compared to high laser pulse frequency as only few pulses holding intense power hits the target surface. In all xrdpatterns peaks from the Ti-substrate can be detected. This is a clear evidence that the whole thickness of the Ti-oxide layers at all conditions were fully penetrated by incident $\mathrm{x}$-rays. The calculated depth of penetration of x-ray for a composition of $60 \%$ anatase and $40 \%$ rutile is within the range of $5-15 \mu \mathrm{m}$ for the scanned range of $25-65$ degrees 2-theta. Hence, a new class of engineered nanostructure having tunable oxide phase characters which has potential towards cell manipulation is developed using USP laser synthesis.

The surface temperature at the laser material interacting surface was calculated for silicon by from the below equation 5-1. The same can be inferred for titanium in order to estimate average surface temperature at the target surface [59].

$$
T=\sqrt{\frac{2 a}{\pi^{3} t_{p}}} \frac{4 K(1-R) P}{k f d^{2}}
$$

Where $\mathbf{t}_{\mathbf{p}}$ is pulse to pulse separation time, $\mathbf{a}$ is the thermal diffusion co-efficient, $\mathbf{k}$ is the heat conduction coefficient, $\mathbf{R}$ is the reflection coefficient, $\mathrm{K}$ is the residual energy co-efficient, $\mathbf{d}$ is the laser spot size and $\mathrm{f}$ is laser pulse frequency.

Table A.1 (Supplementary information) enclosed present the temperature synthesized on the material surface after USP laser irradiation on titanium.

This inconsistent heating helps in releasing oxygen ions into the plume where they combine with ejected titanium ions species swirling inside the plume's partial pressure variation. The combinations of these ions thus lead to formation to multitude of oxides phase like $\left(\mathrm{TiO}, \mathrm{Ti}_{3} \mathrm{O}\right.$, 
$\mathrm{TiO}_{2}$ ). These oxides species condense and cool to form self-assembled nanofiber like structure. Change in laser fluence helps in realizing a diverse range of nanostructure with varying density and quality and quality of oxide phase synthesized. The engineered nanostructures are distinctive of its kind as the mixture with the specific composition was never synthesized before. It is also evident from the XRD analysis in figure 5-4 that laser pulse frequency help in determining the dominant oxide phases and laser pulse width aids in intensity of the synthesized oxides.

\subsection{Characteristics of Engineered nanoparticles in the nanofibrous structure}

From equation 5-1 the surface temperature during laser material interaction with peak power can be predicted. In order to study the effect of engineered oxide phases and degree of nanoparticle aggregation TEM micrograph in figure 5-5 showed the average diameter of each nanoparticle generated in anatase phase dominant nanostructure.
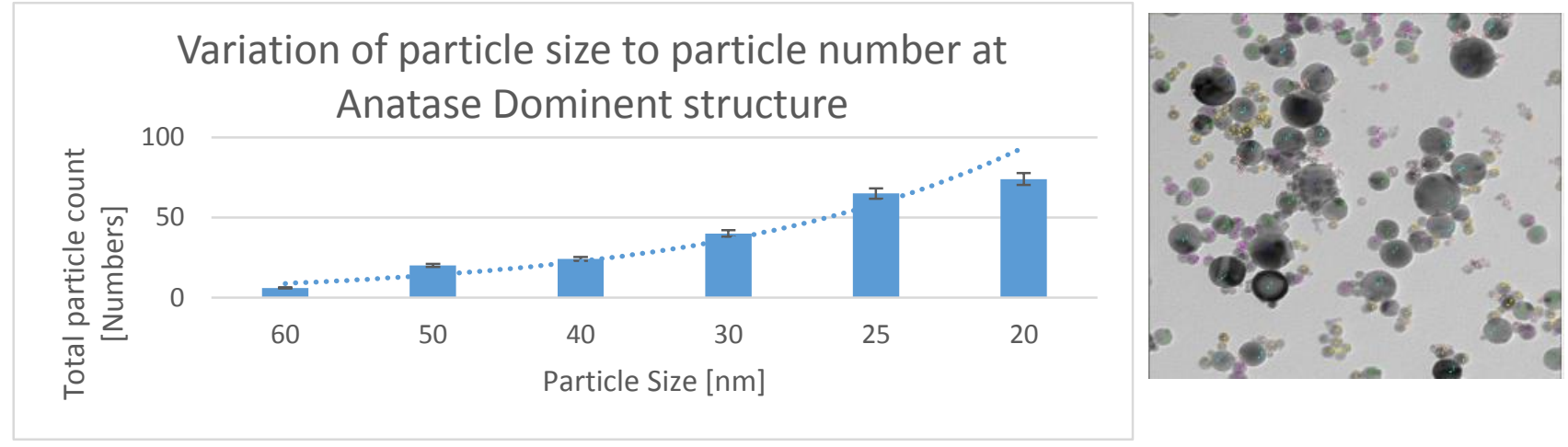

Figure 5-5: TEM image and particle size variation of synthesized anatase phase dominant structure

Titanium and its oxides are generally spherical structures and highly dispersed in size and phase with the Titanium particles being walled by oxygen particles as seen from TEM/EDX figure (5-2 B1). The diameter of each particle was analysed using imageJ software to determine a relationship between particle sizes synthesized phases. The average diameter of particle synthesized here varied from 60- $20 \mathrm{~nm}$ with $20 \mathrm{~nm}$ particles found in excess comparing with others. 


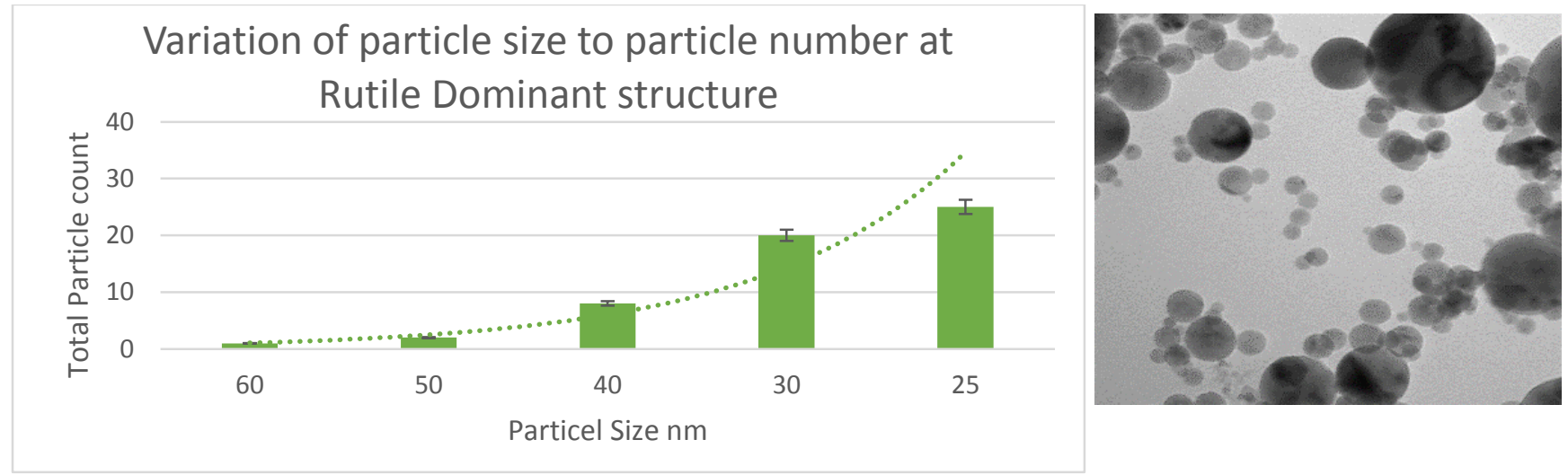

Figure 5-6: TEM image and particle size variation of synthesized Rutile phase dominant structure

Here both laser peak power and width of laser pulse (214 to $1428 \mathrm{fs}$ ) is increased resulting in more oxidation and thus leading to high temperature oxide phase structure from XRD analysis figure 53. But, an interesting phenomenon is witnessed where total particle count is dramatically reduced comparing with figure 5-4 anatase phase dominant structure having smallest particle size observed to be $25 \mathrm{~nm}$. Hence, this low particle count can be directly attributed to increased laser peak power and laser pulse width of $1428 \mathrm{fs}$.

\subsection{Engineering cell repelling nanostructure}

The nanostructures synthesized adhere strongly to titanium surface owing to high oxide phase transformation in the interacting plume. Contradicting to all 3D - ECM structures, the above synthesized nanofibrous structure see consistent reduction in cell proliferation with increasing nanostructure density. Eliminating mammalian cell adhesion and survival on nanowire array was explored by Brammer et al., for biomedical applications like drug delivery, biosensors and blood contacting applications. [60] Anchorage dependent cells like osteoblast and fibroblasts need to attach and spread over a surface for normal proliferation and modulating this has promising applications in biomedical implants $[61,62,63]$.

To study the effect of synthesized nanostructures on cell adhesion, MC3T3-E1 osteoblast cells was studied under a SEM. The above figure 5-7 shows the SEM images of MC3T3-E1 cells where cells on the control surface grew well, indicating that the surface was biocompatible and suitable for cell proliferation. The cells on synthesized nanostructure were mostly rounded and could not spread. However, on the control surface, the cells also appeared flatter and had more extended 
morphologies. The cell attachment and spreading on a biomaterials is always mediated by focal adhesion complex [64]. It has been revealed that the spacing between the integrin ligands is also important for integrin clustering and focal adhesion formation [65-66]. There is more evidence that focal adhesion assembly requires the spacing between ligated integrins to be less than $70 \mathrm{~nm}$. [67]. Distances larger than these reduce clustering which in turn reduces focal adhesion formation. In our nanoparticle study from figure 5-(5,6) the average diameter of each synthesized Nanoparticle in the nanostructure is about 20 to $50 \mathrm{~nm}$, while the distances between nanostructures can vary from few nanometres to several hundred nanometers. Hence, although integrin clustering may occur over the nanostructure, the microscale focal adhesion assembly has impaired because of the long distances between nanostructures.

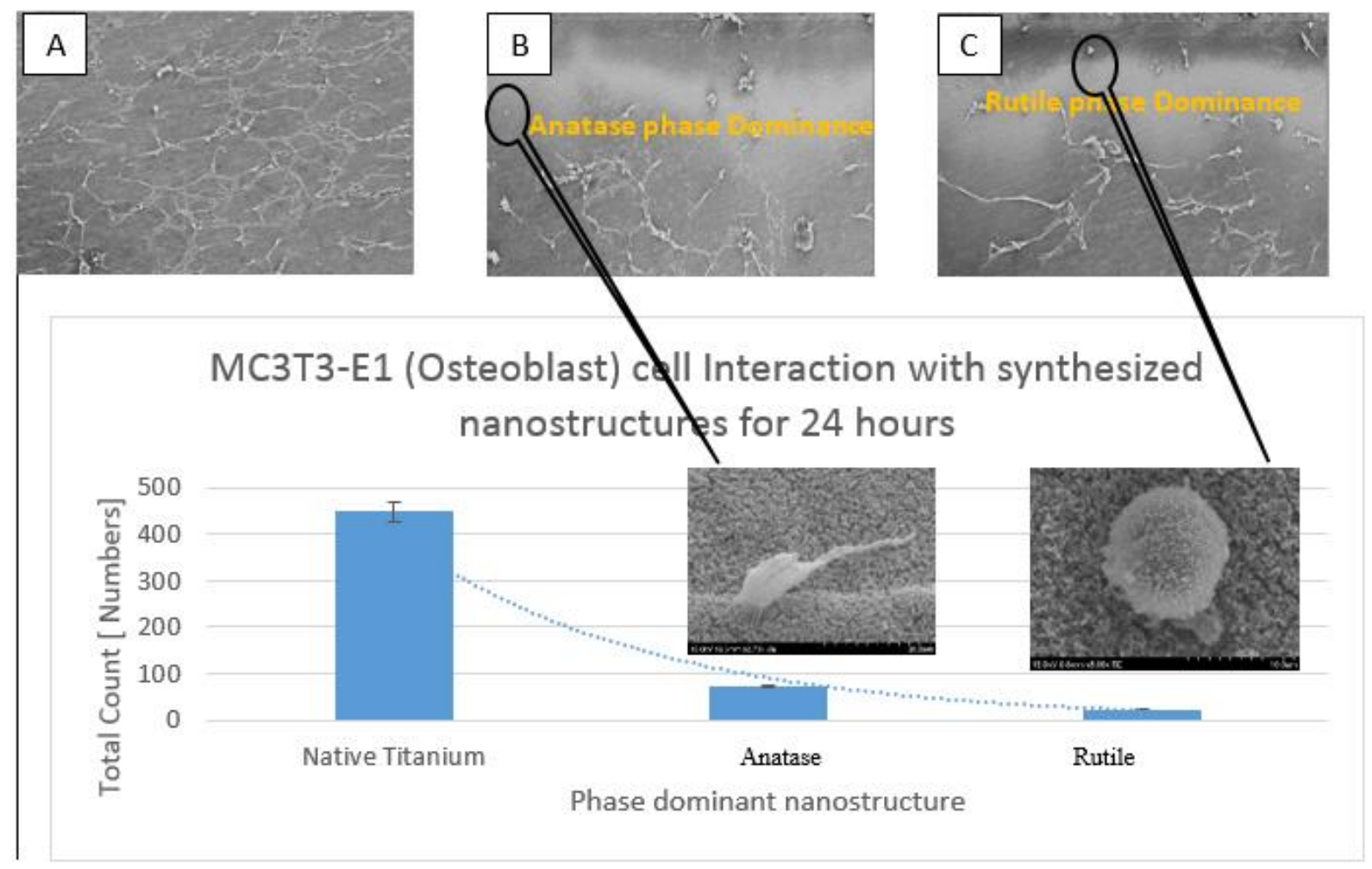

Figure 5-7: SEM micrographs of MC3T3-E1 Osteoblast on native (A) anatase (B) rutile (C) phase dominance after 24 hours 

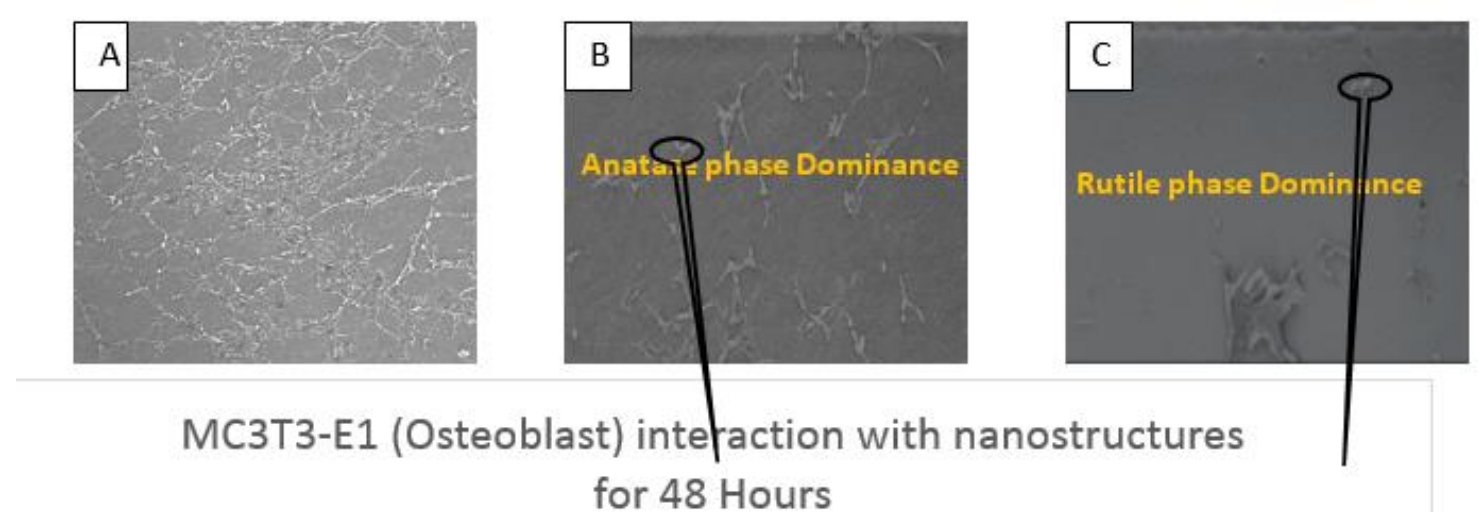

MC3T3-E1 (Osteoblast) interaction with nanostructures for 48 Hours

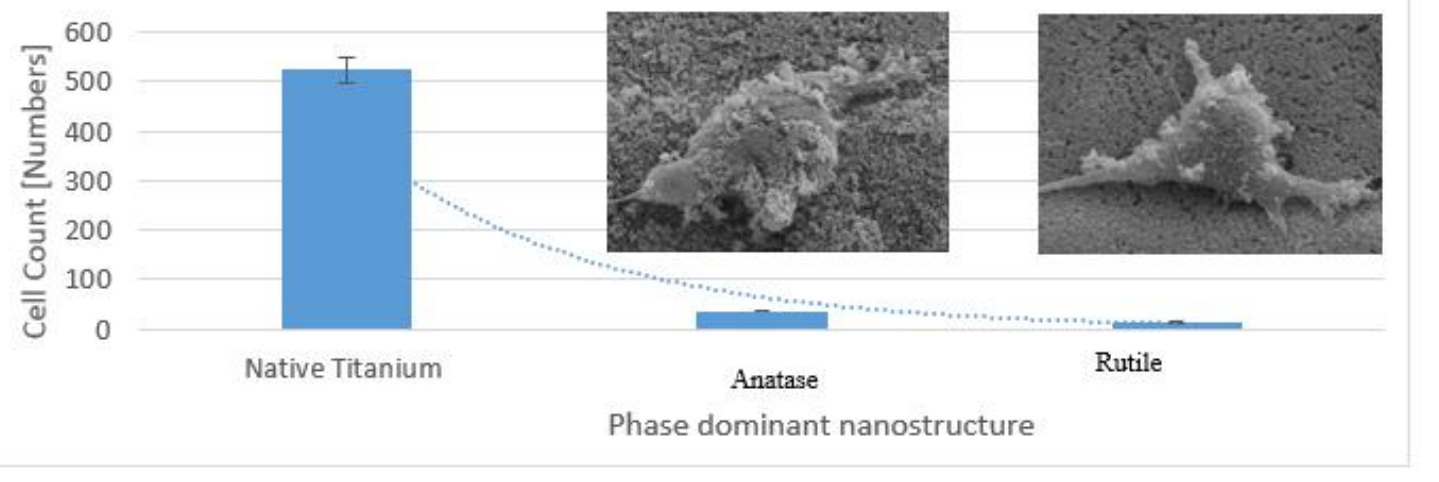

Figure 5-8: SEM images of MC3T3-E1 Osteoblast on native (A) anatase (B) rutile (C) phase dominance after 48 hours

It is evident from the above Figure 5-(7,8) that there is approximately a $96 \%$ i.e ( 19 times) reduction in cell growth in terms of population on rutile phase dominant nanostructures fabricated at high peak power. Native titanium substrate is taken as the control sample. There is more rounded cells present on rutile phase dominant nanostructure region where there is cell entrapment by the nanostructures. It is observed after 24 hours where more cells seem to attach on anatase phase dominant nanostructures when comparing with rutile phase dominant nanostructure figure 5.7. But, when the cell incubation time was doubled to 48 hours osteoblast cell seem to get rounded at rutile phase dominant nanostructure suggesting cell repellence. Since osteoblast cells are attachment sense oriented and providing a non-conducive environment for proliferation may ultimately yield to apoptosis. Also, the cells observed in anatase phase dominant nanostructure show different characteristic growth development where almost all cells that are in contact seem to be elongated/ stretched when comparing with native titanium sample. 
Therefore, it is seen from the observed results figure 5-(7,8) increase in rutile phase dominance nanostructure directly (manipulating oxide phase dominance) affects cell proliferation due to the underlying engineered core-shell like nanostructure material.

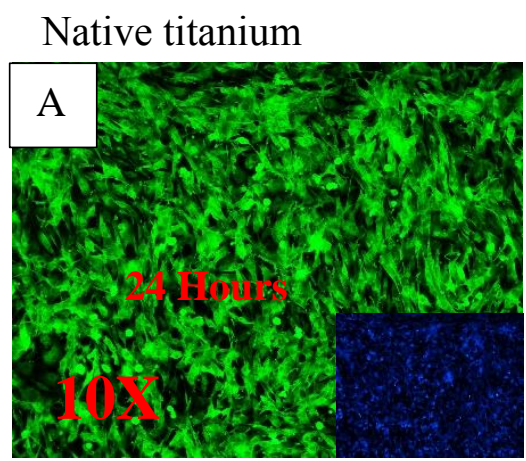

Rutile dominant nanostructure
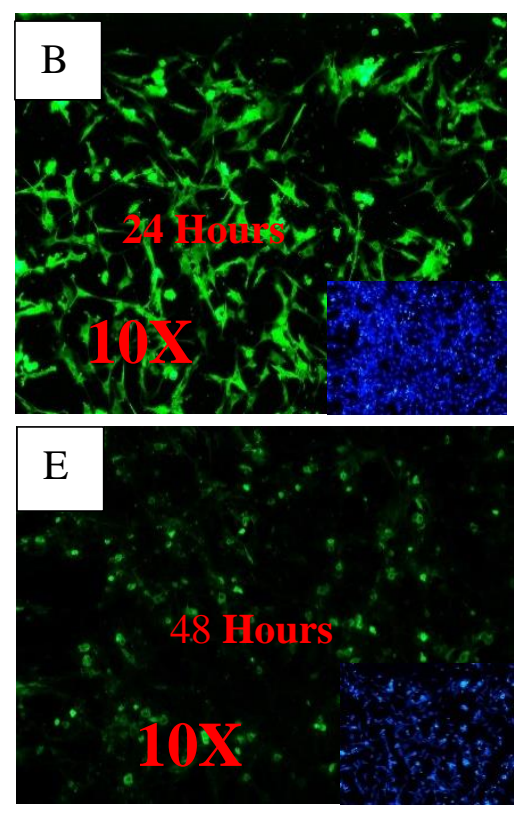

Anatase dominant nanostructure
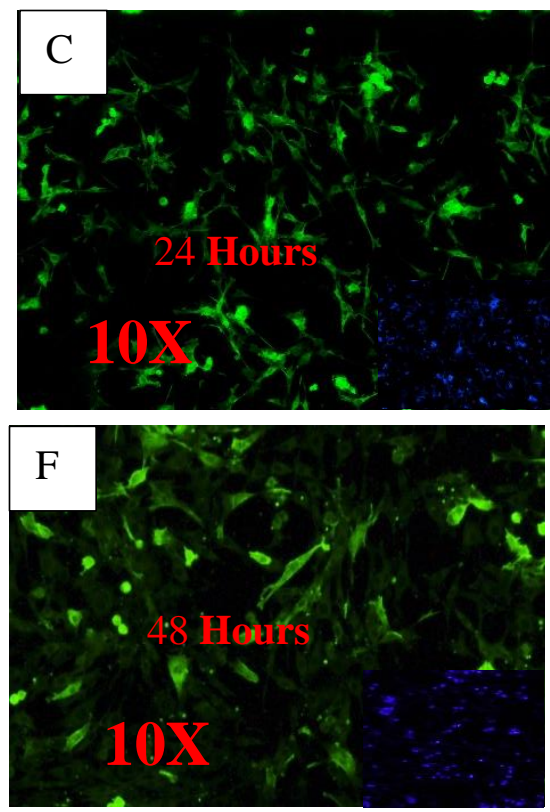

Figure 5-9: Fluorescence microscopy data of MC 3T3-E1 Osteoblast cell interaction on varying nanostructure densities with $24-48$ hour incubation time (B \& E) rutile phase dominant (C \& F) anatase phase dominant nanostructure (A-D) native control titanium

The above fluorescent microscope figure 5-9 clearly reveal the activity of cell through both cytoskeletal and nuclei presence. During the initial 24 hours, cytoskeletal activity decreases as rutile phase dominance increases. Presence of elongated cell cytoskeleton was also evident on anatase phase dominant nanostructure confirming with SEM analysis in figure 5-(7,8). As the incubation time is increased from 24 to 48 hours the presence of elongated cell cytoskeleton also start to fade with no evidence of change in the nucleus activity. But, when comparing the cellular activity on rutile phase dominant nanostructure a decreased cell activity is seen from 24 to 48 hours suggesting the structure repels cell activity. Although X-ray Diffraction analysis of synthesized phases show the relationship between laser peak power and dominance of individual phases in the synthesized oxide mixture the need to track the growth of healthy proliferating cells from repelled cells (about to die) needs to analysed through single cell analysis. 


\subsection{Change in cell morphology to engineered nano structure}
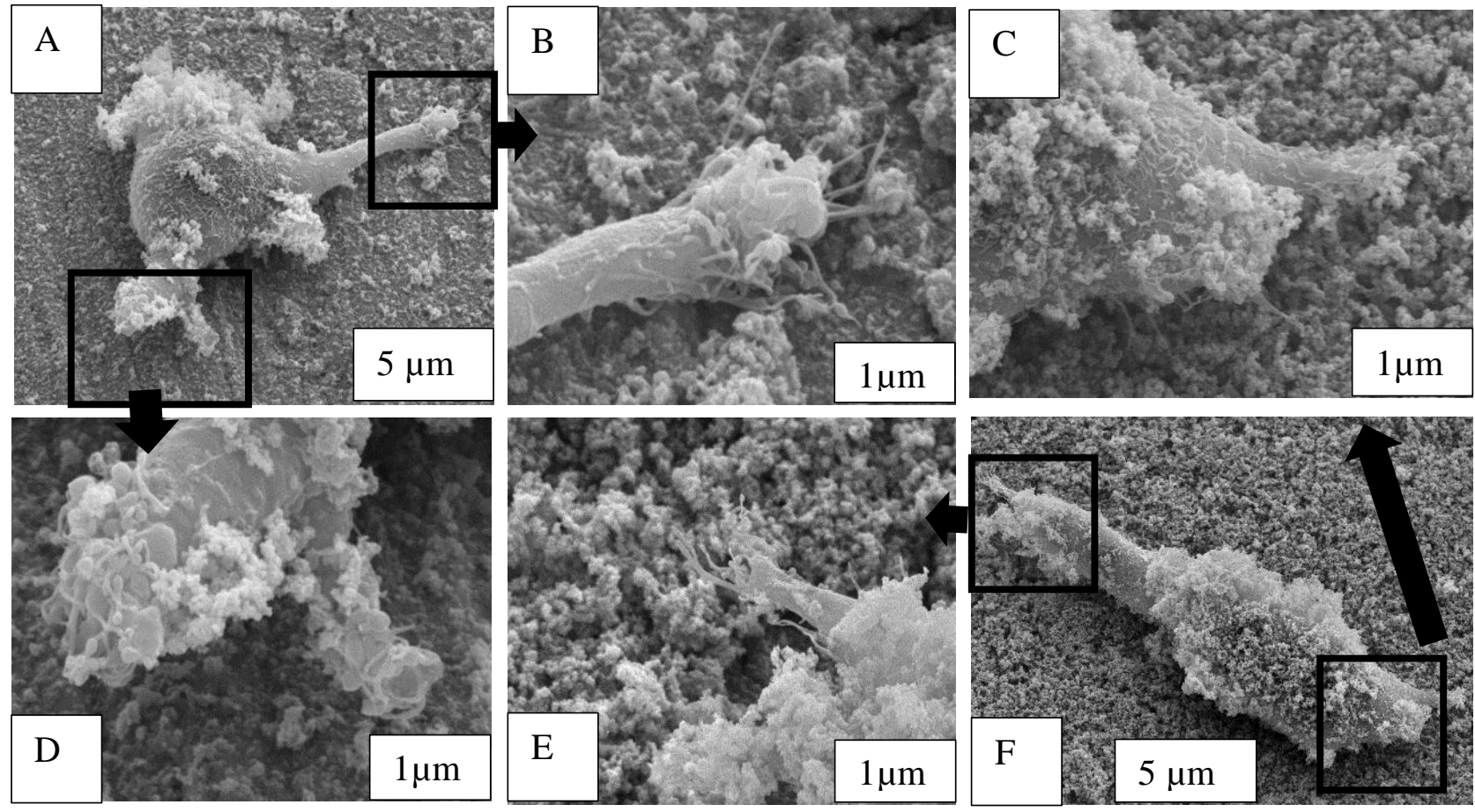

Figure 5-10: Change in MC3T3-E1 cell morphology due to both anatase and rutile dominant nanostructure after 24 hours (A, B and D) - anatase dominant (C, E \& F) - rutile dominant nanostructure

Cell migration is considered as a highly complex behaviour with significance in many physiological conditions. Cell migration is determined by the serious of migration cues presented to it in the interacting conditions. [68] These cues trigger the signal pathways that governs the actomyosin cytoskeleton and if the cues are spatially homogeneous, cell retain a random migration pattern. But, if the cues become spatially nonhomogeneous, a more directed migrations can be achieved. [68] Cells moves either individually or collectively on a surface and the need to understand this migration pattern is imperative from different biological contexts. [69] For instance, individual cell migration drives cell trafficking which in turn drive immune function, [70] and collective cell migration oversees growth and organogenesis [71]. The above synthesized structures figure 5-10 having either anatase of rutile phase dominance show the interacting nanostructure on osteoblast cells after 24 hour incubation.

The significance of synthesized structure density on cell interaction was already identified from figure 5-(7,8). The interaction of anatase phase dominant structure is presented in figure in $5.10 \mathrm{~A}$ 
where both extending/ proliferating filopodia are arrested by the underlying synthesized anatase phase dominant nanostrucutre. The development of filopodia was completely stopped as seen in Figure 5.10 (D) by the synthesized core shell like nanostrucutre by encapsulating the probing end. At the same time, the filopodia did manage to proliferate on the other side from Figure 5.10 B. Interaction of rutile dominant nanostrucutre on a single cell is seen in figure $5.10 \mathrm{~F}$ where the underlying synthesized nanoparticles in the nanostrucutres encapsualted a part of probing cell and stopped from proliferation. The isoalted image of a extending filopodia is seen is in figure 5.10 E.

\subsubsection{Microvilli manipulation in cells by phase dominant nanostructure}

Although quantitative measurements suggest microvilli are invariably associated to partial rounding and spread of cells. In general, rounding of cells in a normal population of cells suggest either cell division or cell death [72]. Cells which are in mitosis stage are regularly seen with high density of microvilli and which are in apoptosis will generally lack it.

Microvilli are normally formed with $1 / 3^{\text {rd }}$ of the total cell population and cells normally look rounded up when formed. The presence of microvilli also represents an establishment of denser nuclear region and presence of retraction fibers. A denser nucleus also indicates development stage
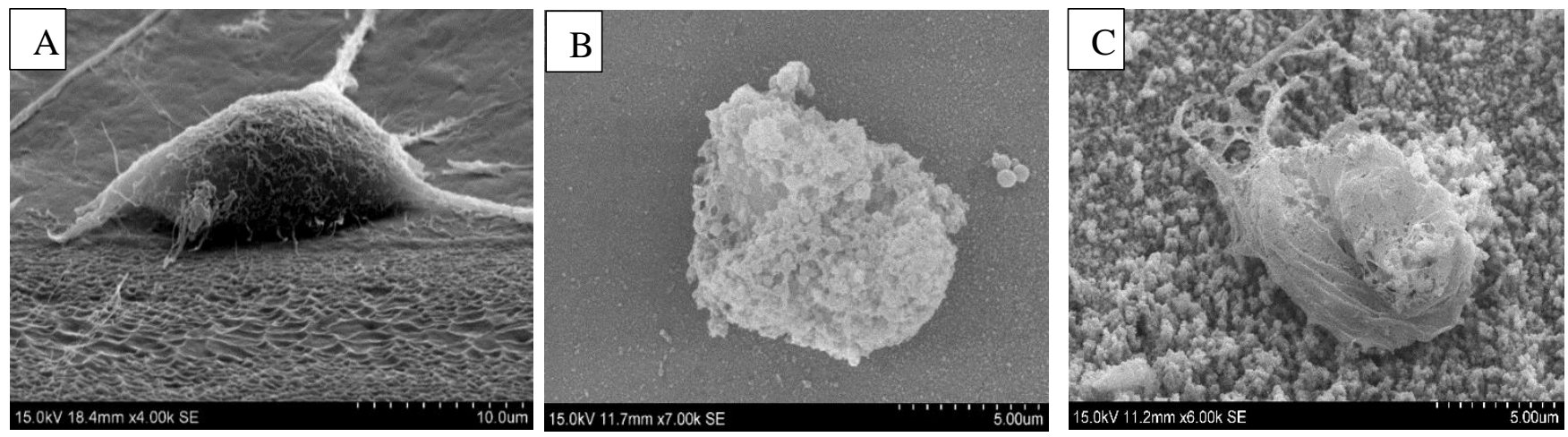

Figure 5-11: Microvilli Development in cells and effect of both anatase and rutile phase dominant structure in microvilli generation (A) - normal microvilli development (B) anatase phase dominant $(C)$ - rutile phase dominant structures

and the cells are considered with less microvilli extensions are in apoptosis state. The rounded morphology exhibited by the above cells figure 5-11 show two contrast characteristics where microvilli on rutile dominant nanostructure exhibit apoptosis and anatase dominant nanostructure 
showing signs of growth. Hence, the surface chemistry of synthesized nanostructure by own is inducing cell apoptosis.

\subsubsection{Influence of cell nucleus phase dominant structure:}
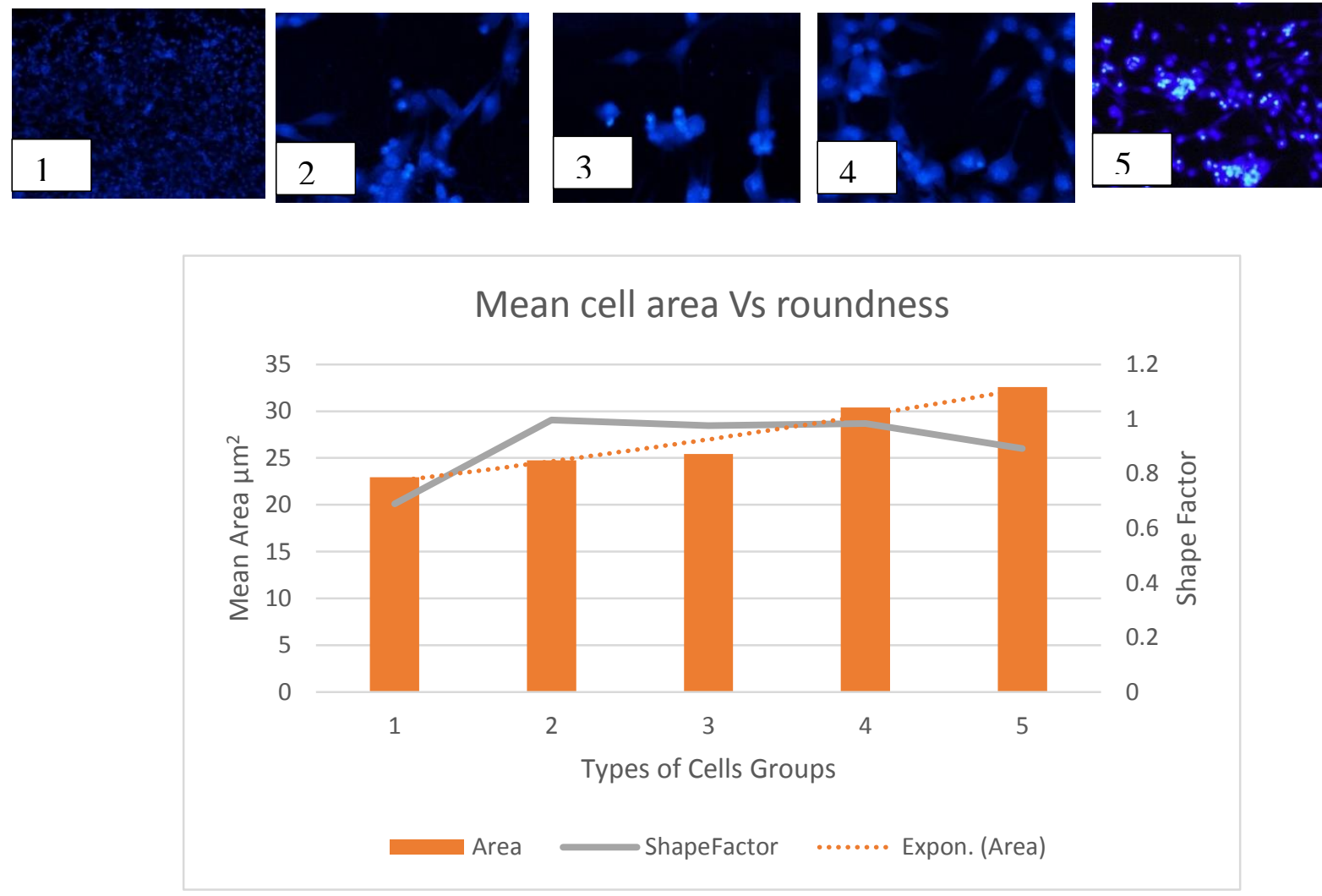

Figure 5-12: Reduction in cell adhesion with synthesized dominant phase

In an event to understand the activity of cell apoptosis and proliferation the above cells were split into 5 categories based on mean area of spread. A perfectly round shape cell is calculated to have a shape factor of 1 . Hence, a non-proliferating cell will have shape factors in decreasing magnitude. On Both rutile (2,3)and anatase (4,5) dominant nanostructures the cell nuclei shape was calculated and graphed above in figure 5-12. As incubation on rutile dominance phases increases from 24 to $48 \mathrm{hrs}$ shape factor of cell also increases which thereby indicates that the rutile dominant phase reduces cell proliferation and induces apoptosis. 


\subsubsection{Influencing cell motility by synthesized nanostructure}
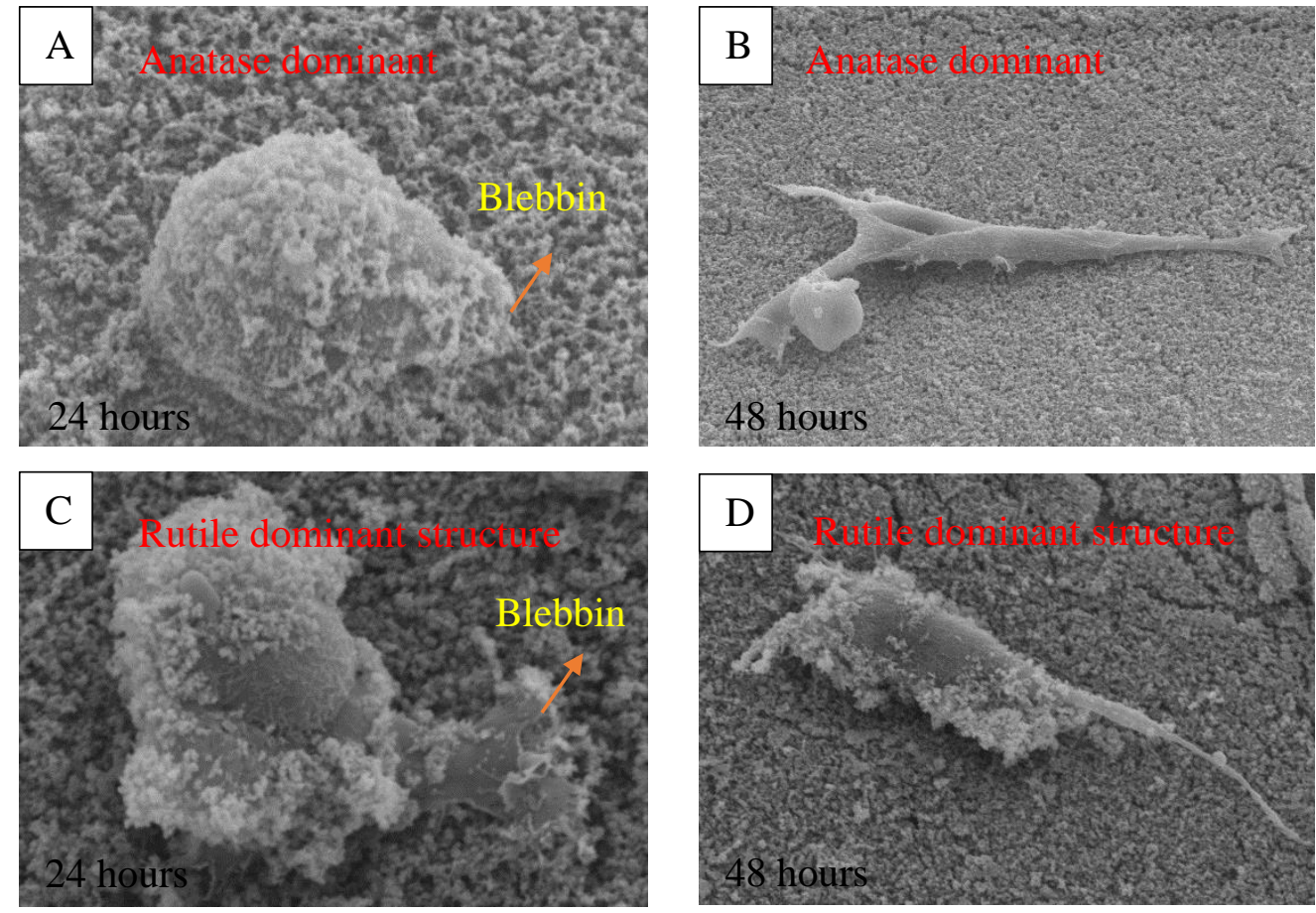

Figure 5-13 : Distinct change in cell morphology observed in osteoblast cells on synthesized struture from 24 to 48 hours

Two distinct cell shapes were observed on synthesized anatase and rutile dominance nanostructure. From the above figure 5-13 (A) a distinct round cell is found to have extension on oneside a phenomenon called as blebbing [71] which then later develops to an elongated spindle like shape cell figure 5-13 (B). Blebbing is reported as a common phenomenon where spherical cells develop into proliferating cell. From the above cell-material interaction figure 5-13 blebbing is found to be persistant with both anatase and rutile dominant nanostructutres during the intial 24 hours. But, when the incubation time is increased further to 48 hours an acute change in cell morphology was oberserved from anatase to rutile dominant nanostructure. The anatase dominant nanostructures had normal proliferation with uniform filopodia to lamellopdia development from figure 5-13 (C) but, in rutile dominant nanostrucutre the lamellopodia extensions were roofed by nanostructure with long filopodia extensions suggesting a complete change in cell spreading activity by the synthesized phase dominant nanostrucutre ar figure 5-13 (D). 


\subsubsection{Cell trapping by phase dominant nanostruture on fibroblast cells}
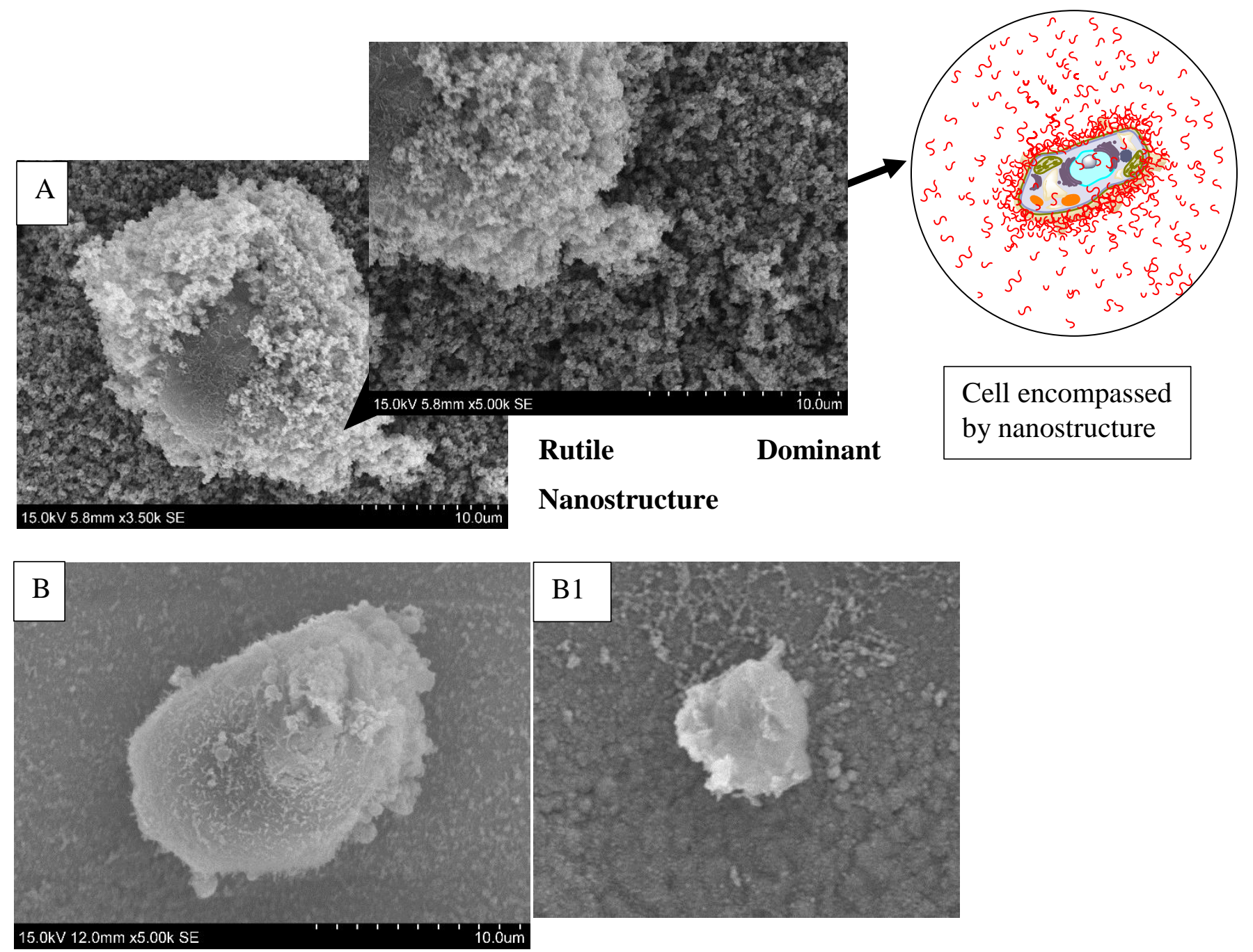

Anatase Dominant Nanostructure

Figure 5-14: Influence of Synthesized nanostructure on NIH 3T3 Fibroblast Cell trapping at rutile (A) and anatase (B) phase dominant nanostructure

The above figure 5-14 portray cell encapsulation by certain phase dominant nanostructure. Although the concept of cell encapsulation is to arrest cell proliferation in osteoblast cells the same notion can also be extended even for fibroblasts cells. The influence of nanoparticle size on cell controllability was already devised through isolation of titanium dioxide nanoparticles having uniform size $\sim 50 \mathrm{~nm}[60]$. But, the concept of inducing apoptotic behaviour on interacting cells through the synthesized nano-core shell like structure having individual phase dominance was never reported before. 


\subsubsection{Fibroblast cell proliferation on nanostructure density}
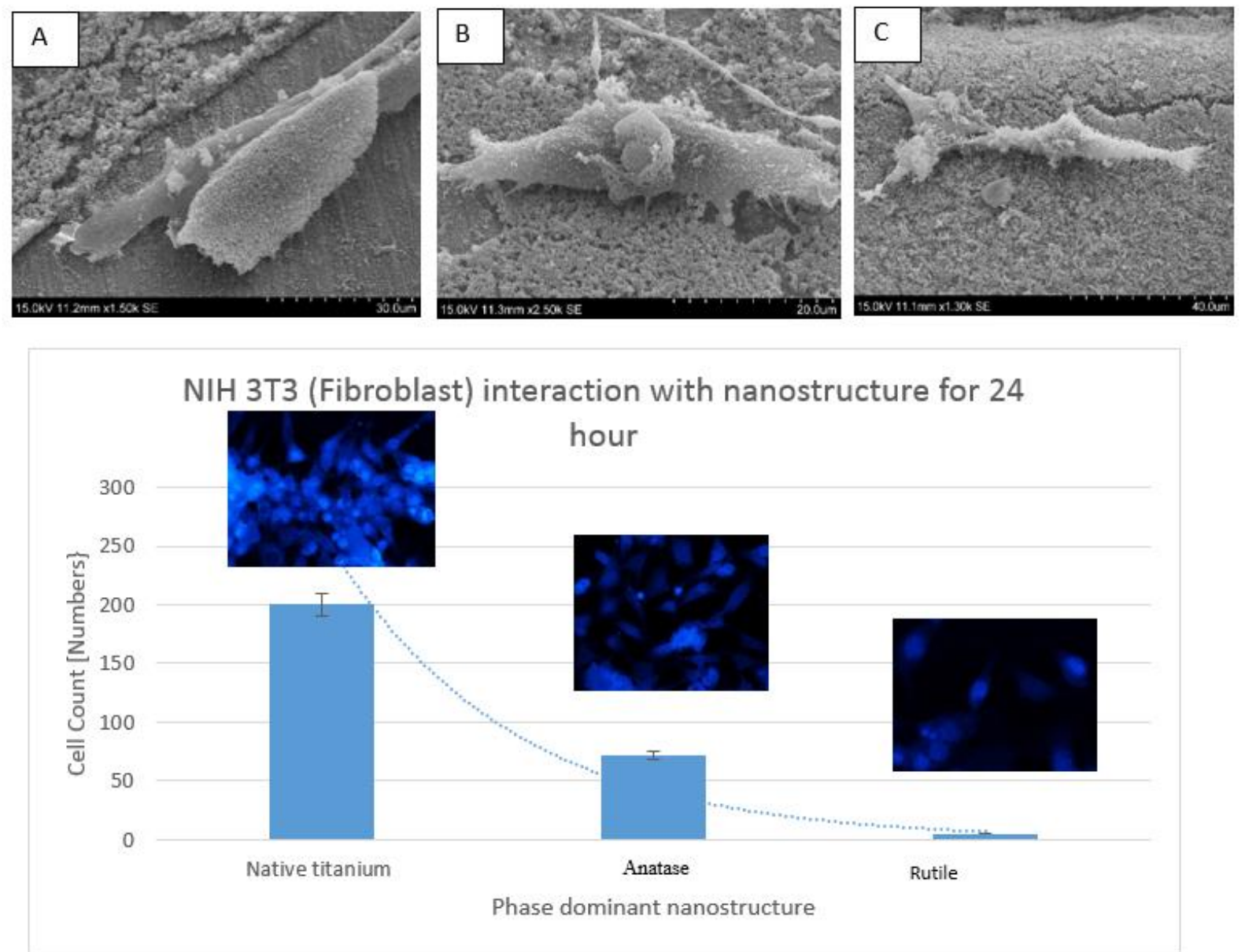

Figure 5-15: SEM Images of NIH 3T3 Fibroblast cell interaction on native and with nanostructure density having phase dominance (B-C) after 24 hours

The above figure 5-15 illustrates the effect of phase dominant nanostructure on fibroblast cells. Cells proliferate well on native titanium surface figure 5-15 (A) but it comes in contact with anatase dominant nanostructure it acutely changes its morphology pattern with fewer filopodial sensing happening on the interacting nanostructure. When the same fibroblast cell when in contact with rutile dominant nanostructure the nanoparticles encapsulate them restricting their proliferation. The above graph in figure 5-15 illustrate the same where rutile dominant nanostructures had far too less nuclei when comparing with native titanium. 

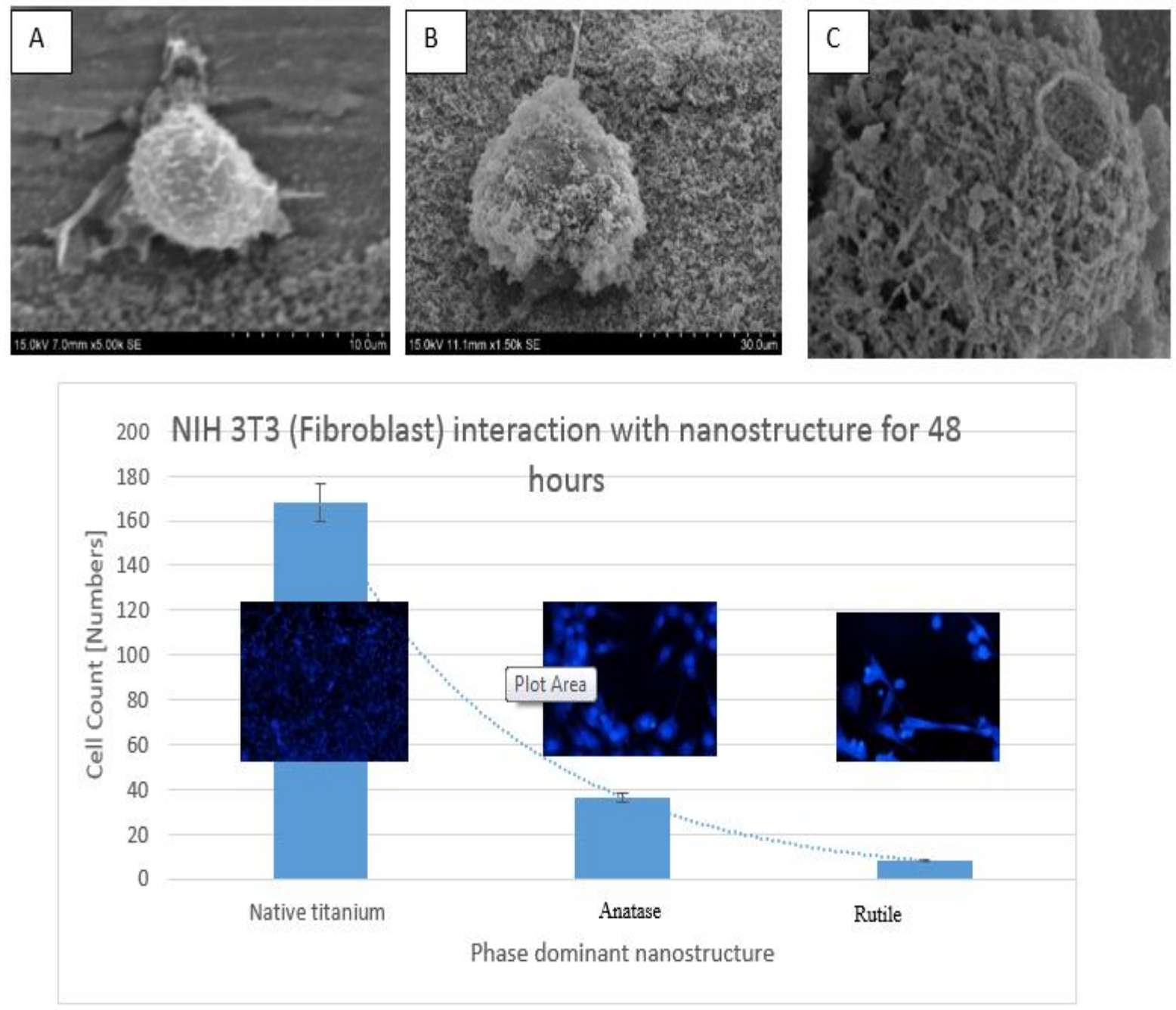

Figure 5-16: SEM Images of NIH 3T3 Fibroblast cell interaction on native and with nanostructure density having phase dominance (B-C) after $\mathbf{4 8}$ hours

When the incubation time was doubled from 24 to 48 hours the phase dominant structure present in the underlying synthesized nanostructure react with cell and encapsulate the entire cell thereby arresting growth. The nucleus of the cell therefore becomes more round unable to proliferate and floats over the synthesized structure. The nuclei intensity from above figure 5.16 confirms the same with rutile dominant nanostructure region having least nuclei proliferation. 


\subsubsection{Filopodial sensing by fibroblast cell on synthesed strucutres}
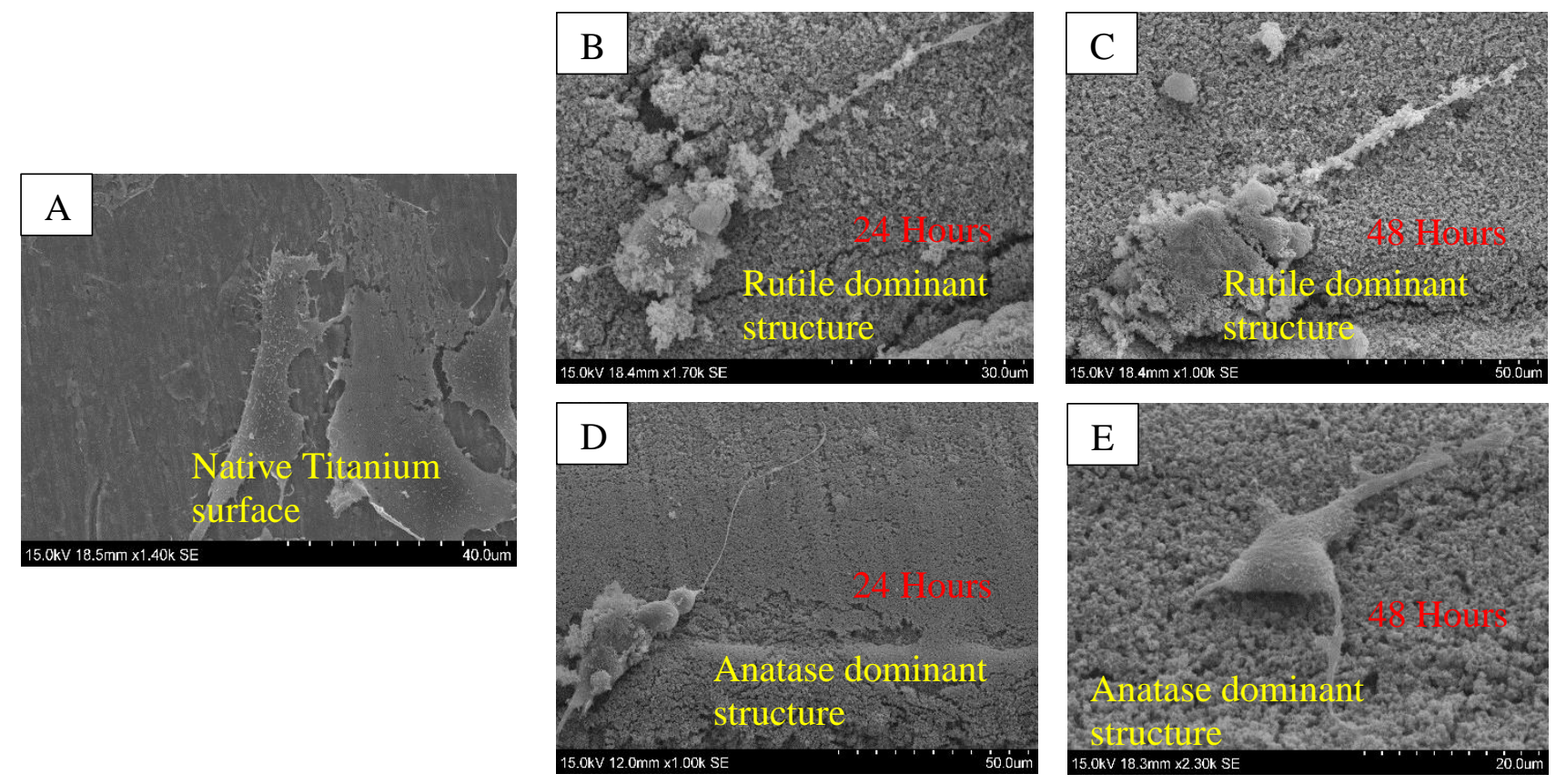

Figure 5-17 Development of filopodia on both anatase and rutile phase dominant nanostrucutre at 24 and 48 hours

Fibroblast cells have been widely used used to explore the influence of nanoscale topography on cell response[72]. It is reported that nano rough surfaces created with silica nanoparticles influence fibroblast cell response affecting morphology, reducing adhesion and thus inhibits cell spreading [72]. The above observed fibroblast cells show normal cell proliferation with short filopodial developments on the native control sample figure 5.17 (A) but, show a complete new development on phase dominant nanostrucutres with long thin filament like filopodias. In general filopodia help the cell in navigation, proliferation and altering its development can have a huge impact in terms of cell adhesion and proliferation. When comparing the filopodial development with anatase and rutile dominent nanostrucutre for 24 hours figure 5-17 (B, D) a drastic change in filopodia length and thickness is observed. The anatase dominent nanostrucutres doesn't seem to distress filopodial growth but rutile dominant nanostucture appear encapsulating the filopodia thereby altering their morphology and sensing. When the incubation time was doubled from 24 to 48 hours, a similar trend with anatase dominant nanostucture figure 5.17 (E) where evidence of lamellepodial development is witnessed with short interacting filopodia suggesting proliferating cell. But, in rutile dominent nanostrucutre figure 5-17 (C) evidence of filopodia encapsualtion by nanostructure 
becomes more prominent when comparing with figure 5-17 (B) suggesting reduced cell adhesion capacity due to chemistry of synthesized rutile phase dominent nanostructure.

\subsection{Summary}

This approach proposes a new application for 3D ECM like nanostructures for smart interactive regions where both the need to control and induce proliferation is addressed. The novel USP laser synthesis employed generates titanium oxide core-shell like nanostructures over native titanium substrate using vapor condensation mechanism. The process of selectively biofunctionalizing a phase to be dominant on the synthesized strucuture was never reported before. A unique mechanism of cell encapsulation to induce cell apoptosis is introduced. A 96\% cell reduction with 19 times cell deterioration from native tiatnium substrate is reported on the rutile phase dominant structures. These nanostrucutures hold potential to smart interactive titanium based biomaterial inducing cell proliferation and apoptosis based on oxide phase dominance. 


\section{Chapter 6- Phase transformed surface and nano printed structure for cell manipulation}

\subsection{Introduction}

A significant challenge in current implant technology now is in designing biomaterials that can actively promote (differentiate) align (migrate) and repel (apoptosis) cells on a selective area. Titanium is an important material for a range of biomedical applications including prostheses, orthopedic implants [73], pace makers and drug eluting stents.

Surface patterning combines a unique blend of both surface chemistry and material science to elucidate in vitro interactions of anchorage dependent cells. [74] In order to be viable, anchorage dependent cells by nature need an adhesive surface to exert force and proliferate over. But, the ability to constrain this spreading to a specific area of interest can dramatically alter cell development.[75,76]. Several studies show nanometer scale surface features can significantly alter cell adhesion, differentiation and alignment [77, 78]. Cellular reactions to surface features in micrometer scale surface topographic features such as grooves, ridges and wells has been well established [78,79]. Over the past few years, sudden interest in evaluating potential nanostructured material for biomedical application has shifted focus on manipulating cell activity in 3D ECM materials [9].

The present study using USP laser offer a more robust method for synthesizing selective surface patterning on any substrate. Compared to conventional methods like lithography which is limited to choice to substrate, stamping material, and limited shape sequencing [74], a simple one - pot synthesis method is presented. 

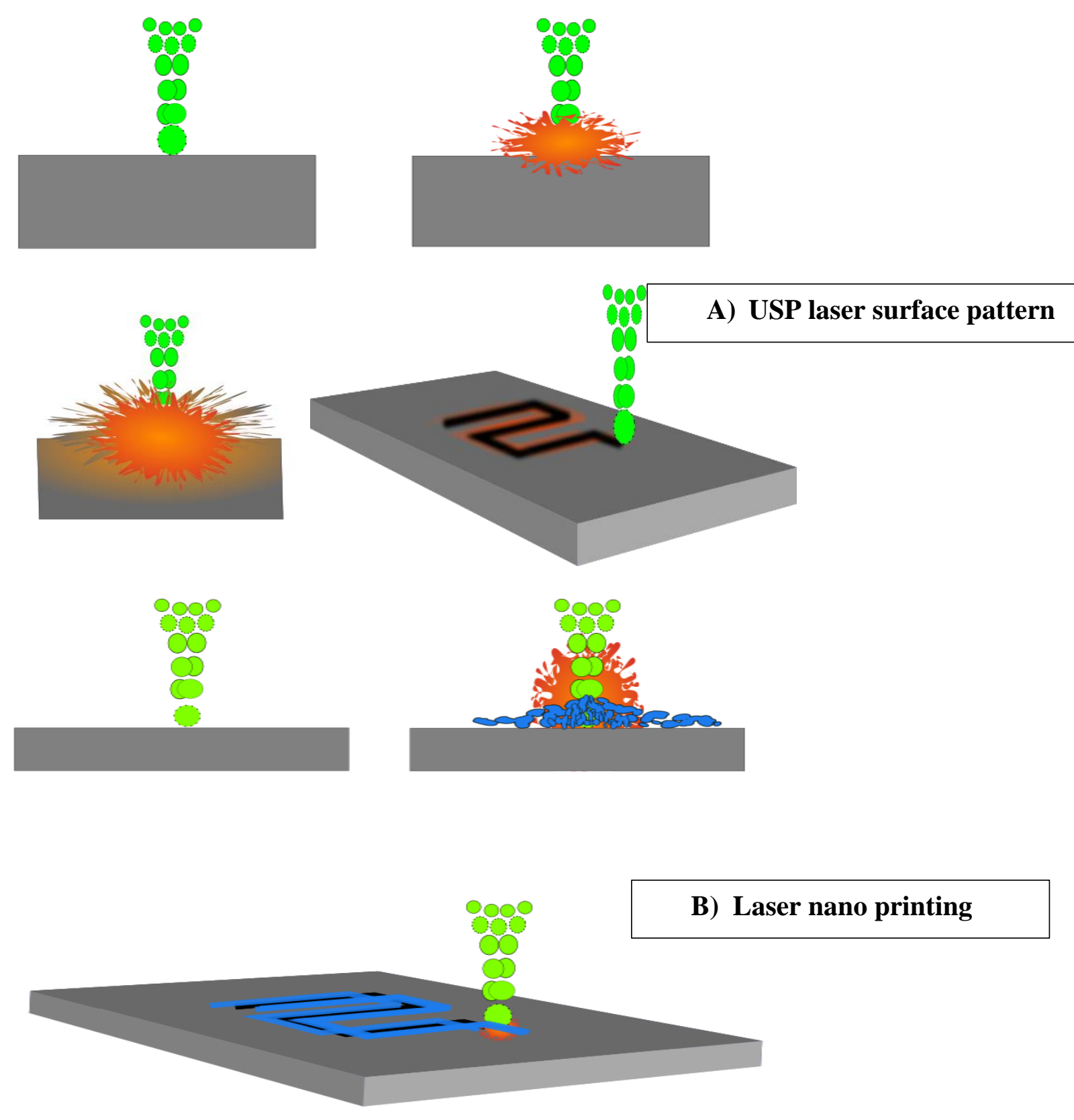

Figure 6.1 Schematic illustration of laser material interaction A) USP laser surface patterning and $B$ ) laser nano printing

The above schematics in figure 6.1 explains versatility of USP laser in both surface patterning and nano-printing where interacting laser pulses can effectively functionalize a selective area of material to desired application. 


\subsection{Novel Laser Surface Patterning}

Although, the concept of novel USP laser material phase transformation is already discussed in chapter 4 the same phenomenon can be extended towards surface patterns to analyse and evaluate cell material response on selective functionalization.
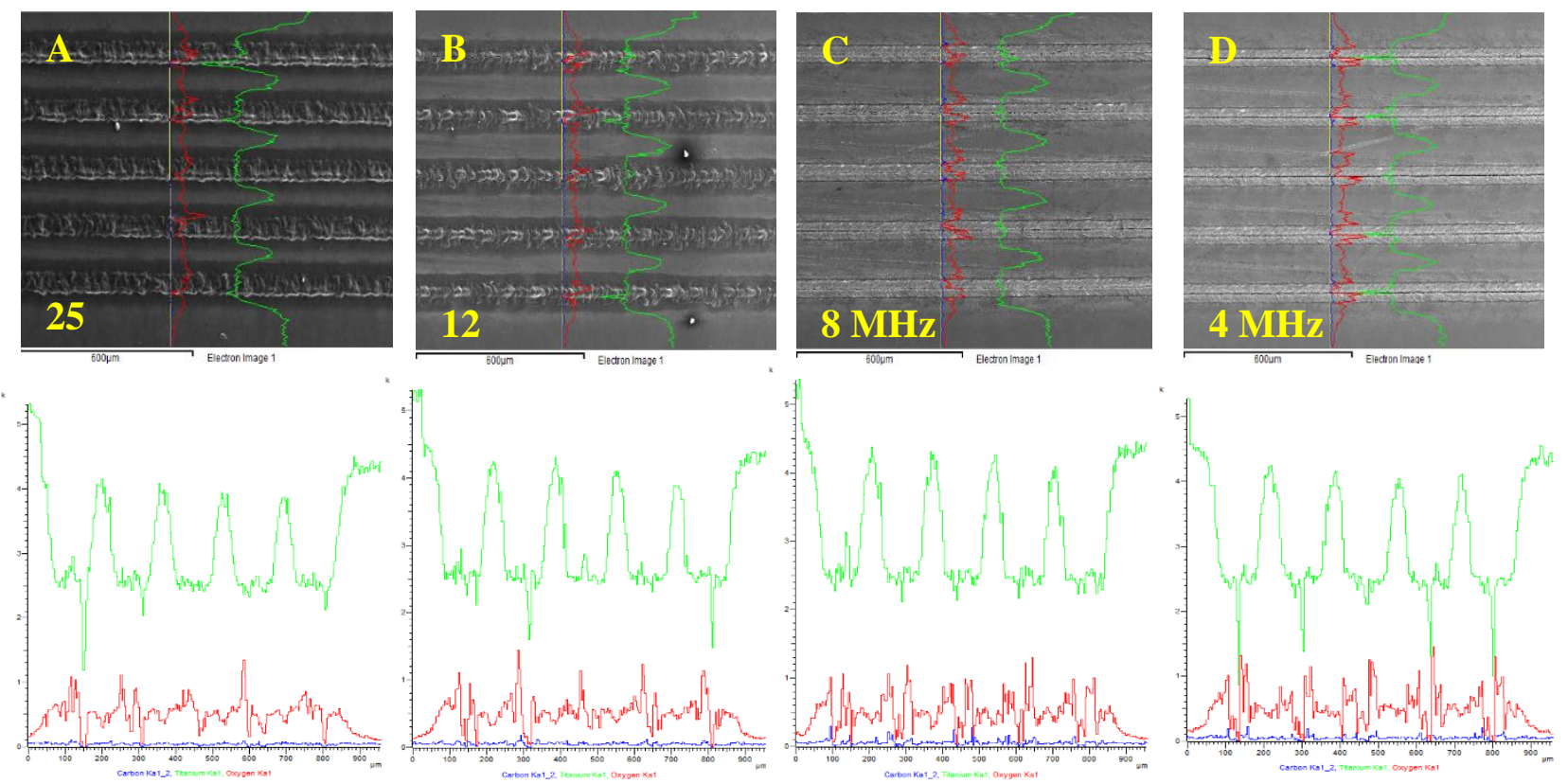

Figure 6.2 SEM/EDX line scan data of laser phase transformed surface pattern from $25 \mathrm{MHz}$ (high laser pulse frequency) to $4 \mathrm{MHz}$ (Low laser pulse frequency) holding a steady $1428 \mathrm{fs}$ laser pulse irradiation width at a constant pulse per unit of $5 \mathrm{~mm} / \mathrm{s}$.

The above figure 6-2(A-D) shows material phase transformation intensity by USP laser with respect to both topographic and intensity variation from the irradiated surface pattern. The EDX line scan images also reveal the change in titanium to oxygen ratio at each laser irradiated path where sharp variations are observed around the material phase transformation area. The material transformed surface at figure 6-2 (A-D) show trend in recast melt when comparing from one laser pulse frequency to another indicating that the effect of material transformation intensity by each laser pulse is more in figure 6-2(D) when comparing with figure 6-2 (A). The below trend line graph in figure 6-3 explains effect the of synthesized surface pattern oxidation with titanium and oxygen weight percentage variation from the above EDX line scan intensities in figure 6-2. 


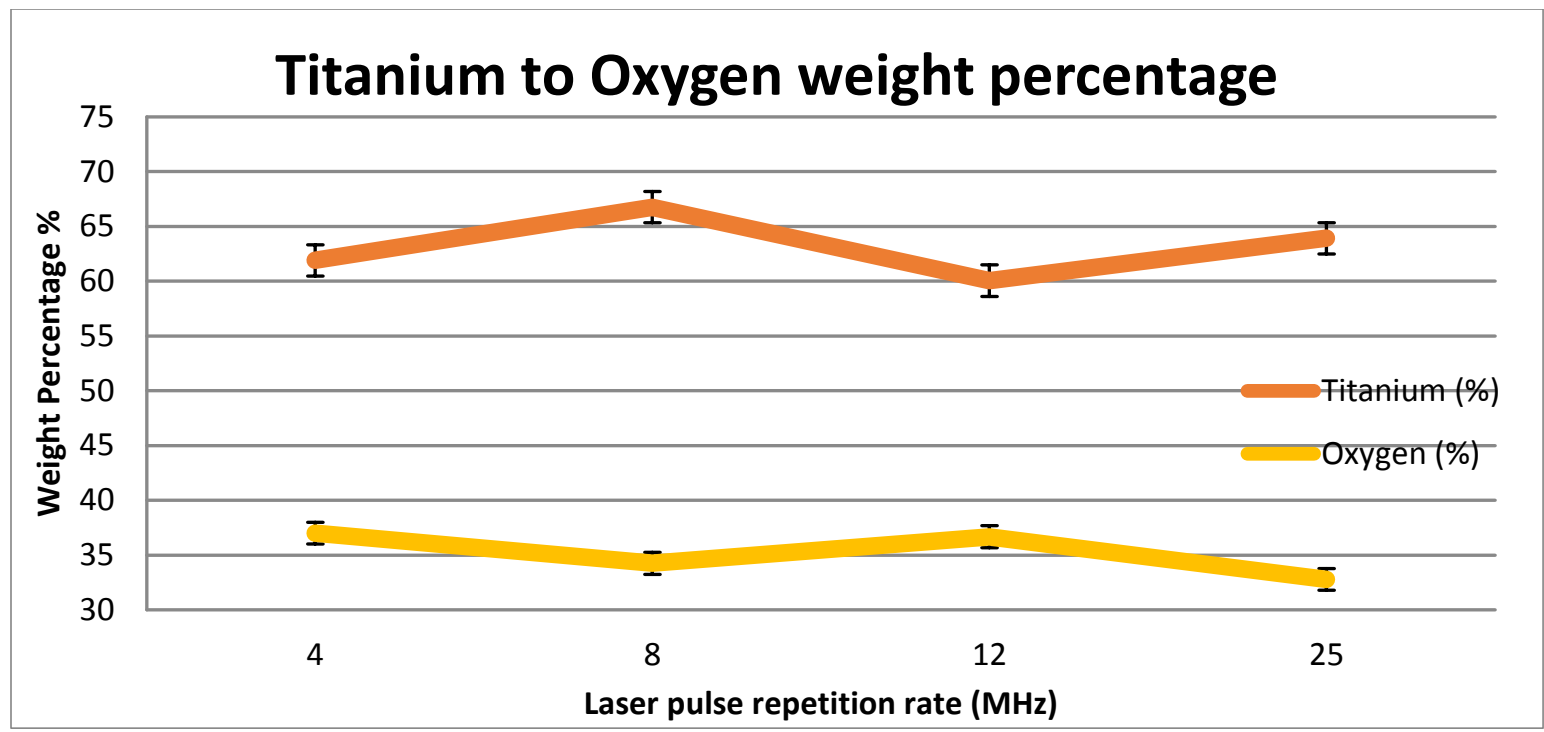

Figure 6-3: EDX line graph showing the variation of titanium to oxygen with laser pulse repetition rate

\subsection{Nano-printing using USP laser synthesis}

Unlike other conventional nano-patterning process like E-beam lithography and photolithography in USP laser a single step is utilized for creating nanostructures having specific density and oxide phase concentration. The process involved in synthesizing/printing nanostructured layer over titanium is analysed in chapter 5 .

Oxygen
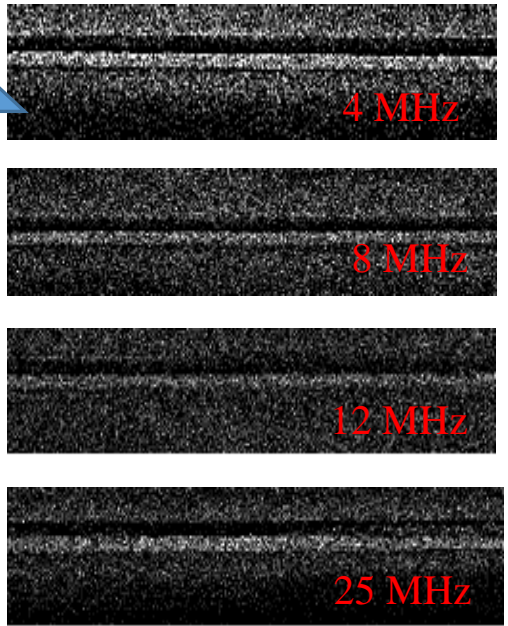

Titanium
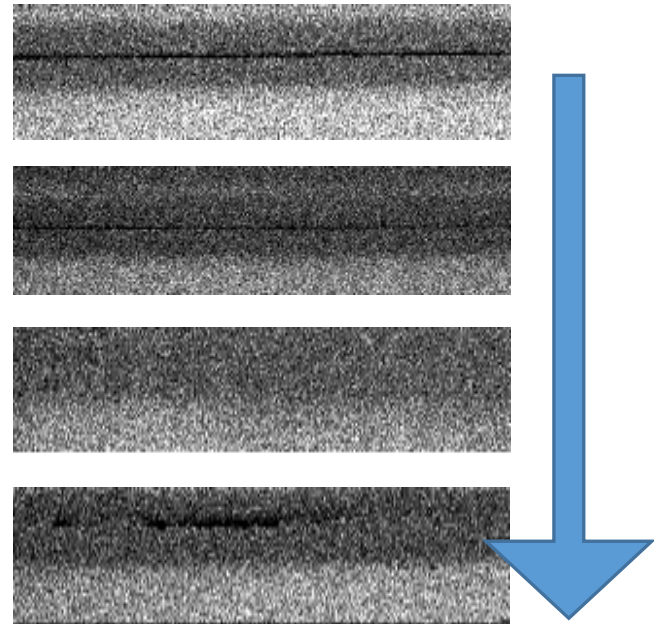

Figure 6.4 SEM/EDX area scan data of laser phase transformed on nano-printed pattern from $25 \mathrm{MHz}$ (high laser pulse frequency) to $4 \mathrm{MHz}$ (Low laser pulse frequency) at a steady 214 fs laser pulse irradiation width having a constant pulse per unit of $5 \mathrm{~mm} / \mathrm{s}$. 
The above figure 6-4 shows material phase transformation intensity by USP laser with respect to oxidation intensity variation from the irradiated nano-printed pattern. The EDX area scan images also reveal the change in titanium to oxygen ratio at each laser irradiated path where variations are observed around the oxidised material phase transformation area. The material transformed area at figure 6-4 show trend in oxidation when comparing from one laser pulse frequency to another $(25 \mathrm{MHz}-4 \mathrm{MHz}$ ) indicating that the effect of material transformation intensity by each laser pulse is more at low laser pulse frequency (4 MHz) when comparing with high laser pulse frequency (25 MHz). The effect of synthesized nano-printed pattern with titanium and oxygen weight percentage variation from the above EDX area scan intensities in figure 6-4 is showcased in figure 6-4.

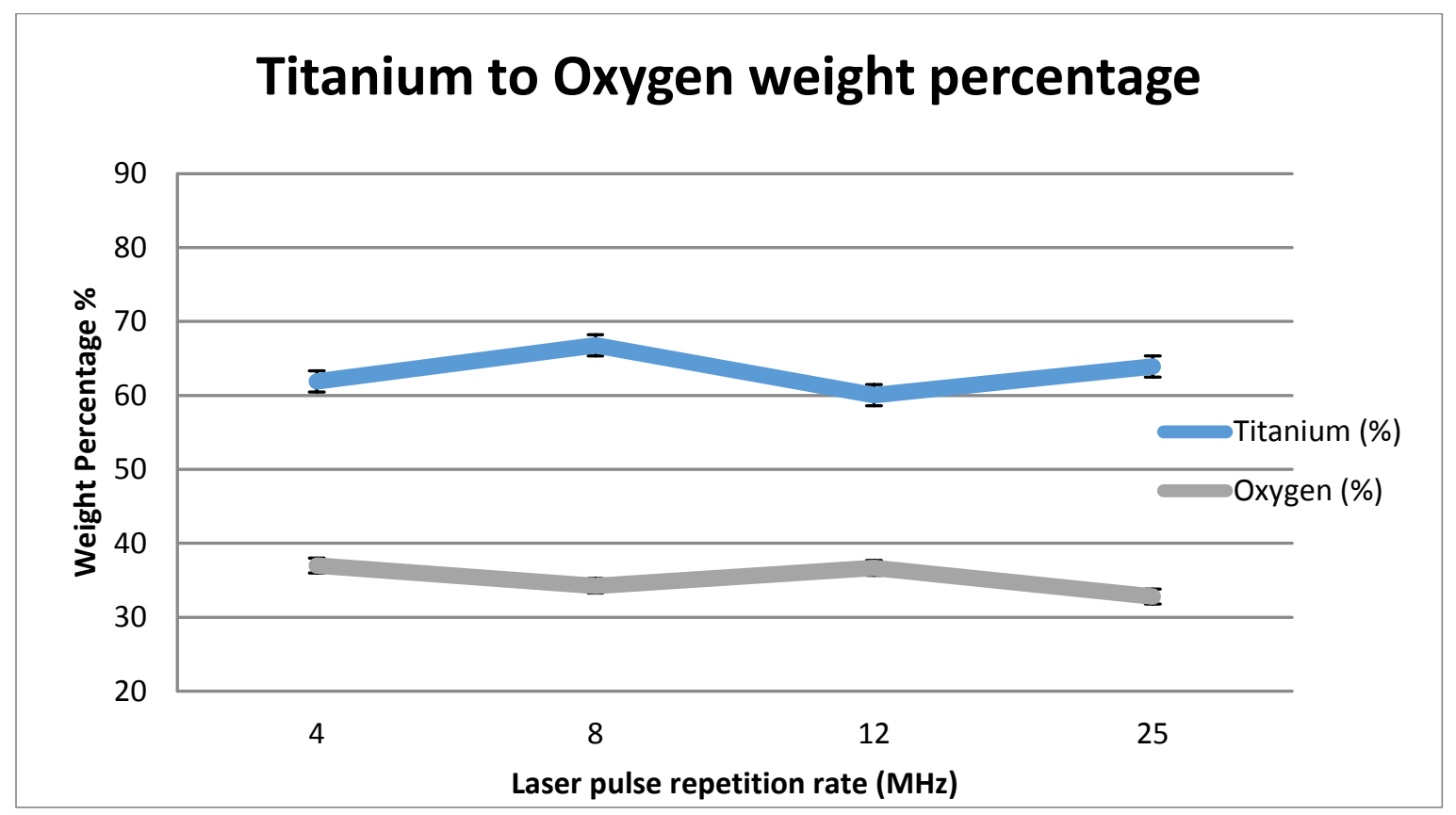

Figure 6-5: Area EDX graph showing the variation of titanium to oxygen with laser pulse repetition rate 


\subsection{Influence of synthesized patterns on osteoblast cell}

In an attempt to evaluate the influence of synthesized patterns with specific oxide phase concentration MC3T3-E1 osteoblast cells were made to interact for 24 hours.
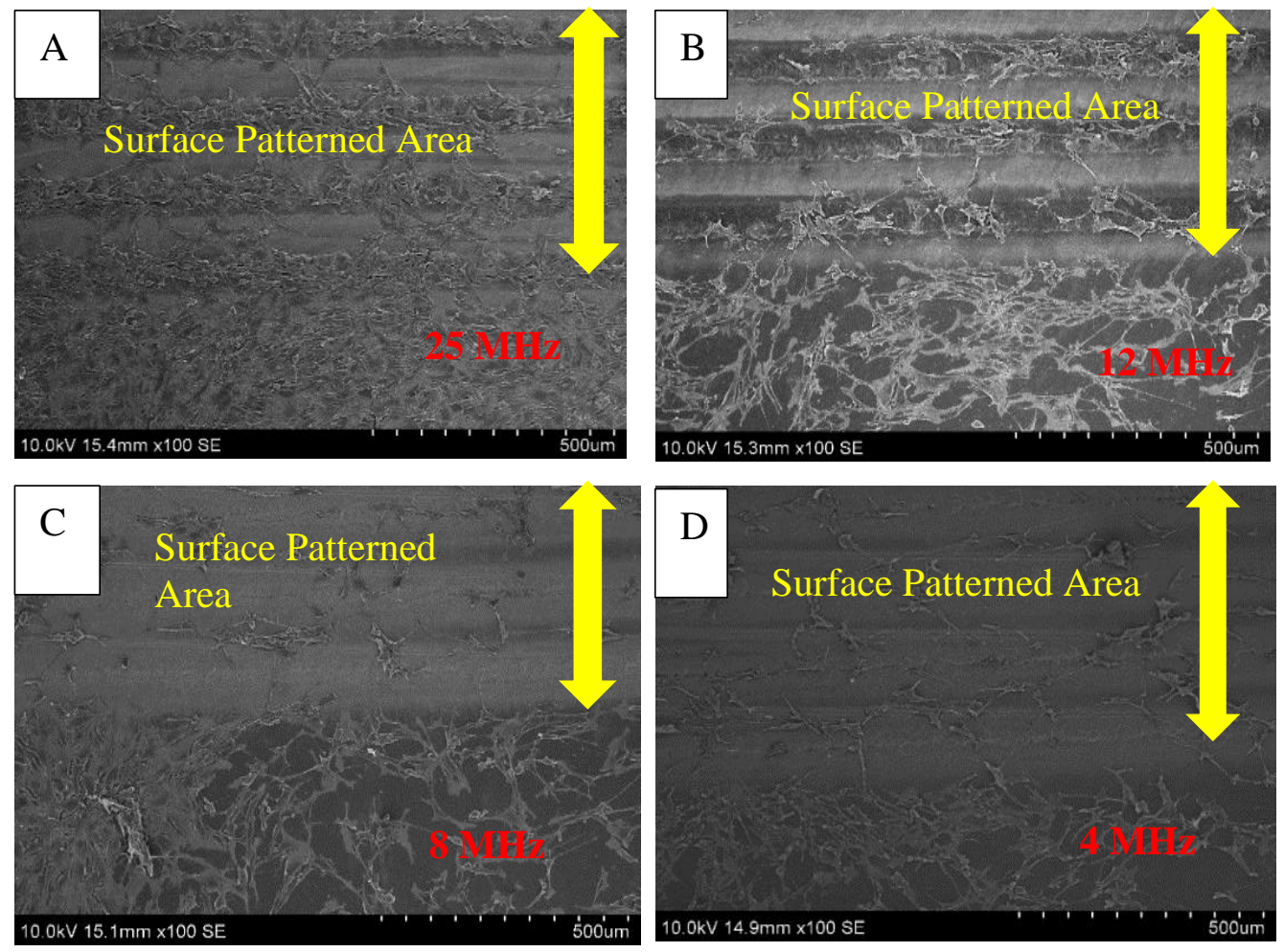

Figure 6-6: Effect of Osteoblast cell differentiation of each synthesized surface pattern after

\section{Hour incubation.}

From the above SEM micrographs figure 6-6 a clear trend is first evident where a significant reduction in cell growth as we move figure 6-6 (A-D). The cells look aligned and seem to favour native control titanium surface rather than the laser surface pattern area. The oxide phase composition of the synthesised pattern was already discussed in material characterization section in chapter 4 . 

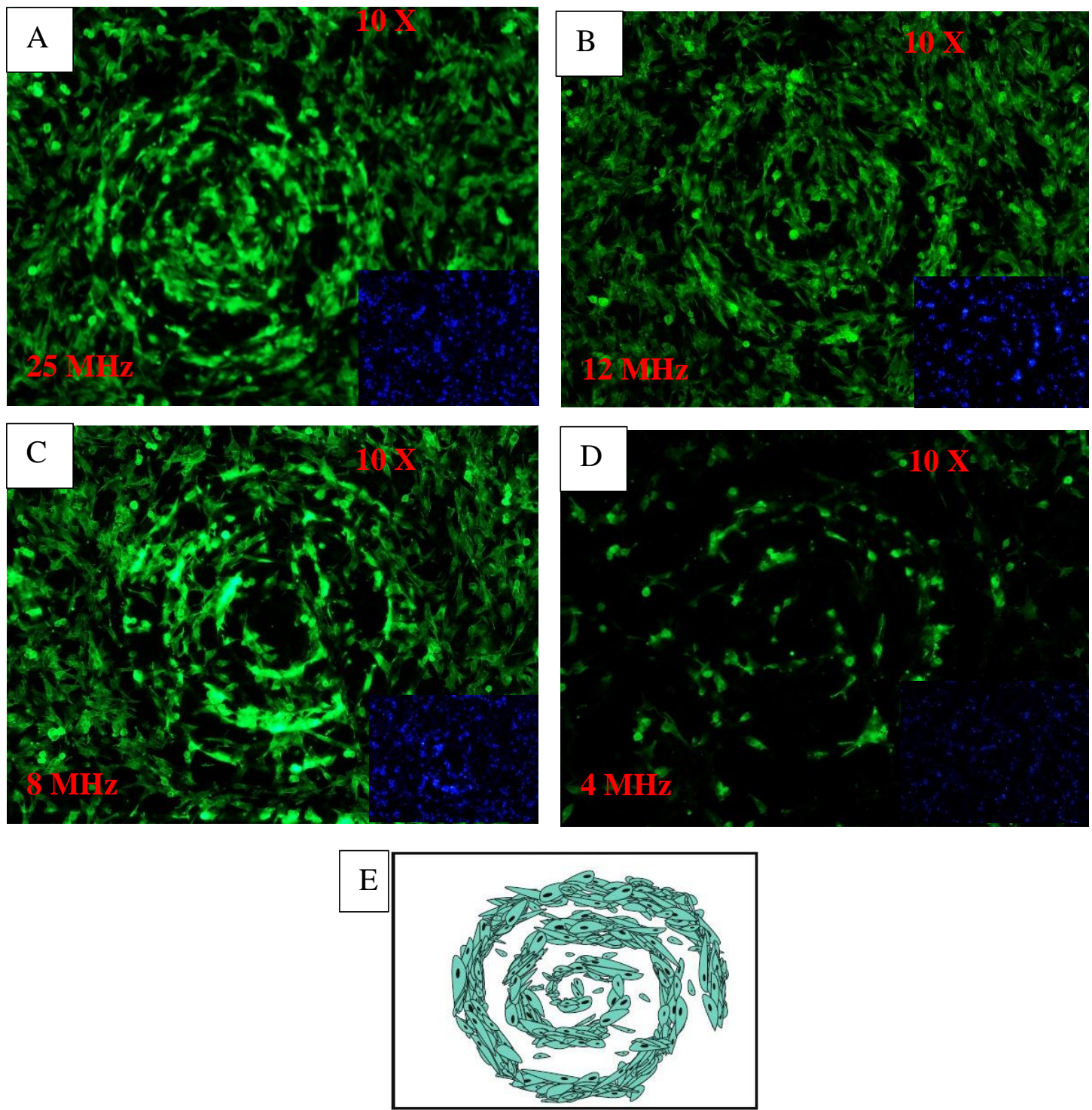

Figure 6-7: Fluorescent microscopic image on effect of MC3T3-E1 osteoblast cell proliferation on each synthesized pattern (A-D) after 24 Hour incubation. (E)- Illustration of pattern synthesized with preferential cell attachment only on laser irradiated surface In order to show the versatility of the process a circular pattern is drawn. From the above cell cytoskeleton and nuclear information figure 6-7 we could infer a trend pattern with gradual reduction in cytoskeletal activity from figure 6-7(A-D). A similar trend could also be inferred from cell nucleus intensity from figure 6-7(A-D). Surface patterned area show directional cell proliferation / migration on the laser material interacted region. The low proliferation activity at figure 6-7(D) is due rutile phase dominant surface which is explained in chapter 4. 


\subsection{Influence of selective nano printing on osteoblast cell}
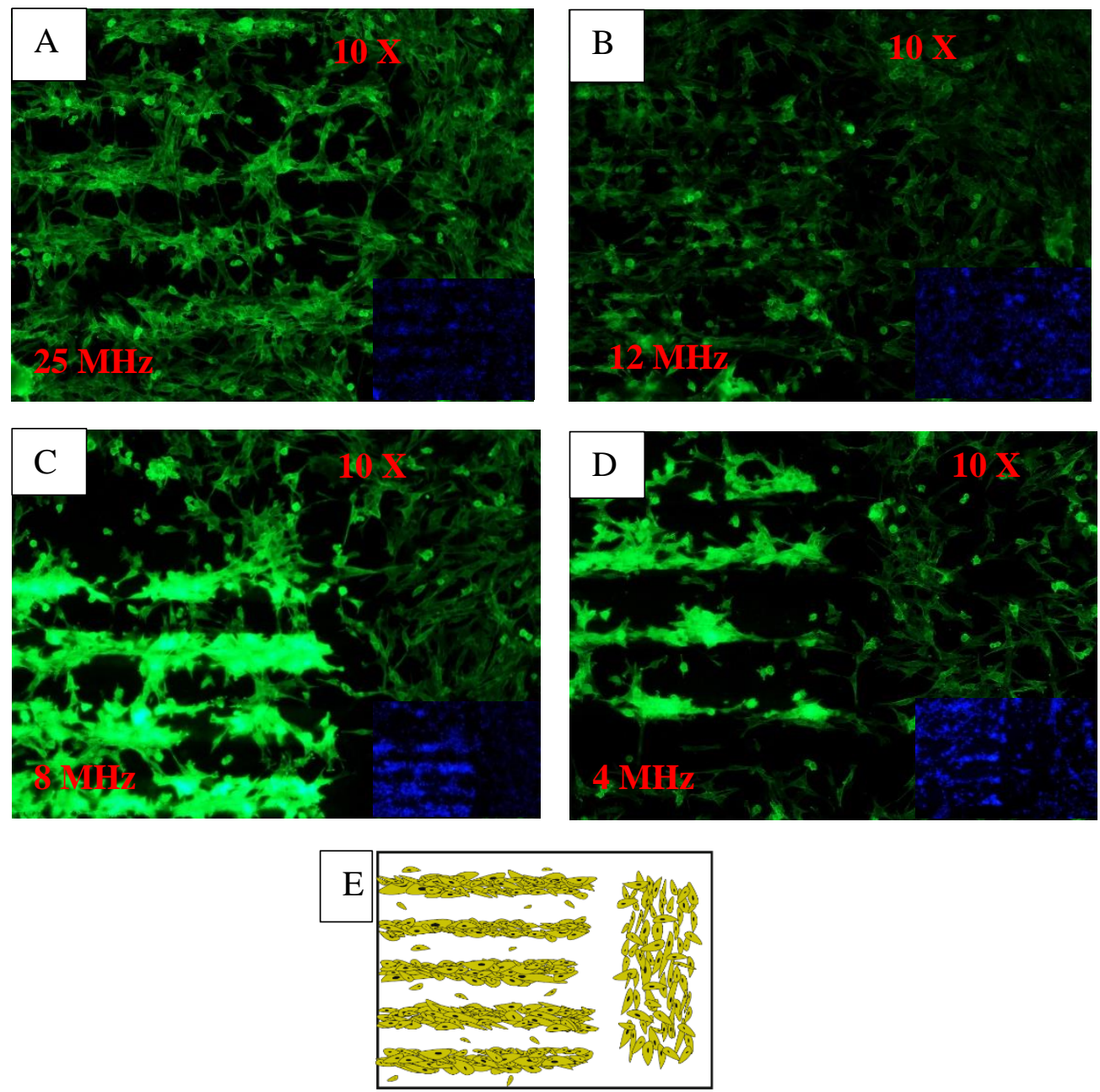

Figure 6-8: Fluorescent microscopic image on effect of MC3T3-E1 osteoblast cell proliferation on each nano printed pattern (A-D) after 24 Hour incubation. (E)- Illustration of nano printed pattern with preferential cell attachment only on laser irradiated surface In order to show the versatility of fabrication process the above illustrative pattern is drawn in Figure 6-78 (E) pattern is drawn. From cellular cytoskeleton and nuclear information in figure 68(A-D) we could infer a trend pattern with gradual reduction in cytoskeletal activity .A similar trend could also be inferred from cell nucleus intensity from figure 6-8(A-D). Only nano printed area show directional cell migration and proliferation with cell repellence near the printed area where high density nanostructure interact with the cell. The low proliferation activity at figure 68(D) is due to underlying rutile phase dominant nanostructure synthesized which is explained in detail in chapter 5 . 

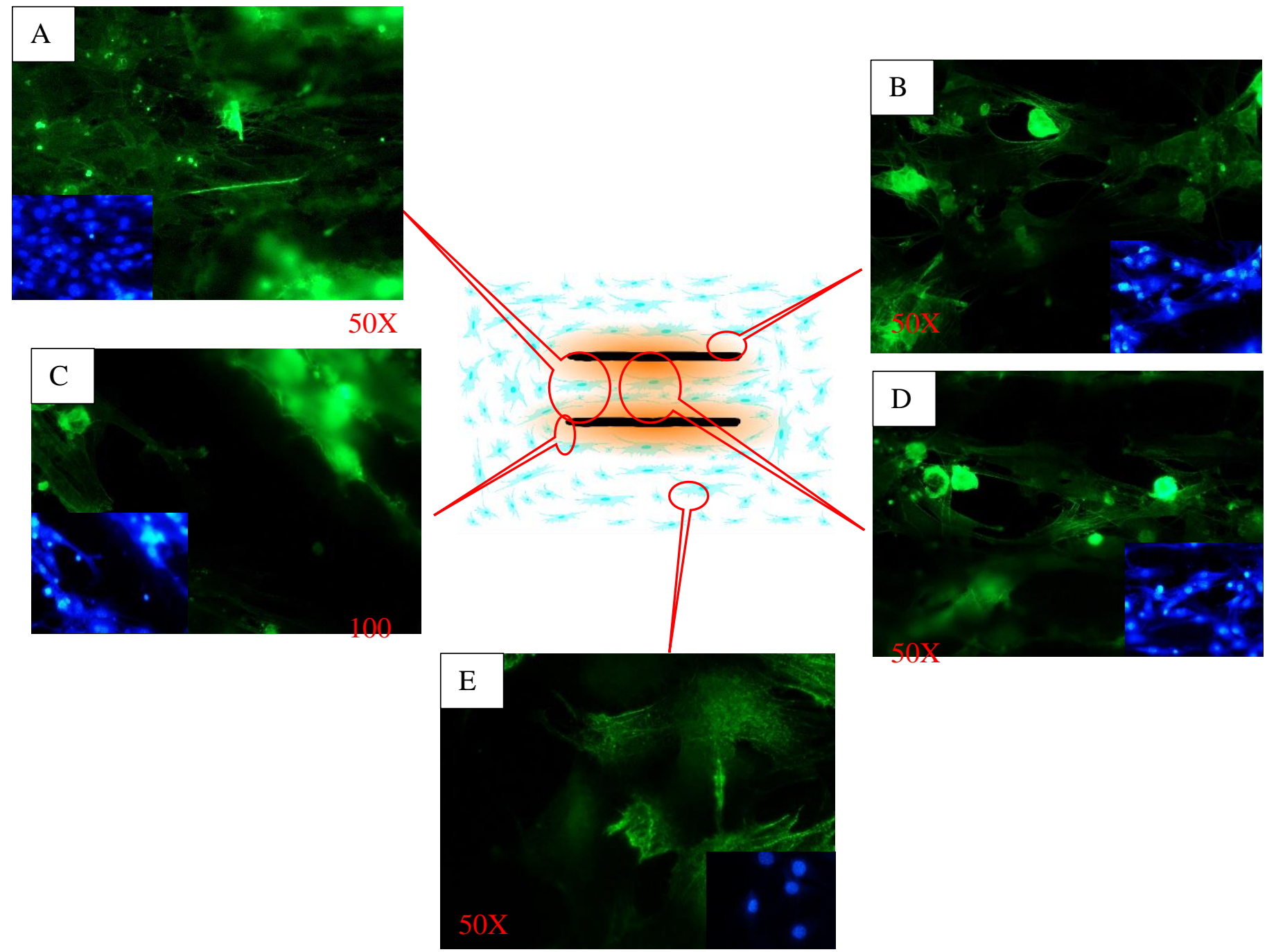

Figure 6-9: Fluorescent microscope data on MC3T3-E1 osteoblast cell channeling, selective cell migration and repellence by nano-printed structure in 24 Hours

The activity of nano printed structure on MC 3T3-E1 osteoblast cells reveal some fascinating areas for selective cell proliferation and channeling applications. Focal adhesion complex is identified as a key ingredient for cell adhesion. Hence, a proliferating cell on figure 6-9 (E) have randomly oriented uniform spread focal adhesions. But, the activity of cells on and near the nano-printed titanium surface is different. Figure 6-9 (A) shows alignment of cells / cell channelling when in contact in-between two printed structure. But, when the MC3T3-E1 osteoblast cells come in contact close to the nano-printed area where the density of the synthesized rutile dominant phase is high an acute change in cell morphology is observed from figure 6-9 (B,C). This change in cell morphology is accounted as cell repellence. Then, evidence of cell elongation is also observed on the nano-printed structure in figure 6-9 (E) where the proliferated cell seem to have stretched nucleus when comparing with the proliferated cell on the native control surface figure 6-9 (A). 
Thus, the nano-printed is acting as a smart interactive structure allowing both cell proliferation and cell repellence on the same nano-printed structure.

\subsection{Influence of synthesized patterns on fibroblast cell}
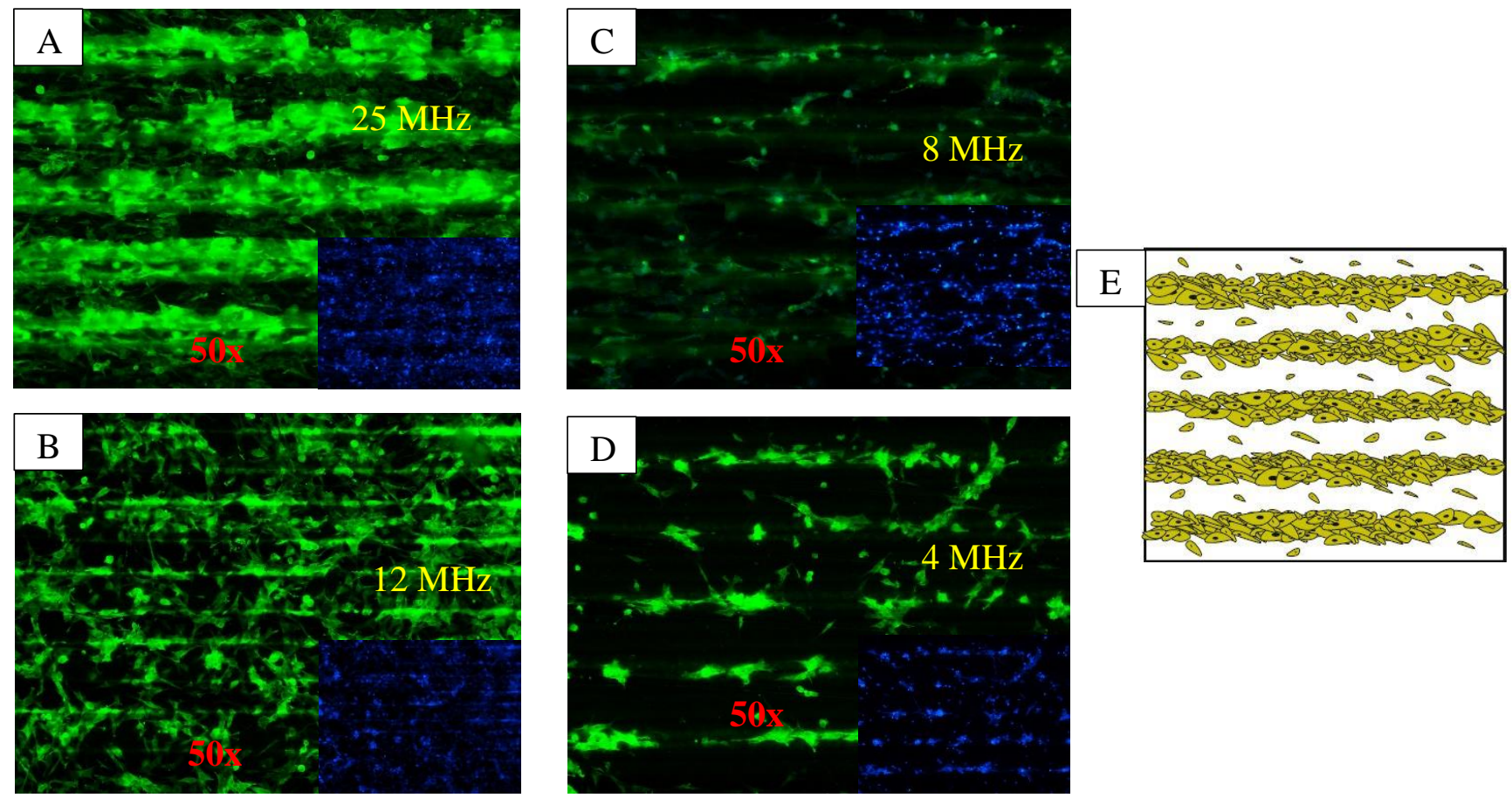

Figure 6-10: Fluorescent microscopic image on effect of NIH3T3 fibroblast cell proliferation on each synthesized pattern (A-D) after 24 Hour incubation. (E)- Illustration of pattern synthesized with preferential cell attachment only on laser irradiated surface

The above figure 6-10 (A-D) evidently show a trend with gradual reduction in cell density/ proliferation from figure 6-10 (A) to (D) due to increased laser peak power and degree of phase transformation. Thus the above synthesized laser irradiated surface pattern generated a similar cell response from NIH3T3 fibroblast thereby further verifying the elucidated response from MC3T3E1 osteoblast figure 6-(6,7) was from synthesized surface patterns. 


\subsection{Influence of selective nano printing on fibroblast cell}
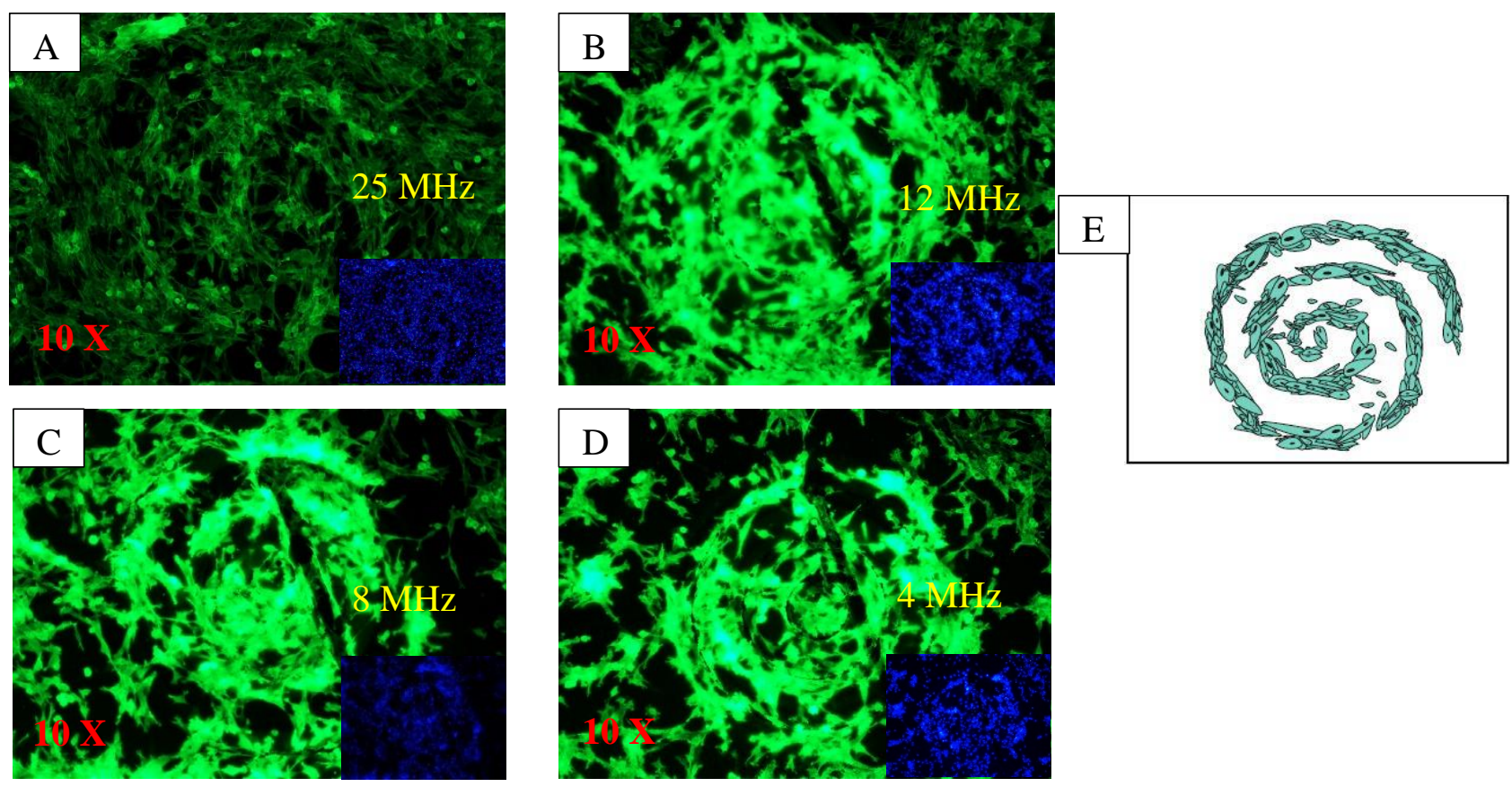

Figure 6-11: Fluorescent microscopic image on effect of NIH3T3 fibroblast cell proliferation on each nano printed pattern (A-D) after 24 Hour incubation. (E)- Illustration of nano printed pattern by USP laser having preferential cell attachment only on laser printed surface

The above figure 6-11 illustrates NIH3T3 fibroblast cell activity on laser nano-printed pattern where preferential cell proliferation was evidenced on the printed area. A uniform alignment of cells over the printed area was seen in figure 6-11 (A) due to low laser peak power irradiation and reduction in cell proliferation and alignment was witnessed in figure 6-11 (D) due to high laser peak power irradiation condition. The cytoskeletal information also reveal cell clustering on the nano-printed areas figure 6-11 (B-D) with gradual reduction in both cell clustering and cell proliferation near the printed region. 


\subsection{Summary}

We have conducted a yet another way to selectively functionalize a titanium by both surface phase transformation patterning and printing nanostructures over native titanium surface by USP laser. The surface pattern synthesized surface was dominated by rutile phase oxide which contributed to specific orientation and aligning of osteoblast and fibroblast cells with reduced proliferation activity. The nano structures printed surface showcased a similar effect on rutile phase dominant structure with added influence of cell repellence due to high density and smaller nanoparticles synthesized in the nanostructure. 


\section{Conclusion}

In this dissertation, a diverse range of engineering concepts was investigated which included: lasermaterial interaction, oxide phase transformations, material characterization and tissue engineering. The main objective of this dissertation was to introduce a new synthesizing technique for titanium based biomaterial using USP laser in ambient conditions. Later the results from ablation of titanium targets indicated that USP laser ablation in air yielded both crystalline oxide phase surface and nanostructures.

The influence of synthesized biomaterial were tested in vitro proliferation and viability of osteoblast-like MC3T3-E1 cells and NIH 3T3 mouse embryonic fibroblasts. The results also indicated that the surface morphology as well as surface physiochemical properties of the synthesized biomaterial significantly influenced cell controllability by inducing selective cell apoptosis due to rutile phase dominance nanostructure and directional cell migration in the phase dominant surface. The cell count numbers on the synthesized substrates and nanostructures changed consistently with oxide phase dominance and density of synthesized nanofibrous structures. Cell cytoskeleton and nucleus studies revealed dramatic size reduction in nucleus on synthesized surface and nanostructure.

\subsection{Recommended future work}

Although, good progress was made on studying the engineered properties of new biomaterial synthesized by USP laser, there are many unanswered questions and exciting avenues of research to explore with USP laser. These avenues cross many fields including tissue engineering, biosensors and more. Further research on double pulse laser ablation of titanium can be done at various laser pulse wavelengths in order to predict the new phases synthesized due to shorter laser material interaction time and study their interaction with primary cells lines. Also, ablation of titanium can be performed at different background gas to investigate the influence of ambient gas on phase transformation and the synthesized nanostructures. Then, the effect of these synthesized nanostructures can be evaluated to other mammalian and cancer cell lines. 


\section{Appendix - A1}

\begin{tabular}{|r|r|r|r|r|}
\hline Average Power & Pulse Rates & Pulse Widths & Pulse energy & Peak power \\
\hline Watts & Mega Hertz & Femtoseconds & micro joules & watts \\
\hline 16 & 25.07 & 214 & $6.38213 \mathrm{E}-07$ & 2982303.755 \\
\hline 16 & 12.5 & 214 & 0.00000128 & 5981308.411 \\
\hline 16 & 8.36 & 214 & $1.91388 \mathrm{E}-06$ & 8943343.916 \\
\hline 16 & 4.18 & 214 & $3.82775 \mathrm{E}-06$ & 17886687.83 \\
\hline 16 & 25.07 & 714 & $6.38213 \mathrm{E}-07$ & 893855.7473 \\
\hline 16 & 12.5 & 714 & 0.00000128 & 1792717.087 \\
\hline 16 & 8.36 & 714 & $1.91388 \mathrm{E}-06$ & 2680498.037 \\
\hline 16 & 4.18 & 714 & $3.82775 \mathrm{E}-06$ & 5360996.073 \\
\hline 16 & 25.07 & 1428 & $6.38213 \mathrm{E}-07$ & 446927.8737 \\
\hline 16 & 12.5 & 1428 & 0.00000128 & 896358.5434 \\
\hline 16 & 8.36 & 1428 & $1.91388 \mathrm{E}-06$ & 1340249.018 \\
\hline 16 & 4.18 & 1428 & $3.82775 \mathrm{E}-06$ & 2680498.037 \\
\hline
\end{tabular}




\section{References}

1. Bauer, Sebastian, Patrik Schmuki, Klaus von der Mark, and Jung Park. "Engineering Biocompatible Implant Surfaces: Part I: Materials and Surfaces." Progress in Materials Science 58, no. 3 (April 2013): 261-326. doi:10.1016/j.pmatsci.2012.09.001.

2. Matschegewski, Claudia, Susanne Staehlke, Ronny Loeffler, Regina Lange, Feng Chai, Dieter P. Kern, Ulrich Beck, and Barbara J. Nebe. "Cell Architecture-cell Function Dependencies on Titanium Arrays with Regular Geometry." Biomaterials 31, no. 22 (August 2010): 5729-40. doi:10.1016/j.biomaterials.2010.03.073.

3. Lüthen, Frank, Regina Lange, Petra Becker, Joachim Rychly, Ulrich Beck, and J. G. Barbara Nebe. "The Influence of Surface Roughness of Titanium on $\beta 1$ - and $\beta 3$-Integrin Adhesion and the Organization of Fibronectin in Human Osteoblastic Cells.” Biomaterials 26, no. 15 (May 2005): 2423-40. doi:10.1016/j.biomaterials.2004.07.054.

4. Lauer, G, M Wiedmann-Al-Ahmad, J. E Otten, U Hübner, R Schmelzeisen, and W Schilli. "The Titanium Surface Texture Effects Adherence and Growth of Human Gingival Keratinocytes and Human Maxillar Osteoblast-like Cells in Vitro.” Biomaterials 22, no. 20 (October 15, 2001): 2799 2809. doi:10.1016/S0142-9612(01)00024-2.

5. Park, Jung, Sebastian Bauer, Klaus von der Mark, and Patrik Schmuki. "Nanosize and Vitality: TiO2 Nanotube Diameter Directs Cell Fate." Nano Letters 7, no. 6 (June 1, 2007): 1686-91. doi:10.1021/n1070678d.

6. Mendonça, Gustavo, Daniela B. S. Mendonça, Francisco J. L. Aragão, and Lyndon F. Cooper. "Advancing Dental Implant Surface Technology - From Micron- to Nanotopography." Biomaterials 29, no. 28 (October 2008): 3822-35. doi:10.1016/j.biomaterials.2008.05.012.

7. Mendonça, Gustavo, Daniela B. S. Mendonça, Luis G. P. Simões, André L. Araújo, Edson R. Leite, Wagner R. Duarte, Francisco J. L. Aragão, and Lyndon F. Cooper. "The Effects of Implant Surface Nanoscale Features on Osteoblast-Specific Gene Expression." Biomaterials 30, no. 25 (September 2009): 4053-62. doi:10.1016/j.biomaterials.2009.04.010.

8. Zhao, Lingzhou, Shenglin Mei, Paul K. Chu, Yumei Zhang, and Zhifen Wu. "The Influence of Hierarchical Hybrid Micro/nano-Textured Titanium Surface with Titania Nanotubes on Osteoblast Functions." Biomaterials 31, no. 19 (July 2010): 5072-82. doi:10.1016/j.biomaterials.2010.03.014.

9. Wei, Guobao, Qiming Jin, William V. Giannobile, and Peter X. Ma. "The Enhancement of Osteogenesis by Nano-Fibrous Scaffolds Incorporating rhBMP-7 Nanospheres." Biomaterials 28, no. 12 (April 2007): 2087-96. doi:10.1016/j.biomaterials.2006.12.028. 
10. Liu, Xiaohua, Laura A. Smith, Jiang Hu, and Peter X. Ma. "Biomimetic Nanofibrous Gelatin/apatite Composite Scaffolds for Bone Tissue Engineering." Biomaterials 30, no. 12 (April 2009): 225258. doi:10.1016/j.biomaterials.2008.12.068.

11. Geetha, M., A. K. Singh, R. Asokamani, and A. K. Gogia. "Ti Based Biomaterials, the Ultimate Choice for Orthopaedic Implants - A Review." Progress in Materials Science 54, no. 3 (May 2009): 397-425. doi:10.1016/j.pmatsci.2008.06.004.

12. Divya Rani, V V, K Manzoor, Deepthy Menon, N Selvamurugan, and Shantikumar V Nair. "The Design of Novel Nanostructures on Titanium by Solution Chemistry for an Improved Osteoblast Response.” Nanotechnology 20, no. 19 (May 13, 2009): 195101. doi:10.1088/09574484/20/19/195101.

13. Galli, Carlo, Guido M. Macaluso, Erida Elezi, Francesca Ravanetti, Antonio Cacchioli, Giacomo Gualini, and Giovanni Passeri. "The Effects of Er:YAG Laser Treatment on Titanium Surface Profile and Osteoblastic Cell Activity: An In Vitro Study." Journal of Periodontology 82, no. 8 (December 28, 2010): 1169-77. doi:10.1902/jop.2010.100428.

14. R.Geoff Richards, T.Fintan Moriarty, Theodore Miclau,Robert T.McClellan, David W.Grainger, "Advances in biomaterials and surface technologies", Journal ortho Trauma, Volume 26, no. 12 (December 2012):

15. De Nardo, Luigi, Giuseppina Raffaini, Edward Ebramzadeh, and Fabio Ganazzoli. "Titanium Oxide Modeling and Design for Innovative Biomedical Surfaces: A Concise Review." The International Journal of Artificial Organs 35, no. 9 (2012): 629-41. doi:10.5301/ijao.5000040.

16. Das, Kakoli, Susmita Bose, and Amit Bandyopadhyay. "Surface Modifications and Cell-materials Interactions with Anodized Ti." Acta Biomaterialia 3, no. 4 (July 2007): 573-85. doi:10.1016/j.actbio.2006.12.003.

17. Gutwein, Luke G., and Thomas J. Webster. "Increased Viable Osteoblast Density in the Presence of Nanophase Compared to Conventional Alumina and Titania Particles." Biomaterials 25, no. 18 (August 2004): 4175-83. doi:10.1016/j.biomaterials.2003.10.090.

18. Zieliński, A., Sylwia Sobieszczyk, Waldemar Serbiński, Tomasz Seramak, and A. Ossowska. "Materials Design for the Titanium Scaffold Based Implant." Solid State Phenomena 183 (2012): $225-32$. 
19. Carbone, Roberta, Ida Marangi, Andrea Zanardi, Luca Giorgetti, Elisabetta Chierici, Giuseppe Berlanda, Alessandro Podestà, et al. "Biocompatibility of Cluster-Assembled Nanostructured TiO2 with Primary and Cancer Cells." Biomaterials 27, no. 17 (June 2006): 3221-29. doi:10.1016/j.biomaterials.2006.01.056.

20. Lin, Jinyou, Xianfeng Wang, Bin Ding, Jianyong Yu, Gang Sun, and Moran Wang. "Biomimicry via Electrospinning." Critical Reviews in Solid State and Materials Sciences 37, no. 2 (2012): 94114.

21. Li, Dan, and Younan Xia. "Electrospinning of Nanofibers: Reinventing the Wheel?" Advanced Materials 16, no. 14 (2004): 1151-70.

22. Zhang, Chuan-Ling, and Shu-Hong Yu. "Nanoparticles Meet Electrospinning: Recent Advances and Future Prospects." Chemical Society Reviews, 2014.

23. Lin, Jinyou, Bin Ding, and Jianyong Yu. "Direct Fabrication of Highly Nanoporous Polystyrene Fibers via Electrospinning." ACS Applied Materials \& Interfaces 2, no. 2 (2010): 521-28.

24. Zhao, Yong, Xinyu Cao, and Lei Jiang. "Bio-Mimic Multichannel Microtubes by a Facile Method." Journal of the American Chemical Society 129, no. 4 (2007): 764-65.

25. McCann, Jesse T., Manuel Marquez, and Younan Xia. "Highly Porous Fibers by Electrospinning into a Cryogenic Liquid." Journal of the American Chemical Society 128, no. 5 (2006): 1436-37.

26. Frandsen, Christine J., Karla S. Brammer, and Sungho Jin. "Variations to the Nanotube Surface for Bone Regeneration." International Journal of Biomaterials 2013 (2013).

27. Fahim, Narges F., and Tohru Sekino. "A Novel Method for Synthesis of Titania Nanotube Powders Using Rapid Breakdown Anodization." Chemistry of Materials 21, no. 9 (2009): 1967-79.

28. Rani, Sanju, Somnath C. Roy, Maggie Paulose, Oomman K. Varghese, Gopal K. Mor, Sanghoon Kim, Sorachon Yoriya, Thomas J. LaTempa, and C. A. Grimes. "Synthesis and Applications of Electrochemically Self-Assembled Titania Nanotube Arrays." Physical Chemistry Chemical Physics 12, no. 12 (2010): 2780-2800.

29. P. Enique, Handbook of growth factors, Boca Raton: CRC Press, 1994

30. Von der Mark, Klaus, and Jung Park. "Engineering Biocompatible Implant Surfaces: Part II: Cellular Recognition of Biomaterial Surfaces: Lessons from Cell-matrix Interactions." Progress in Materials Science 58, no. 3 (April 2013): 327-81. doi:10.1016/j.pmatsci.2012.09.002. 
31. Xiao, Peng, Betzaida Batalla Garcia, Qing Guo, Dawei Liu, and Guozhong Cao. "TiO 2 Nanotube Arrays Fabricated by Anodization in Different Electrolytes for Biosensing." Electrochemistry Communications 9, no. 9 (2007): 2441-47.

32. Paulose, Maggie, Karthik Shankar, Oomman K. Varghese, Gopal K. Mor, Brian Hardin, and Craig A. Grimes. "Backside Illuminated Dye-Sensitized Solar Cells Based on Titania Nanotube Array Electrodes.” Nanotechnology 17, no. 5 (2006): 1446.

33. Barnes, Catherine P., Scott A. Sell, Eugene D. Boland, David G. Simpson, and Gary L. Bowlin. "Nanofiber Technology: Designing the next Generation of Tissue Engineering Scaffolds." Advanced Drug Delivery Reviews 59, no. 14 (2007): 1413-33.

34. Vasilev, Krasimir, Zihan Poh, Krishna Kant, Joseph Chan, Andrew Michelmore, and Dusan Losic. "Tailoring the Surface Functionalities of Titania Nanotube Arrays." Biomaterials 31, no. 3 (2010): 532-40.

35. Tavangar, Amirhossein, Bo Tan, and K. Venkatakrishnan. "Single-Step Fabrication of Microfluidic Channels Filled with Nanofibrous Membrane Using Femtosecond Laser Irradiation." Journal of Micromechanics and Microengineering 20, no. 8 (2010): 085016.

36. Pedrotti FL, Pedrotti LS : Introduction to Optics , New jersey :Prentice hall;1983

37. Gamaly, E. G. "The Physics of Ultra-Short Laser Interaction with Solids at Non-Relativistic Intensities." Physics Reports 508, no. 4-5 (November 2011): 91-243. doi:10.1016/j.physrep.2011.07.002.

38. Tavangar, Amirhossein, Bo Tan, and K. Venkatakrishnan. "Study of the Formation of 3-D Titania Nanofibrous Structure by MHz Femtosecond Laser in Ambient Air." Journal of Applied Physics 113, no. 2 (January 2013): 023102-023102-9. doi:10.1063/1.4771667.

39. Tavangar, Amirhossein, Bo Tan, and K. Venkatakrishnan. "Synthesis of Bio-Functionalized ThreeDimensional Titania Nanofibrous Structures Using Femtosecond Laser Ablation." Acta Biomaterialia 7, no. 6 (2011): 2726-32.

40. Maznichenko, Dmitry, Krishnan Venkatakrishnan, and Bo Tan. "Stimulating Multiple SERS Mechanisms by a Nanofibrous Three-Dimensional Network Structure of Titanium Dioxide (TiO2)." The Journal of Physical Chemistry C 117, no. 1 (2012): 578-83.

41. Perrière, Jacques, Chantal Boulmer-Leborgne, Ratiba Benzerga, and Sylvain Tricot. "Nanoparticle Formation by Femtosecond Laser Ablation.” Journal of Physics D: Applied Physics 40, no. 22 (2007): 7069. 
42. Ullmann, Marc, Sheldon K. Friedlander, and Andreas Schmidt-Ott. "Nanoparticle Formation by Laser Ablation." Journal of Nanoparticle Research 4, no. 6 (2002): 499-509.

43. Chen, Xiao-Bo, Yun-Cang Li, Peter D. Hodgson, and Cuie Wen. "The Importance of Particle Size in Porous Titanium and Nonporous Counterparts for Surface Energy and Its Impact on Apatite Formation." Acta Biomaterialia 5, no. 6 (2009): 2290-2302.

44. Sivayoganathan, Mugunthan, Bo Tan, and Krishnan Venkatakrishnan. "Formation of RingPatterned Nanoclusters by Laser-plume Interaction." Journal of Nanoparticle Research 15, no. 1 (2013): 1-9.

45. Ting, Chu-Chi, San-Yuan Chen, and Dean-Mo Liu. "Structural Evolution and Optical Properties of TiO 2 Thin Films Prepared by Thermal Oxidation of Sputtered Ti Films." Journal of Applied Physics 88, no. 8 (2000): 4628-33.

46. Rasti, Negar, Ehsan Toyserkani, and Fathy Ismail. "Chemical Modification of Titanium Immersed in Hydrogen Peroxide Using Nanosecond Pulsed Fiber Laser Irradiation.” Materials Letters 65, no. 6 (2011): 951-54.

47. Growth, Jour Cryst. "Rigaku Publication List (Thin Film)." Rigaku Publication List-TF 28/Dec/2013 113 (2013): 83.

48. Semaltianos, N. G., S. Logothetidis, N. Frangis, I. Tsiaoussis, W. Perrie, G. Dearden, and K. G. Watkins. "Laser Ablation in Water: A Route to Synthesize Nanoparticles of Titanium Monoxide." Chemical Physics Letters 496, no. 1 (2010): 113-16.

49. Pérez del Pino, A., P. Serra, and J. L. Morenza. "Coloring of Titanium by Pulsed Laser Processing in Air.” Thin Solid Films 415, no. 1 (2002): 201-5.

50. Miura, Shingo, and Jun Takebe. "Biological Behavior of Fibroblast-like Cells Cultured on Anodized-Hydrothermally Treated Titanium with a Nanotopographic Surface Structure." Journal of Prosthodontic Research 56, no. 3 (2012): 178-86.

51. Ward, Brian C., and Thomas J. Webster. "Increased Functions of Osteoblasts on Nanophase Metals." Materials Science and Engineering: C 27, no. 3 (2007): 575-78.

52. Chen, J., J. P. Ulerich, E. Abelev, A. Fasasi, C. B. Arnold, and W. O. Soboyejo. "An Investigation of the Initial Attachment and Orientation of Osteoblast-like Cells on Laser Grooved Ti-6Al-4V Surfaces." Materials Science and Engineering: C 29, no. 4 (2009): 1442-52. 
53. Curtis, A. S. G., N. Gadegaard, M. J. Dalby, M. O. Riehle, C. D. W. Wilkinson, and G. Aitchison. "Cells React to Nanoscale Order and Symmetry in Their Surroundings." NanoBioscience, IEEE Transactions on 3, no. 1 (2004): 61-65.

54. Soboyejo, W. O., B. Nemetski, S. Allameh, N. Marcantonio, C. Mercer, and J. Ricci. "Interactions between MC3T3-E1 Cells and Textured Ti6A14V Surfaces." Journal of Biomedical Materials Research 62, no. 1 (2002): 56-72.

55. Chen, J., S. Mwenifumbo, C. Langhammer, J.-P. McGovern, M. Li, A. Beye, and W. O. Soboyejo. "Cell/surface Interactions and Adhesion on Ti-6Al-4V: Effects of Surface Texture." Journal of Biomedical Materials Research Part B: Applied Biomaterials 82, no. 2 (2007): 360-73.

56. Webster, Thomas J., and Jeremiah U. Ejiofor. "Increased Osteoblast Adhesion on Nanophase Metals: Ti, Ti6Al4V, and CoCrMo.” Biomaterials 25, no. 19 (2004): 4731-39.

57. Dinan, B., D. Gallego-Perez, H. Lee, D. Hansford, and S. A. Akbar. "Thermally Grown $\mathrm{TiO}_{2}$ Nanowires to Improve Cell Growth and Proliferation on Titanium Based Materials." Ceramics International 39, no. 5 (2013): 5949-54.

58. Bleiner, Davide. "Mathematical Modelling of Laser-Induced Particulate Formation in Direct Solid Microanalysis." Spectrochimica Acta Part B: Atomic Spectroscopy 60, no. 1 (2005): 49-64.

59. Kiani, Amirkianoosh, Krishnan Venkatakrishnan, Bo Tan, and Venkat Venkataramanan. "Maskless Lithography Using Silicon Oxide Etch-Stop Layer Induced by Megahertz Repetition Femtosecond Laser Pulses." Optics Express 19, no. 11 (2011): 10834-42.

60. Brammer, Karla S., Seunghan Oh, John O. Gallagher, and Sungho Jin. "Enhanced Cellular Mobility Guided by TiO2 Nanotube Surfaces." Nano Letters 8, no. 3 (2008): 786-93.

61. Park, Hyoungshin, Christopher Cannizzaro, Gordana Vunjak-Novakovic, Robert Langer, Charles A. Vacanti, and Omid C. Farokhzad. "Nanofabrication and Microfabrication of Functional Materials for Tissue Engineering." Tissue Engineering 13, no. 8 (2007): 1867-77.

62. Ainslie, Kristy M., and Tejal A. Desai. "Microfabricated Implants for Applications in Therapeutic Delivery, Tissue Engineering, and Biosensing." Lab on a Chip 8, no. 11 (2008): 1864-78.

63. Ma, Zuwei, Wei He, Thomas Yong, and S. Ramakrishna. "Grafting of Gelatin on Electrospun Poly (caprolactone) Nanofibers to Improve Endothelial Cell Spreading and Proliferation and to Control Cell Orientation.” Tissue Engineering 11, no. 7-8 (2005): 1149-58.

64. Dalby, Matthew J. "Cellular Response to Low Adhesion Nanotopographies." International Journal of Nanomedicine 2, no. 3 (2007): 373. 
65. Dalby, Matthew John, Mathis O. Riehle, Duncan S. Sutherland, Hossein Agheli, and Adam SG Curtis. "Changes in Fibroblast Morphology in Response to Nano-Columns Produced by Colloidal Lithography.” Biomaterials 25, no. 23 (2004): 5415-22.

66. Dalby, M. J., S. Childs, M. O. Riehle, H. J. H. Johnstone, S. Affrossman, and A. S. G. Curtis. "Fibroblast Reaction to Island Topography: Changes in Cytoskeleton and Morphology with Time." Biomaterials 24, no. 6 (2003): 927-35.

67. Dalby, Matthew J., Nikolaj Gadegaard, Pawel Herzyk, Hossein Agheli, Duncan S. Sutherland, and Chris DW Wilkinson. "Group Analysis of Regulation of Fibroblast Genome on Low-Adhesion Nanostructures.” Biomaterials 28, no. 10 (2007): 1761-69.

68. Rodriguez, Laura Lara, and Ian C. Schneider. "Directed Cell Migration in Multi-Cue Environments." Integr. Biol. 5, no. 11 (2013): 1306-23.

69. Friedl, Peter, and Katarina Wolf. "Plasticity of Cell Migration: A Multiscale Tuning Model." The Journal of Cell Biology 188, no. 1 (2010): 11-19.

70. Phillipson, Mia, and Paul Kubes. "The Neutrophil in Vascular Inflammation.” Nature Medicine 17, no. 11 (2011): 1381-90.

71. Prasad, Mohit, and Denise J. Montell. "Cellular and Molecular Mechanisms of Border Cell Migration Analyzed Using Time-Lapse Live-Cell Imaging." Developmental Cell 12, no. 6 (2007): 997-1005.

72. Arora, S., J. Jain, J. M. Rajwade, and K. M. Paknikar. "Cellular Responses Induced by Silver Nanoparticles:In Vitro Studies.” Toxicology Letters 179, no. 2 (2008): 93-100.

73. Sjöström, Terje, Laura E. McNamara, Li Yang, Matthew J. Dalby, and Bo Su. "Novel Anodization Technique Using a Block Copolymer Template for Nanopatterning of Titanium Implant Surfaces." ACS Applied Materials \& Interfaces 4, no. 11 (2012): 6354-61.

74. Falconnet, Didier, Gabor Csucs, H. Michelle Grandin, and Marcus Textor. "Surface Engineering Approaches to Micropattern Surfaces for Cell-Based Assays." Biomaterials 27, no. 16 (2006): 3044-63.

75. Chen, Christopher S., Milan Mrksich, Sui Huang, George M. Whitesides, and Donald E. Ingber. "Geometric Control of Cell Life and Death." Science 276, no. 5317 (1997): 1425-28. 
76. McBeath, Rowena, Dana M. Pirone, Celeste M. Nelson, Kiran Bhadriraju, and Christopher S. Chen. "Cell Shape, Cytoskeletal Tension, and RhoA Regulate Stem Cell Lineage Commitment." Developmental Cell 6, no. 4 (2004): 483-95.

77. Lord, Megan Susan, Charlotte Modin, Morten Foss, Mogens Duch, Anne Simmons, Finn S. Pedersen, Bruce K. Milthorpe, and Flemming Besenbacher. "Monitoring Cell Adhesion on Tantalum and Oxidised Polystyrene Using a Quartz Crystal Microbalance with Dissipation." Biomaterials 27, no. 26 (2006): 4529-37.

78. Zinger, O., G. Zhao, Z. Schwartz, J. Simpson, M. Wieland, D. Landolt, and Barbara Boyan. "Differential Regulation of Osteoblasts by Substrate Microstructural Features." Biomaterials 26, no. 14 (2005): 1837-47.

79. Flemming, R. G., C. J. Murphy, G. A. Abrams, S. L. Goodman, and P. F. Nealey. "Effects of Synthetic Micro-and Nano-Structured Surfaces on Cell Behavior." Biomaterials 20, no. 6 (1999): 573-88. 